JOURNAL OF THE

AMERICAN MATHEMATICAL SOCIETY

Volume 17, Number 3, Pages 499-555

S 0894-0347(04)00458-8

Article electronically published on April 26, 2004

\title{
INTEGRAL MOTIVES AND SPECIAL VALUES OF ZETA FUNCTIONS
}

\author{
JAMES S. MILNE AND NIRANJAN RAMACHANDRAN
}

\section{Contents}

Introduction 499

1. The realization categories $\quad 502$

2. Adèlic cohomology $\quad 506$

3. An adèlic Tate conjecture $\quad 508$

4. The adèlic fibre functor $\quad 510$

5. Categories of (integral) motives 513

6. Tannakian properties

7. Some spectral sequences

8. Homological algebra in the category of motives 534

9. Extensions and zeta functions: Local case 539

10. Extensions and zeta functions: Global case 546

Acknowledgements $\quad 553$

References 553

\section{INTRODUCTION}

For each field $k$, there is conjecturally a category of mixed isomotives whose full subcategory of semisimple objects is the category $\mathcal{M}(k ; \mathbb{Q})$ of pure isomotives. In this paper, we define a $\mathbb{Z}$-category $\mathcal{M}(k ; \mathbb{Z})$ of motives whose quotient by its subcategory of torsion objects is $\mathcal{M}(k ; \mathbb{Q})$. Thus, $\mathcal{M}(k ; \mathbb{Z})$ is the abelian category of mixed motives over $k$ whose weight filtrations split modulo torsion. As Grothendieck observed, when $k$ is finite, the weight filtrations always split modulo torsion, and so, in this case, $\mathcal{M}(k ; \mathbb{Z})$ is the full category of mixed motives over $k$.

The category $\mathcal{M}(k ; \mathbb{Z})$ depends on our choice of $\mathcal{M}(k ; \mathbb{Q})$. For definiteness, take $\mathcal{M}(k ; \mathbb{Q})$ to be the category of isomotives based on the smooth projective varieties over $k$ whose Künneth projectors are algebraic - the correspondences are the numerical equivalence classes of algebraic classes. This category is semisimple [26] and Tannakian [8], and when we assume that numerical equivalence coincides with

Received by the editors June 11, 2002.

2000 Mathematics Subject Classification. Primary 11G09; Secondary 14F42, 14G10.

The first author received support from the National Science Foundation and the second author from MPIM (Bonn) and a GRB Summer Grant (UMD). 
homological equivalence, it admits canonical $l$-adic fibre functors for each $l$, The category $\mathcal{M}(k ; \mathbb{Z})$ that we construct is noetherian, abelian, and, at least when $k$ is finite and the Tate conjecture holds, Tannakian.

The Ext groups in the full category $\mathcal{M}(k ; \mathbb{Z})$ seem to be pathological (cf. 9.7), but those in the subcategory $\mathcal{M}^{+}(k ; \mathbb{Z})$ of effective motives are of interest. When $k$ is finite, we show that the Tate conjecture implies relations between these Ext groups and the behaviour of zeta functions near integers (Theorems 10.1 10.5). Moreover, to a smooth projective variety $V$ satisfying a certain degeneration condition on its crystalline cohomology, we are able to attach "Weil" motivic cohomology groups $H_{\text {mot }}^{i}(V, \mathbb{Z}(r))$ satisfying the spectral sequence

$$
H^{i}\left(\Gamma_{0}, H_{\mathrm{mot}}^{j}(\bar{V}, \mathbb{Z}(r)) \Longrightarrow H_{\mathrm{mot}}^{i+j}(V, \mathbb{Z}(r))\right.
$$

posited by Lichtenbaum [35], and deduce from Theorem 10.5 that

$$
\zeta(V, s) \sim \pm \chi^{\times}(V, \mathbb{Z}(r)) \cdot q^{\chi\left(V, \mathcal{O}_{V}, r\right)} \cdot\left(1-q^{r-s}\right)^{\rho} \text { as } s \rightarrow r
$$

(Theorem 10.7). Here $\Gamma_{0}$ is the subgroup of $\operatorname{Gal}\left(\mathbb{F} / \mathbb{F}_{q}\right)$ generated by the Frobenius element (so $\left.\Gamma_{0} \cong \mathbb{Z}\right), \chi^{\times}(V, \mathbb{Z}(r))$ is the alternating product of the orders of the cohomology groups of a complex

$$
\cdots \rightarrow H_{\mathrm{mot}}^{i}(V, \mathbb{Z}(r)) \rightarrow H_{\mathrm{mot}}^{i+1}(V, \mathbb{Z}(r)) \rightarrow \cdots
$$

arising from (1), $\chi\left(V, \mathcal{O}_{V}, r\right)$ is as in [38, and $\rho=\sum(-1)^{i} i \cdot \operatorname{rank} H_{\mathrm{mot}}^{i}(V, \mathbb{Z}(r))$.

The category $\mathcal{M}(k ; \mathbb{Z})$ is defined in $\S 5$ as a full subcategory of the fibre product of the $\mathbb{Q}$-category $\mathcal{M}(k ; \mathbb{Q})$ with a certain $\hat{\mathbb{Z}}$-category of realizations. The construction requires that we show that the various $l$-adic fibre functors on $\mathcal{M}(k ; \mathbb{Q})$ define an adèlic fibre functor - the proof of this depends crucially on the theorem of Gabber 17]. In $\S 4$, we show that the usual Tate conjecture implies an adèlic version. For example, for a variety over a finite field, if the $l$-adic Tate conjecture holds for a single $l$, then it holds $l$-integrally for almost all $l$.

The category $\mathcal{M}\left(\mathbb{F}_{q} ; \mathbb{Z}\right)$ is shown to be Tannakian in $\S 6$, and the main results on Exts and motivic cohomology groups are obtained in $\S 10$ after preliminaries in $\S \S 7-9$.

This article is part of a project whose ultimate goal is to define a triangulated category of motives with $t$-structure whose heart is the category $\mathcal{M}(k ; \mathbb{Z})$ defined above, and to show that the Tate conjecture for smooth projective varieties implies the formula (2) for arbitrary varieties over $\mathbb{F}_{q}$.

We note that our approach to motives is opposite to that of other authors ([21], [22], 34], [57]) who define a triangulated category of motives with the goal of putting a $t$-structure on it whose heart will be the abelian category of motives. While our approach can be expected to give the full category of mixed motives only when the ground field is $\mathbb{F}_{q}$ or $\mathbb{F}$, we do expect to obtain the correct $p$-structure, as illustrated by (2). Most of the difficulties in the present work involve the $p$-part in characteristic $p$.

Notations. Throughout, $p$ denotes the characteristic exponent of the ground field (thus, $p$ is the characteristic of the ground field when this is nonzero, and is 1 otherwise), $l$ denotes a prime number, and $\ell$ denotes a prime number $\neq p$. Note

\footnotetext{
${ }^{1}$ For an alternative choice for $\mathcal{M}(k ; \mathbb{Q})$ that does not require any unproven conjectures, and which even satisfies the Tate conjecture, see 5.27 .
} 
that we allow $l=p$, and that there is no restriction on $\ell$ when the ground field has characteristic zero.

The $l$-adic valuations $|\quad|_{l}$ and $\operatorname{ord}_{l}$ are normalized so that $|l|_{l}=l^{-1}$ and $\operatorname{ord}_{l}(l)=$ 1. The expression "for almost all $l$ " means "for all but (possibly) a finite number of $l "$.

The profinite completion of $\mathbb{Z}$ is denoted $\hat{\mathbb{Z}}$ and $\mathbb{A}_{f}=\mathbb{Q} \otimes \hat{\mathbb{Z}}$. Thus, $\hat{\mathbb{Z}}=\prod \mathbb{Z}_{l}$, and we let $\mathbb{Z}^{p}=\prod_{l \neq p} \mathbb{Z}_{l}$ and $\mathbb{A}_{f}^{p}=\mathbb{Q} \otimes \mathbb{Z}^{p}$. When $p=1, \mathbb{Z}^{p}=\hat{\mathbb{Z}}$ and $\mathbb{A}_{f}^{p}=\mathbb{A}_{f}$.

We use $M_{m}$ and $M^{(m)}$, respectively, to denote the kernel and cokernel of multiplication by $m$ on $M$. For a prime $l, M(l)=\bigcup M_{l^{n}}$ is the $l$-primary component of $M$, and $M_{\text {tors }}=\bigcup M_{m}$ is the torsion subobject.

For a module $M$ endowed with an action of a group $\Gamma, M^{\Gamma}$ (resp. $M_{\Gamma}$ ) is the largest submodule (resp. quotient module) of a $\Gamma$-module $M$ on which $\Gamma$ acts trivially. For a module $M$ over a subgroup $U$ of $\Gamma$, the induced module $\operatorname{Ind}_{U}^{\Gamma}(M)$ is defined to be the group of maps $\varphi: \Gamma \rightarrow M$ such that $\varphi(u x)=u \varphi(x)$ for $u \in U$, $x \in \Gamma$. The group $\Gamma$ acts on $\operatorname{Ind}_{U}^{\Gamma}(M)$ by $(g \varphi)(x)=\varphi(x g)$. When $\Gamma$ and $M$ have topologies (for example, the discrete topology for $M$ ), the maps $\varphi$ are required to be continuous.

Lattice always means full lattice: when $R$ is a ring and $V$ is a free $\mathbb{Q} \otimes R$-module of finite rank, an $R$-lattice in $V$ is a finitely generated $R$-submodule $\Lambda$ of $V$ such that the inclusion $\Lambda \hookrightarrow V$ induces an isomorphism $\mathbb{Q} \otimes \Lambda \rightarrow V$.

For a field $k, k^{\text {al }}$ denotes an algebraic closure of $k, k^{\text {sep }}$ a separable (algebraic) closure of $k$, and $k^{\mathrm{pf}}$ a perfect closure of $k$ (for example, $k^{\mathrm{pf}}=k^{p^{-\infty}} \subset k^{\mathrm{al}}$ ).

For a perfect field $k$ of characteristic $p, W=W(k)$ denotes the ring of Witt vectors with coefficients in $k$, and $B(k)$ denotes the field of fractions of $W(k)$. The unique lift of the automorphism $x \mapsto x^{p}: k \rightarrow k$ to $W(k)$ is denoted $\sigma$, and $A=W[F, \sigma]$ is the skew polynomial ring (polynomials in $F$ with the relation $F \cdot a=\sigma(a) \cdot F, a \in W)$.

Generally, rings are assumed to be commutative. For a $\operatorname{ring} R, \operatorname{Mod}(R)$ is the category of $R$-modules and $\operatorname{Modf}(R)$ the category of finitely presented $R$-modules, and for a field $k, \operatorname{Vec}(k)$ is the category of finite-dimensional $k$-vector spaces. When $S$ is an $R$-algebra, we usually abbreviate $M \otimes_{R} S$ to $M_{S}$, and for an $R$-linear category (alias, $R$-category) $\mathcal{M}$, we use $\mathcal{M}_{S}$ to denote the category with the same objects as $\mathcal{M}$, but with $\operatorname{Hom}_{\mathcal{M}_{S}}(X, Y)=\operatorname{Hom}_{\mathcal{M}}(X, Y)_{S}$.

For an affine group scheme $G$ over a noetherian ring $R, \operatorname{Rep}(G ; R)$ denotes the category of linear representations of $G$ on finitely generated $R$-modules.

For tensor structures on categories we use the terminology of [10]. Thus a tensor category $\mathcal{C}$ is a category together with a bifunctor $\otimes: \mathcal{C} \times \mathcal{C} \rightarrow \mathcal{C}$ and compatible associativity and commutativity constraints for which there exists an identity object $\mathbb{1}$. When $\mathcal{C}$ is additive and $\operatorname{End}(\mathbb{1})$ is a commutative $\operatorname{ring} R$, we call $(\mathcal{C}, \otimes)$ a tensor category over $R$ or a tensor $R$-category. When $R^{\prime}$ is an $R$-algebra, an $R^{\prime}$ - fibre functor on an abelian tensor $R$-category is a faithful $R$-linear exact tensor functor $\mathcal{C} \rightarrow \operatorname{Mod}\left(R^{\prime}\right)$.

Given functors $F: \mathcal{A} \rightarrow \mathcal{C}$ and $G: \mathcal{B} \rightarrow \mathcal{C}$, the fibre product category $\mathcal{A} \times_{\mathcal{C}} \mathcal{B}$ has as objects the triples $(A, B, \gamma)$ with $A$ an object of $\mathcal{A}, B$ an object of $\mathcal{B}$, and $\gamma: F(A) \rightarrow G(B)$ an isomorphism; the morphisms are the pairs $a: A \rightarrow A^{\prime}$ and $b: B \rightarrow B^{\prime}$ giving rise to a commutative diagram in $\mathcal{C}$.

A variety $V$ is a geometrically reduced separated scheme of finite type over a field. The group of algebraic cycles of codimension $r$ on $V$ is denoted $Z^{r}(V)$, and $Z_{\sim}^{r}(V)$ 
is the quotient of $Z^{r}(V)$ by some adequate relation $\sim$. We let $Z^{*}(V)=\bigoplus_{r} Z^{r}(V)$ and $H^{*}(V, F)=\bigoplus_{i} H^{i}(V, F)$.

An equivalence class containing $x$ is denoted $[x]$. We also use $[S]$ to denote the cardinality of a finite set $S$. For a homomorphism $f: M \rightarrow N$ of abelian groups whose kernel and cokernel are finite, $z(f)$ is defined to be $[\operatorname{Ker}(f)] /[\operatorname{Coker}(f)]$.

Isomorphisms are denoted $\approx$ and canonical isomorphisms $\cong$.

\section{The Realization Categories}

The categories $\mathcal{R}$ away from $p$. Throughout this subsection, $k$ is a field of characteristic exponent $p$, and $R$ is a topological ring, for example, $R=\mathbb{Z}, \mathbb{Z}_{\ell}, \mathbb{Q}_{\ell}$, $\mathbb{Z}^{p}$, or $\mathbb{A}_{f}^{p}$ with its natural topology (discrete for $\mathbb{Z}$ ).

Definition 1.1. (a) For a profinite group $\Gamma, \mathcal{R}(\Gamma ; R)$ is the category of continuous linear representations of $\Gamma$ on finitely presented $R$-modules.

(b) When $k$ is finitely generated over the prime field,

$$
\mathcal{R}(k ; R)=\mathcal{R}(\Gamma ; R), \quad \Gamma=\operatorname{Gal}\left(k^{\mathrm{sep}} / k\right) .
$$

Otherwise,

$$
\mathcal{R}(k ; R)=\underline{\lim } \mathcal{R}\left(k^{\prime} ; R\right)
$$

where $k^{\prime}$ runs over the subfields of $k$ finitely generated over the prime field.

Remark 1.2. In (1.1b), and elsewhere in the paper, we mean the naive direct limit of categories, not the 2-category direct limit. Specifically, let $I$ be a directed set, and let $d: I \rightarrow \mathbf{C A T}$ be a functor. Then $\lim d(i)$ is the category with

$$
\mathrm{ob}\left(\lim _{\longrightarrow} d(i)\right)=\underset{i}{\lim } \operatorname{ob}(d(i)), \quad \operatorname{ar}(\underline{\lim }(d(i))=\underset{i}{\lim }(\operatorname{ar}(d(i))
$$

and the obvious category structure.

In (1.1 b), fix a separable closure $k^{\text {sep }}$ of $k$ and, for $k^{\prime} \subset k$, let $k^{\text {sep }}$ be the separable closure of $k^{\prime}$ in $k^{\text {sep }}$. If $k^{\prime} \subset k^{\prime \prime}$, then the functor $\mathcal{R}\left(k^{\prime} ; R\right) \rightarrow \mathcal{R}\left(k^{\prime \prime} ; R\right)$ simply restricts the Galois action. With these functors, the family $\left(\mathcal{R}\left(k^{\prime} ; R\right)\right)$ forms a direct system. Every object of the direct limit category is represented by an object $X$ of $\mathcal{R}\left(k^{\prime} ; R\right)$ for some $k^{\prime}$. If objects $X, Y$ of $\mathcal{R}(k ; R)$ are represented by $X^{\prime}, Y^{\prime}$ in $\mathcal{R}\left(k^{\prime} ; R\right)$, then

$$
\operatorname{Hom}_{\mathcal{R}(k ; R)}(X, Y)=\bigcup_{k^{\prime} \subset k^{\prime \prime} \subset k} \operatorname{Hom}_{\mathcal{R}\left(k^{\prime \prime} ; R\right)}\left(X^{\prime \prime}, Y^{\prime \prime}\right)
$$

where $X^{\prime \prime}, Y^{\prime \prime}$ are the images of $X^{\prime}, Y^{\prime}$ in $\mathcal{R}\left(k^{\prime \prime} ; R\right)$.

Remark 1.3. Let $\Gamma$ be a profinite group. Two continuous linear representations $\rho_{1}$ and $\rho_{2}$ of open subgroups $U_{1}$ and $U_{2}$ of $\Gamma$ on an $R$-module $M$ are said to be related if they agree on an open subgroup of $U_{1} \cap U_{2}$. This is an equivalence relation, and an equivalence class will be called a germ of a continuous linear representation of $\Gamma$. A morphism $\left(M,\left[\rho_{U}\right]\right) \rightarrow\left(M^{\prime},\left[\rho_{U^{\prime}}^{\prime}\right]\right)$ of germs is an $R$-linear map $\alpha: M \rightarrow M^{\prime}$ that is equivariant for some open subgroup of $U \cap U^{\prime}$. When $k$ is a separable closure of a field $k_{0}$ finitely generated over the prime field, an object of $\mathcal{R}(k ; R)$ can be identified with a germ of a continuous linear representation of $\operatorname{Gal}\left(k / k_{0}\right)$.

Lemma 1.4. For each object $M$ of $\mathcal{R}\left(\Gamma ; \mathbb{Z}_{\ell}\right)$, there is an open subgroup $U \subset \Gamma$ such that $M$ decomposes into the sum of a torsion object and a torsion-free object in $\mathcal{R}\left(U ; \mathbb{Z}_{\ell}\right)$. Consequently, when $k$ is separably closed, for each $M$ in $\mathcal{R}\left(k ; \mathbb{Z}_{\ell}\right)$, $M_{\text {tors }}$ is a direct summand of $M$. 
Proof. Let $M=M_{\text {tors }} \oplus M_{1}$ as $\mathbb{Z}_{\ell}$-modules, and let $\gamma \in \Gamma$ act on $M$ as the matrix $\left(\begin{array}{cc}a(\gamma) & b(\gamma) \\ 0 & c(\gamma)\end{array}\right)$. The map $\gamma \mapsto a(\gamma)$ is the homomorphism describing the action of $\Gamma$ on $M_{\text {tors }}$. After replacing $\Gamma$ with an open subgroup $U$, we may suppose this action to be trivial. The map $\gamma \mapsto c(\gamma)$ defines an action of $U$ on $M_{1}$, and hence an action of $U$ on $\operatorname{Hom}\left(M_{1}, M_{\text {tors }}\right)$, which, again, we may suppose to be trivial. Then $\gamma \mapsto b(\gamma)$ is a homomorphism $U \rightarrow \operatorname{Hom}\left(M_{1}, M_{\text {tors }}\right)$, and after we replace $U$ with the kernel of this map, $M=M_{\text {tors }} \oplus M_{1}$ will be a decomposition of $U$-modules.

For $M$ in $\mathcal{R}\left(\Gamma ; \mathbb{Z}_{\ell}\right)$, set $M^{\vee}=\operatorname{Hom}_{\mathbb{Z}_{\ell}}\left(M, \mathbb{Z}_{\ell}\right)$ with $\gamma \in \Gamma$ acting according to the rule $(\gamma f)(m)=f\left(\gamma^{-1} m\right)$. We say that $M$ is reflexive if the canonical map $M \rightarrow M^{\vee \vee}$ is an isomorphism.

Proposition 1.5. (a) An object of $\mathcal{R}\left(\Gamma ; \mathbb{Z}_{\ell}\right)$ is reflexive if and only if it is torsionfree.

(b) Every object of $\mathcal{R}\left(\Gamma ; \mathbb{Z}_{\ell}\right)$ is a quotient of a reflexive object.

Proof. (a) As $M^{\vee}$ is torsion-free, the condition is necessary, and it is obviously sufficient.

(b) If $M$ decomposes in $\mathcal{R}\left(\Gamma ; \mathbb{Z}_{\ell}\right)$ into the direct sum of a torsion module $M_{t}$ with trivial $\Gamma$-action and a torsion-free module $M_{1}$, then $M$ will be the quotient of $\mathbb{Z}_{\ell}^{r} \oplus M_{1}$ for some $r$. According to the lemma, there is an open subgroup $U$ of $\Gamma$ such that $M$ has such a decomposition in $\mathcal{R}\left(U ; \mathbb{Z}_{\ell}\right)$, and hence there will be a surjection $\mathbb{Z}_{\ell}^{r} \oplus M_{1} \rightarrow M$ in $\mathcal{R}\left(U ; \mathbb{Z}_{\ell}\right)$ with $M_{1}$ free. Now

$$
\operatorname{Ind}_{U}^{\Gamma}\left(\mathbb{Z}_{\ell}^{r} \oplus M_{1}\right) \rightarrow \operatorname{Ind}_{U}^{\Gamma}(M) \stackrel{\varphi \mapsto \sum_{s \in \Gamma / U} s \varphi\left(s^{-1}\right)}{\longrightarrow} M
$$

realizes $M$ as the quotient of a torsion-free $\Gamma$-module.

Remark 1.6. (a) The category $\mathcal{R}\left(k ; \mathbb{Q}_{\ell}\right)$ is denoted Tate $(k)$ in Saavedra [47, VI, A4.2, A4.3], and its objects are called Tate modules. It is a neutral Tannakian category over $\mathbb{Q}_{\ell}$.

(b) The functor $M \mapsto\left(M_{\ell}\right)_{\ell \neq p}: \mathcal{R}\left(\Gamma ; \mathbb{Z}^{p}\right) \rightarrow \prod_{\ell \neq p} \mathcal{R}\left(\Gamma ; \mathbb{Z}_{l}\right)$ is exact, full, and faithful, with essential image the objects $\left(M_{\ell}\right)_{\ell \neq p}$ such that $\operatorname{dim}_{\mathbb{F}_{\ell}} M_{\ell} / \ell M_{\ell}$ is bounded.

The categories $\mathcal{R}$ at $p$. An $F$-isocrystal over a perfect field $k$ is a finite-dimensional $B(k)$-vector space $M$ endowed with a $\sigma$-linear bijection $F_{M}: M \rightarrow M$. With the obvious structures, the $F$-isocrystals over $k$ form a Tannakian category $\operatorname{Isoc}(k)$ over $\mathbb{Q}_{p}$, and the forgetful functor is a $B(k)$-valued fibre functor [47, VI, 3.2]. The identity object is $\mathbb{1}=(B(k), \sigma)$, and the Tate object is $\mathbb{T}=\left(B(k), p^{-1} \cdot \sigma\right)$.

An $F$-isocrystal $\left(M, F_{M}\right)$ is effective if $F_{M}$ stabilizes a $W(k)$-lattice in $M$. Let Isoc $^{+}(k)$ denote the full subcategory of $\operatorname{Isoc}(k)$ whose objects are the effective $F$-isocrystals. Every object of $\operatorname{Isoc}(k)$ is of the form $X \otimes \mathbb{T}^{\otimes m}$ with $X$ effective. In other words, for each $\left(M, F_{M}\right)$ in $\operatorname{Isoc}(k)$, there exists an $m \in \mathbb{Z}$ such that $p^{m} F_{M}$ stabilizes a lattice [47, VI, 3.1.3, 3.2.1].

An $F$-crystal 2 over a perfect field $k$ is a finitely generated $W(k)$-module $\Lambda$ endowed with a $\sigma$-linear map $F_{\Lambda}: \Lambda \rightarrow \Lambda$ such that the kernel of $F_{\Lambda}$ is torsion.

\footnotetext{
2Some authors use "crystal" to mean free crystal, i.e., an $M$ that is torsion-free (as a $W(k)$ module).
} 
The $F$-crystals over $k$ form an abelian tensor category $\operatorname{Crys}^{+}(k)$ over $\mathbb{Z}_{p}$. For an $F$-crystal $\left(\Lambda, F_{\Lambda}\right),\left(\Lambda, F_{\Lambda}\right)_{\mathbb{Q}}={ }_{\mathrm{df}}\left(\Lambda_{\mathbb{Q}}, F_{\Lambda} \otimes 1_{\mathbb{Q}}\right)$ is an $F$-isocrystal, and the functor

$$
\left(\Lambda, F_{\Lambda}\right) \mapsto\left(\Lambda, F_{\Lambda}\right)_{\mathbb{Q}}: \operatorname{Crys}^{+}(k)_{\mathbb{Q}} \rightarrow \operatorname{Isoc}(k)
$$

is fully faithful with essential image $\operatorname{Isoc}^{+}(k)$.

Let $\mathbb{L}=(W(k), p \cdot \sigma)$ be the Lefschetz $F$-crystal. The functor

$$
-\otimes \mathbb{L}: \operatorname{Crys}^{+}(k) \rightarrow \operatorname{Crys}^{+}(k)
$$

is faithful (full and faithful on torsion-free objects). We define Crys $(k)$ to be the tensor $\mathbb{Z}_{p}$-category obtained from $\operatorname{Crys}^{+}(k)$ by inverting $\mathbb{L}$. Thus, the objects of Crys $(k)$ are pairs $(\Lambda, m)$ with $\Lambda$ an $F$-crystal and $m \in \mathbb{Z}$, and

$$
\operatorname{Hom}\left((\Lambda, m),\left(\Lambda^{\prime}, m^{\prime}\right)\right)=\underset{N \geq m, m^{\prime}}{\lim _{N}} \operatorname{Hom}\left(\Lambda \otimes \mathbb{L}^{\otimes N-m}, \Lambda^{\prime} \otimes \mathbb{L}^{\otimes N-m^{\prime}}\right) .
$$

The tensor product is defined by

$$
(\Lambda, m) \otimes\left(\Lambda^{\prime}, m^{\prime}\right)=\left(\Lambda \otimes_{W(k)} \Lambda^{\prime}, m+m^{\prime}\right) .
$$

The Tate object $\mathbb{T}$ of $\operatorname{Crys}(k)$ is $(\mathbb{1}, 1)$.

The functor

$$
\Lambda \mapsto(\Lambda, 0): \operatorname{Crys}^{+}(k) \rightarrow \operatorname{Crys}(k)
$$

is faithful, and it is full on torsion-free objects. The forgetful functor defines an equivalence of the full subcategory of $\operatorname{Crys}(k)$ of torsion objects with the category of $W(k)$-modules of finite length.

Lemma 1.7. For each object $\Lambda$ of $\operatorname{Crys}^{+}(k)$, there is an $n$ such that $\Lambda \otimes \mathbb{L}^{\otimes n}$ decomposes into a direct sum of a torsion object and a torsion-free object. Consequently, for each $\Lambda$ in $\operatorname{Crys}(k), \Lambda_{\text {tors }}$ is a direct summand of $\Lambda$.

Proof. Let $\Lambda=\Lambda_{\text {tors }} \oplus \Lambda_{1}$ as $W(k)$-modules, and let $F_{\Lambda}$ act as $\left(\begin{array}{ll}a & b \\ 0 & c\end{array}\right)$. Then $F_{\Lambda \otimes \mathbb{L} \otimes n}$ acts as $\left(\begin{array}{cc}p^{n} a & p^{n} b \\ 0 & p^{n} c\end{array}\right)$. Choose $n$ so that $p^{n} \Lambda_{\text {tors }}=0$, and then $p^{n} b=0$. This proves the first statement, and the second follows because $-\otimes \mathbb{L}$ is an equivalence of categories on $\operatorname{Crys}(k)$.

Let $X=(\Lambda, m)$ and $X^{\prime}=\left(\Lambda^{\prime}, m^{\prime}\right)$ be objects of $\operatorname{Crys}(k)$. For some $N$ there exists a $\sigma^{-1}$-linear map $V_{\Lambda}: \Lambda \rightarrow \Lambda$ such that $F_{\Lambda} V_{\Lambda}=p^{N}=V_{\Lambda} F_{\Lambda}$. Let

$$
\underline{\operatorname{Hom}}\left(X, X^{\prime}\right)=\left(\operatorname{Hom}\left(\Lambda, \Lambda^{\prime}\right), N-m+m^{\prime}\right)
$$

with $\left(F_{\operatorname{Hom}\left(\Lambda, \Lambda^{\prime}\right)} f\right)(\lambda)=F_{\Lambda^{\prime}}\left(f\left(V_{\Lambda} \lambda\right)\right)$. This is an object of $\operatorname{Crys}(k)$ that is independent of the choice of $N$ (up to a canonical isomorphism). Define $X^{\vee}=\operatorname{Hom}(X, \mathbb{1})$ and say that $X$ is reflexive if the canonical map $X \rightarrow X^{\vee \vee}$ is an isomorphism.

Proposition 1.8. (a) An object of $\operatorname{Crys}(k)$ is reflexive if and only if it is torsionfree.

(b) Every object of $\operatorname{Crys}(k)$ is a quotient of a reflexive object.

(c) With the evaluation map $(f, x) \mapsto f(x): \underline{\operatorname{Hom}}\left(X, X^{\prime}\right) \otimes X \rightarrow X^{\prime}, \underline{\operatorname{Hom}}\left(X, X^{\prime}\right)$ is the internal Hom of $X$ and $X^{\prime}$ (in the sense of [47, I, 3.1.1]).

Proof. (a) As $X^{\vee}$ is torsion-free, the condition is necessary, and it is obviously sufficient.

(b) After (1.7) it suffices to prove this for a torsion $F$-crystal $\Lambda$. Such a $\Lambda$ is a quotient $M \rightarrow \Lambda$ of a free finitely generated $W$-module $M$. Endow $M$ with the 
$F$-crystal structure such that $F_{M}=\sigma$. After $M \rightarrow \Lambda$ has been tensored with a high power of $\mathbb{L}$, it will be a morphism of $F$-crystals.

(c) It is easy to check that this does have the correct universal property.

Remark 1.9. (a) The category Crys $^{+}(k)$ does not have internal Homs. For example, $\mathbb{L}^{\vee}=\underline{\operatorname{Hom}}(\mathbb{L}, \mathbb{1})$ does not exist in $\operatorname{Crys}^{+}(k)$.

(b) There is a canonical functor $\lim _{\longrightarrow} \operatorname{Isoc}\left(\mathbb{F}_{q}\right) \rightarrow \operatorname{Isoc}(\mathbb{F})$, which is faithful and essentially surjective [11, p. 85] but not full $\left(\right.$ note that $W(\mathbb{F}) \neq \lim W\left(\mathbb{F}_{q}\right)$ ). The category $\lim \operatorname{Isoc}\left(\mathbb{F}_{q}\right)$ is not semisimple, but, according to a theorem of Manin [11. p. 85], the category $\operatorname{Isoc}(\mathbb{F})$ is semisimple.

(c) Ekedahl [13, p. 36] defines a virtual $F$-crystal to be a triple $(M, F, \Lambda)$ with $(M, F)$ an isocrystal and $\Lambda$ a $W$-submodule of $M$ such that $B(k) \cdot \Lambda=M$; a virtual $F$-crystal is of finite type if $\Lambda$ is finitely generated. The functor $\left(\left(\Lambda, F_{\Lambda}\right), m\right) \mapsto$ $\left(\Lambda_{\mathbb{Q}}, F_{\Lambda} \otimes p^{-m}, \Lambda\right)$ defines an equivalence of the full subcategory of $\operatorname{Crys}(k)$ of torsion-free objects with the category of virtual $F$-crystals of finite type.

Definition 1.10. Let $k$ be a field of characteristic $p \neq 0$. If $k$ is finitely generated over $\mathbb{F}_{p}$, then

$$
\mathcal{R}\left(k ; \mathbb{Q}_{p}\right)=\operatorname{Isoc}\left(k^{\mathrm{pf}}\right), \quad \mathcal{R}\left(k ; \mathbb{Z}_{p}\right)=\operatorname{Crys}\left(k^{\mathrm{pf}}\right) .
$$

Otherwise,

$$
\mathcal{R}\left(k ; \mathbb{Z}_{p}\right)=\underset{k^{\prime} \subset k}{\lim } \mathcal{R}\left(k^{\prime} ; \mathbb{Z}_{p}\right), \quad \mathcal{R}\left(k ; \mathbb{Q}_{p}\right)=\underset{k^{\prime} \subset k}{\lim } \mathcal{R}\left(k^{\prime} ; \mathbb{Q}_{p}\right),
$$

where the limits are over the subfields of $k$ finitely generated over $\mathbb{F}_{p}$. We define $\mathcal{R}^{+}\left(k ; \mathbb{Q}_{p}\right)$ and $\mathcal{R}^{+}\left(k ; \mathbb{Z}_{p}\right)=\underline{\lim }_{k^{\prime} \subset k} \operatorname{Crys}^{+}\left(k^{\prime \text { pf }}\right)$ similarly.

Again, the direct limits of categories are meant in the naive sense (1.2).

\section{The categories $\mathcal{R}$ in general.}

Definition 1.11. For a field $k$ of characteristic $p \neq 0$,

$$
\begin{aligned}
\mathcal{R}(k ; \hat{\mathbb{Z}}) & =\mathcal{R}\left(k ; \mathbb{Z}^{p}\right) \times \mathcal{R}\left(k ; \mathbb{Z}_{p}\right), \quad \mathcal{R}\left(k ; \mathbb{A}_{f}\right)=\mathcal{R}\left(k ; \mathbb{A}_{f}^{p}\right) \times \mathcal{R}\left(k ; \mathbb{Q}_{p}\right), \\
\mathcal{R}^{+}(k ; \hat{\mathbb{Z}}) & =\mathcal{R}\left(k ; \mathbb{Z}^{p}\right) \times \mathcal{R}^{+}\left(k ; \mathbb{Z}_{p}\right), \quad \mathcal{R}^{+}\left(k ; \mathbb{A}_{f}\right)=\mathcal{R}\left(k ; \mathbb{A}_{f}^{p}\right) \times \mathcal{R}^{+}\left(k ; \mathbb{Q}_{p}\right) .
\end{aligned}
$$

For a field of characteristic zero,

$$
\mathcal{R}(k ; \hat{\mathbb{Z}})=\mathcal{R}\left(k ; \mathbb{Z}^{p}\right), \quad \mathcal{R}\left(k ; \mathbb{A}_{f}\right)=\mathcal{R}\left(k ; \mathbb{A}_{f}^{p}\right) .
$$

Note that $\mathcal{R}\left(k ; \mathbb{A}_{f}\right) \cong \mathcal{R}(k ; \hat{\mathbb{Z}})_{\mathbb{Q}}$. For notational convenience, we sometimes use $\mathcal{R}^{+}\left(k ; \mathbb{Z}_{l}\right)$ to denote $\mathcal{R}\left(k ; \mathbb{Z}_{l}\right)$ when $l \neq p$.

Each of the categories $\mathcal{R}(k ; R)$ has a natural tensor structure (see (3) for $\left.\mathcal{R}\left(k ; \mathbb{Z}_{p}\right)\right)$ for which it becomes a tensor category over $R$.

\footnotetext{
${ }^{3}$ For example, let $A_{1}$ and $A_{2}$ be ordinary elliptic curves over $\mathbb{F}_{q}$, and let $\Lambda_{1}$ and $\Lambda_{2}$ be their Dieudonné isocrystals. Then $\operatorname{Hom}\left(A_{1}, A_{2}\right) \otimes \mathbb{Q}_{p} \cong \operatorname{Hom}\left(\Lambda_{1}, \Lambda_{2}\right)$ (Tate's theorem). When we pass to the limit over increasing $q$ we get an isomorphism $\operatorname{Hom}\left(A_{1 / \mathbb{F}}, A_{2 / \mathbb{F}}\right) \otimes \mathbb{Q}_{p} \cong$ $\operatorname{Hom}_{\lim } \operatorname{Isoc}\left(\mathbb{F}_{q}\right)\left(\Lambda_{1}, \Lambda_{2}\right)$. On the other hand, $\operatorname{Hom}_{\operatorname{Isoc}(\mathbb{F})}\left(\Lambda_{1}, \Lambda_{2}\right) \approx \mathbb{Q}_{p} \oplus \mathbb{Q}_{p}$. Thus, if $A_{1}$ and $A_{2}$ have different $j$-invariants, $\operatorname{Hom}_{\underline{\lim }} \operatorname{Isoc}\left(\mathbb{F}_{q}\right)\left(\Lambda_{1}, \Lambda_{2}\right)=0 \neq \operatorname{Hom}_{\operatorname{Isoc}(\mathbb{F})}\left(\Lambda_{1}, \Lambda_{2}\right)$.
} 
The motivic subcategory of $\mathcal{R}\left(k ; \mathbb{Q}_{\ell}\right)$. Let $\mathcal{M}\left(k ; \mathbb{Q}_{\ell}\right)$ be the strictly full subcategory of $\mathcal{R}\left(k ; \mathbb{Q}_{\ell}\right)$ whose objects are subquotients of a Tate twist of a tensor power of an $\ell$-adic étale cohomology group of a smooth projective variety over $k$. When $k=\mathbb{F}_{q}, \mathcal{M}\left(k ; \mathbb{Q}_{\ell}\right)$ includes the category of semisimple representations of $\Gamma=\operatorname{Gal}\left(k^{\mathrm{al}} / k\right)$ for which the eigenvalues of the Frobenius generator of $\Gamma$ are Weil $q$-numbers, and conjecturally equals it: all such representations arise already in the cohomology of abelian varieties (cf. [40,3.7]), and the Weil conjectures (proved by Grothendieck and Deligne) show that the Frobenius eigenvalues of objects in $\mathcal{M}\left(k ; \mathbb{Q}_{\ell}\right)$ are Weil $q$-numbers; the semisimplicity is a conjecture. When $k$ is a number field, Conjecture 1 of [15] suggests a description of $\mathcal{M}\left(k ; \mathbb{Q}_{\ell}\right)$.

\section{Adèlic COHOMOLOGY}

Modules over $\mathbb{A}_{f}^{p}$ and lattices. Recall (Notations, p. 500) that $\mathbb{Z}^{p}=\prod_{\ell \neq p} \mathbb{Z}_{\ell}$ and $\mathbb{A}_{f}^{p}$ is the restricted direct product $\mathbb{A}_{f}^{p}=\prod_{\ell \neq p}\left(\mathbb{Q}_{\ell}, \mathbb{Z}_{\ell}\right)$.

2.1. Endow $\left(\mathbb{A}_{f}^{p}\right)^{m}$ with its topology as the restricted direct product of the spaces $\mathbb{Q}_{\ell}^{m}$ relative to the subspaces $\mathbb{Z}_{\ell}^{m}$. Every $\mathbb{A}_{f}^{p}$-linear bijection $\left(\mathbb{A}_{f}^{p}\right)^{m} \rightarrow\left(\mathbb{A}_{f}^{p}\right)^{m}$ is bicontinuous. This allows us to endow any free $\mathbb{A}_{f}^{p}$-module $M$ of finite rank with a topology by choosing an isomorphism $\alpha: M \rightarrow\left(\mathbb{A}_{f}^{p}\right)^{m}$.

2.2. Let $M$ be a free $\mathbb{A}_{f}^{p}$-module of finite rank $m$, so that $M_{\ell}={ }_{\mathrm{df}} M \otimes_{\mathbb{A}_{f}^{p}} \mathbb{Q}_{\ell}$ is a $\mathbb{Q}_{\ell}$-vector space of dimension $m$ for all $\ell \neq p$. A lattice in $M$ is a finitely generated $\mathbb{Z}^{p}$-submodule $\Lambda$ such that the inclusion $\Lambda \hookrightarrow M$ induces an isomorphism $\Lambda_{\mathbb{A}_{f}^{p}} \rightarrow M$; then $\Lambda_{\ell}={ }_{\mathrm{df}} \Lambda \otimes_{\mathbb{Z}^{p}} \mathbb{Z}_{\ell}$ is a $\mathbb{Z}_{\ell^{-}}$lattice in $M_{\ell}$ for all $\ell \neq p$, and $\Lambda$ is the free $\mathbb{Z}^{p}$-submodule spanned by a basis for $M$. Clearly, every lattice in $M$ is compact and open, and so any two lattices $\Lambda$ and $\Lambda^{\prime}$ in $M$ are commensurable, that is, both quotients $\Lambda / \Lambda \cap \Lambda^{\prime}$ and $\Lambda^{\prime} / \Lambda \cap \Lambda^{\prime}$ are finite (hence, $\Lambda_{\ell}=\Lambda_{\ell}^{\prime}$ for almost all $\ell$ ). Let $M_{0}$ be a $\mathbb{Q}$-structure on $M$ (i.e., a $\mathbb{Q}$-subspace such that the inclusion $M_{0} \hookrightarrow M$ induces an isomorphism $\left.M \otimes_{\mathbb{Q}} \mathbb{A}_{f}^{p} \rightarrow M\right)$. There is a canonical one-to-one correspondence between the lattices $\Lambda$ in $M$ and the lattices $\Lambda_{0}$ in $M_{0}$, namely,

$$
\Lambda \mapsto \Lambda \cap M_{0}, \Lambda_{0} \mapsto \text { its closure in } M .
$$

2.3. Let $\left(M_{\ell}\right)_{\ell \neq p}$ be a family of vector spaces over the fields $\mathbb{Q}_{\ell}$, all of the same finite dimension. Two families $\left(\Lambda_{\ell}\right)_{\ell \neq p}$ and $\left(\Lambda_{\ell}^{\prime}\right)_{\ell \neq p}$ of lattices in the $M_{\ell}$ will be said to be equivalent if $\Lambda_{\ell}=\Lambda_{\ell}^{\prime}$ for almost all $\ell$. To give a free $\mathbb{A}_{f}^{p}$-module of finite rank is the same as to give a family $\left(M_{\ell}\right)_{\ell \neq p}$ together with an equivalence class of families $\left(\Lambda_{\ell}\right)_{\ell \neq p}$ : given a family $\left(M_{\ell}, \Lambda_{\ell}\right)_{\ell \neq p}$, let $M=\prod\left(M_{\ell}, \Lambda_{\ell}\right)$; given $M$, choose an isomorphism $M \rightarrow\left(\mathbb{A}_{f}^{p}\right)^{m}$, and let $\Lambda_{\ell}$ be the inverse image of $\mathbb{Z}_{\ell}^{m}$. Let $M$ and $M^{\prime}$ be the free $\mathbb{A}_{f}^{p}$-modules of finite rank defined by systems $\left(M_{\ell}, \Lambda_{\ell}\right)_{\ell \neq p}$ and $\left(M_{\ell}^{\prime}, \Lambda_{\ell}^{\prime}\right)_{\ell \neq p}$, respectively. A family of $\mathbb{Q}_{\ell}$-linear maps $\alpha_{\ell}: M_{\ell} \rightarrow M_{\ell}^{\prime}$ defines an $\mathbb{A}_{f}^{p}$-linear map $\alpha: M \rightarrow M^{\prime}$ if and only if $\alpha_{\ell}\left(\Lambda_{\ell}\right) \subset \Lambda_{\ell}^{\prime}$ for almost all $\ell$, in which case $\alpha$ is injective if and only if $\alpha_{\ell}$ is injective for all $\ell$ and it is surjective if and only if $\alpha_{\ell}$ is surjective for all $\ell$ and maps $\Lambda_{\ell}$ onto $\Lambda_{\ell}^{\prime}$ for almost all $\ell$.

2.4. An $\mathbb{A}_{f}^{p}$-linear map $\alpha: M \rightarrow M^{\prime}$ of free $\mathbb{A}_{f}^{p}$-modules of finite rank will be said to be of constant rank if the rank of $\alpha_{\ell}={ }_{\mathrm{df}} \alpha \otimes_{\mathbb{A}_{f}^{p}} \mathbb{Q}_{\ell}$ is independent of $\ell$. Then the kernel, cokernel, image, and co-image of $\alpha$ are again free $\mathbb{A}_{f}^{p}$-modules of finite rank. 
Adèlic cohomology (characteristic $p \neq 0$ ). Throughout this subsection, all varieties are smooth and projective over a separably closed field $k$ of characteristic $p \neq 0$. For such a variety $V, H^{r}(V,-)$ denotes an étale cohomology group and $\Lambda_{\ell}^{i}(V, r)$ denotes the image of $H^{i}\left(V, \mathbb{Z}_{\ell}(r)\right)$ in $H^{i}\left(V, \mathbb{Q}_{\ell}(r)\right)$. Thus,

$$
\Lambda_{\ell}^{i}(V, r) \cong H^{i}\left(V, \mathbb{Z}_{\ell}(r)\right) /\{\text { torsion }\} .
$$

Proposition 2.5. (a) The torsion subgroup of $H^{i}\left(V, \mathbb{Z}_{\ell}\right)$ is finite for all $\ell$, and is zero for almost all $\ell$.

(b) Assume $V$ is connected of dimension $d_{V}$, and fix a prime $\ell \neq p$. If the cohomology groups $H^{j}\left(V, \mathbb{Z}_{\ell}\right)$ of $V$ are torsion-free for all $j$, then the pairing

$$
H^{i}\left(V, \mathbb{Q}_{\ell}\right) \times H^{2 d_{V}-i}\left(V, \mathbb{Q}_{\ell}\left(d_{V}\right)\right) \rightarrow H^{2 d_{V}}\left(V, \mathbb{Q}_{\ell}\left(d_{V}\right)\right) \cong \mathbb{Q}_{\ell}
$$

induces a perfect $\mathbb{4}^{4}$ pairing on the cohomology groups with coefficients in $\mathbb{Z}_{\ell}$.

(c) Let $W$ be a second variety over $k$, and fix a prime $\ell \neq p$. If the groups $H^{i}\left(V, \mathbb{Z}_{\ell}\right)$ and $H^{j}\left(W, \mathbb{Z}_{\ell}\right)$ are torsion-free for all $i$ and $j$, then the Künneth isomorphism

$$
H^{r}\left(V \times W, \mathbb{Q}_{\ell}\right) \cong \bigoplus_{i+j=r} H^{i}\left(V, \mathbb{Q}_{\ell}\right) \otimes H^{j}\left(W, \mathbb{Q}_{\ell}\right)
$$

induces an isomorphism on the groups with coefficients in $\mathbb{Z}_{\ell}$.

Proof. (a) The finiteness of the torsion subgroup of $H^{i}\left(V, \mathbb{Z}_{\ell}\right)$ is a standard result. That the groups $H^{r}\left(V, \mathbb{Z}_{\ell}\right)$ are torsion-free for almost all $\ell$ is a theorem of Gabber 17.

(b) The pairing on the cohomology groups with coefficients in $\mathbb{Z}_{\ell}$ is the unique pairing for which the diagrams

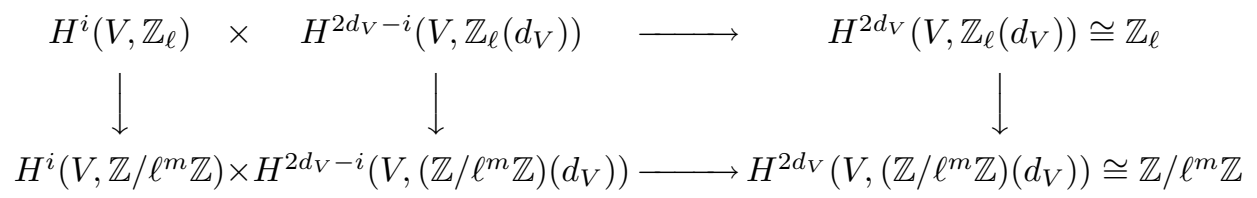

commute for all $m$. The lower pairing is perfect, and the vertical maps are the cokernels of multiplication by $\ell^{m}$ on $H^{i}\left(V, \mathbb{Z}_{\ell}\right)$ and $H^{2 d_{V}-i}\left(V, \mathbb{Z}_{\ell}\left(d_{V}\right)\right)$ because we are assuming there is no torsion. This implies (even with $m=1$ ) that the discriminant of the top pairing is an $\ell$-adic unit, and so the top pairing is also perfect 5

(c) This is a standard result.

Definition 2.6. For $V$ as above, $H^{i}\left(V, \mathbb{A}_{f}^{p}(r)\right)$ is the restricted direct product of the spaces $H^{i}\left(V, \mathbb{Q}_{\ell}(r)\right)$ with respect to the lattices $\Lambda^{i}(V, r)$. Equivalently,

$$
H^{i}\left(V, \mathbb{A}_{f}^{p}(r)\right)=H^{i}\left(V, \mathbb{Z}^{p}(r)\right) \otimes \mathbb{Q}
$$

where $H^{i}\left(V, \mathbb{Z}^{p}(r)\right)=\varliminf_{(n, p)=1} H^{i}\left(V, \mu_{n}^{\otimes r}\right)$.

Remark 2.7. (a) Because the Betti numbers of a smooth projective variety are independent of $\ell, H^{i}\left(V, \mathbb{A}_{f}^{p}\right)$ is a free $\mathbb{A}_{f}^{p}$-module of finite rank. A regular map $\alpha: V \rightarrow W$ defines an $\mathbb{A}_{f}^{p}$-linear map $\alpha^{*}: H^{i}\left(W, \mathbb{A}_{f}^{p}\right) \rightarrow H^{i}\left(V, \mathbb{A}_{f}^{p}\right)$.

(b) For a connected $V$, the proposition implies the following:

\footnotetext{
${ }^{4}$ A bilinear form $M \times N \rightarrow R$ of $R$-modules is nondegenerate if its left and right kernels are zero, and perfect if the maps $M \rightarrow \operatorname{Hom}_{R}(N, R)$ and $N \rightarrow \operatorname{Hom}_{R}(M, R)$ it defines are isomorphisms.

${ }^{5}$ We thank M. Nori for this proof, which is simpler than our original.
} 
the pairings in (b) of the proposition define a perfect pairing

$$
H^{i}\left(V, \mathbb{A}_{f}^{p}\right) \times H^{2 d_{V}-i}\left(V, \mathbb{A}_{f}^{p}\left(d_{V}\right)\right) \rightarrow H^{2 d_{V}}\left(V, \mathbb{A}_{f}^{p}\left(d_{V}\right)\right) \cong \mathbb{A}_{f}^{p}
$$

of free $\mathbb{A}_{f}^{p}$-modules of finite rank.

In other words, Poincaré duality holds for $\mathbb{A}_{f}^{p}$-cohomology. This implies that the map $\alpha^{*}$ in (a) has an $\mathbb{A}_{f}^{p}$-linear adjoint

$$
\alpha_{*}: H^{j}\left(V, \mathbb{A}_{f}^{p}\left(d_{V}\right)\right) \rightarrow H^{j+2 d_{W}-2 d_{V}}\left(W, \mathbb{A}_{f}^{p}\left(d_{W}\right)\right), \quad j=2 d_{V}-i .
$$

(c) Part (c) of the theorem implies the following: there is a canonical isomorphism of free $\mathbb{A}_{f}^{p}$-modules of finite rank

$$
H^{r}\left(V \times W, \mathbb{A}_{f}^{p}\right) \cong \bigoplus_{i+j=r} H^{i}\left(V, \mathbb{A}_{f}^{p}\right) \otimes H^{j}\left(W, \mathbb{A}_{f}^{p}\right)
$$

(d) Assume (for simplicity) that $V$ is connected. Let $f$ be an algebraic correspondence of degree $r$ from $V$ to $W$ with rational coefficients, i.e., an element of $Z^{d_{V}+r}(V \times W)_{\mathbb{Q}}$. For all but finitely many $\ell, f$ will be $\ell$-integral, and so its $\ell$-adic cohomology classes define an element $c l(f) \in H^{2 d_{V}+2 r}\left(V \times W, \mathbb{A}_{f}^{p}\left(d_{V}+r\right)\right)$. Combining this remark with (b) and (c), we obtain a map (also denoted $f$ )

$$
x \mapsto q_{W *}\left(c l(f) \cup q_{V}^{*} x\right): H^{i}\left(V, \mathbb{A}_{f}^{p}(m)\right) \rightarrow H^{i+2 r}\left(W, \mathbb{A}_{f}^{p}(m+r)\right)
$$

for all $m$. Here $q_{W}$ and $q_{V}$ are the projection morphisms.

Adèlic cohomology (characteristic zero). Let $V$ be a variety (not necessarily smooth or projective) over an algebraically closed field $k$ of characteristic zero. For any model $V_{0} / k_{0}$ of $V$ over an algebraically closed subfield $k_{0}$ of $k$ and embedding $k_{0} \hookrightarrow \mathbb{C}$,

$$
H^{i}\left(V, \mathbb{Z}_{l}\right) \cong H^{i}\left(V_{0}, \mathbb{Z}_{l}\right) \cong H^{i}\left(V_{0}(\mathbb{C}), \mathbb{Z}\right)_{\mathbb{Z}_{l}}
$$

The group $H^{i}\left(V_{0}(\mathbb{C}), \mathbb{Z}\right)$ is finitely generated, and so $H^{i}\left(V, \mathbb{Z}_{l}\right)$ is torsion-free for almost all $l$. The results in the last subsection hold mutatis mutandis for the group $H^{i}\left(V, \mathbb{A}_{f}(r)\right)$ defined to be the restricted topological product of the $H^{i}\left(V, \mathbb{Q}_{l}\right)$ with respect to the $\Lambda_{l}^{i}(V, r)$. For any choice of a pair $V_{0} / k_{0}, k_{0} \hookrightarrow \mathbb{C}$ as above,

$$
H^{i}\left(V, \mathbb{A}_{f}\right) \cong H^{i}\left(V_{0}, \mathbb{A}_{f}\right) \cong H^{i}\left(V_{0}(\mathbb{C}), \mathbb{Q}\right)_{\mathbb{A}_{f}} .
$$

\section{An ADÈlic TATe COnjecture}

Throughout this section, $V$ is a smooth projective variety of dimension $d$ over a field $k$. For $\ell \neq \operatorname{char}(k), H^{i}\left(V, \mathbb{Z}_{\ell}(r)\right)$ denotes the étale cohomology group. We let $\bar{k}=k^{\text {sep }}$ and $\bar{V}=V_{/ \bar{k}}$. Finally, $\Gamma=\operatorname{Gal}(\bar{k} / k)$.

Conjectures on algebraic cycles in $\ell$-adic cohomology. The group $Z_{\ell \text {-hom }}^{r}(V)$ is the co-image of the cycle class map $Z^{r}(V) \rightarrow H^{2 r}\left(\bar{V}, \mathbb{Z}_{\ell}(r)\right)$, and $Z_{\text {num }}^{r}(V)$ is the quotient of $Z^{r}(V)$ by numerical equivalence.

Recall [55] that, when $k$ is finitely generated over the prime field, there are the following conjectures.

$\mathbf{T}^{r}(\mathbf{V} / \mathbf{k}, \ell):$ The cycle map $Z_{\ell \text {-hom }}^{r}(V)_{\mathbb{Q}_{\ell}} \rightarrow H^{2 r}\left(\bar{V}, \mathbb{Q}_{\ell}(r)\right)^{\Gamma}$ is surjective.

$\mathbf{E}^{r}(\mathbf{V} / \mathbf{k}, \ell)$ : The quotient map $Z_{\ell \text {-hom }}^{r}(V)_{\mathbb{Q}} \rightarrow Z_{\text {num }}^{r}(V)_{\mathbb{Q}}$ is an isomorphism.

$\mathbf{I}^{r}(\mathbf{V} / \mathbf{k}, \ell)$ : The cycle map $Z_{\ell \text {-hom }}^{r}(V)_{\mathbb{Q}_{\ell}} \rightarrow H^{2 r}\left(\bar{V}, \mathbb{Q}_{\ell}(r)\right)$ is injective.

$\mathbf{S}^{r}(\mathbf{V} / \mathbf{k}, \ell)$ : The map $H^{2 r}\left(\bar{V}, \mathbb{Q}_{\ell}(r)\right)^{\Gamma} \rightarrow H^{2 r}\left(\bar{V}, \mathbb{Q}_{\ell}(r)\right)_{\Gamma}$ induced by the identity map is bijective. 


\section{An adèlic Tate conjecture.}

Lemma 3.1. Assume $k$ is finitely generated over the prime field. If $T^{r}(V / k, \ell)$, $T^{d-r}(V / k, \ell)$, and $S^{r}(V / k, \ell)$ hold for almost all $\ell$, then the cycle class map

$$
Z_{\ell-\mathrm{hom}}^{r}(V)_{\mathbb{Z}_{\ell}} \rightarrow H^{2 r}\left(\bar{V}, \mathbb{Z}_{\ell}(r)\right)^{\Gamma}
$$

is surjective for almost all $\ell$.

Proof. According to [55, 2.9], the conditions imply that $E^{r}(V / k, \ell)$ and $E^{d-r}(V / k, \ell)$ hold for almost all $\ell$.

Note that $E^{r}(V / k, \ell)$ implies $Z_{\ell \text {-hom }}^{r}(V) /\{$ torsion $\} \cong Z_{\text {num }}^{r}(V)$, which is finitely generated (see, for example, [37, VI, 11.7]). Therefore, for those $\ell$ for which $E^{r}(V / k, \ell)$ and $E^{d-r}(V / k, \ell)$ hold,

$$
Z_{\ell \text {-hom }}^{r}(V) /\{\text { torsion }\} \times Z_{\ell \text {-hom }}^{d-r}(V) /\{\text { torsion }\} \rightarrow \mathbb{Z}
$$

is a nondegenerate pairing of finitely generated abelian groups whose discriminant $D$ is independent of $\ell$.

Consider the diagram

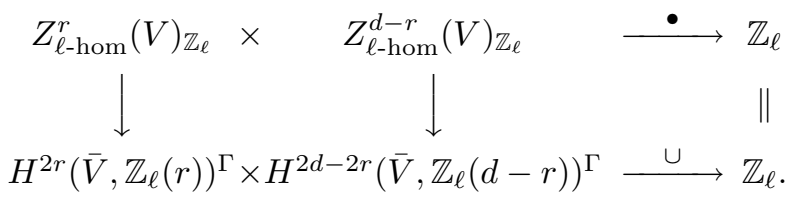

For those $\ell$ for which $T^{r}(V / k, \ell)$ and $T^{d-r}(V / k, \ell)$ hold, the vertical arrows have finite cokernel.

For those $\ell$ for which

- the groups $H^{2 r}\left(\bar{V}, \mathbb{Z}_{\ell}(r)\right)$ and $H^{2 d-2 r}\left(\bar{V}, \mathbb{Z}_{\ell}(d-r)\right)$ are torsion-free,

- $E^{r}(V / k, \ell)$ and $E^{d-r}(V / k, \ell)$ hold,

- $T^{r}(V / k, \ell)$ and $T^{d-r}(V / k, \ell)$ hold, and

- $\ell$ does not divide $D$,

the vertical maps are surjective. Since these conditions hold for almost all primes $\ell$, this proves the lemma.

Theorem 3.2. Let $k$ be as in the lemma. If $T^{r}(V / k, \ell), T^{d-r}(V / k, \ell)$, and $S^{r}(V / k, \ell)$ hold for all $\ell \neq p$, then the cycle class map defines a bijection

$$
Z_{\text {num }}^{r}(V)_{\mathbb{A}_{f}^{p}} \rightarrow H^{2 r}\left(\bar{V}, \mathbb{A}_{f}^{p}(r)\right)^{\Gamma} .
$$

Proof. We have to show that the cycle class map $Z^{r}(V) \rightarrow H^{2 r}\left(\bar{V}, \mathbb{Z}_{\ell}(r)\right)$ defines

(a) a bijection $Z_{\text {num }}^{r}(V)_{\mathbb{Q}_{\ell}} \rightarrow H^{2 r}\left(\bar{V}, \mathbb{Q}_{\ell}(r)\right)^{\Gamma}$ for all $\ell \neq p$, and

(b) a surjection $Z_{\text {num }}^{r}(V)_{\mathbb{Z}_{\ell}} \rightarrow \Lambda_{\ell}^{2 r}(\bar{V}, r)^{\Gamma}$ for almost all $\ell$.

Applying [55, 2.9], we see that $E^{r}(V / k, \ell), I^{r}(V / k, \ell)$, and $T^{r}(V / k, \ell)$ hold for all $\ell \neq p$. This implies (a), and (b) follows from the lemma.

Corollary 3.3. Let $V$ be a (smooth projective) variety over $\mathbb{F}_{q}$, and let $r$ be an integer. The following statements are equivalent:

(a) for some prime $\ell \neq p$, $\operatorname{dim}_{\mathbb{Q}} Z_{\text {num }}^{r}(V)=\operatorname{dim}_{\mathbb{Q}_{\ell}} H^{2 r}\left(V, \mathbb{Q}_{\ell}(r)\right)^{\Gamma}$;

(b) the order of the pole of the zeta function $Z(V, t)$ of $V$ at $t=q^{-r}$ is equal to the rank of $Z_{\text {num }}^{r}(V)$;

(c) the cycle class map defines a bijection $Z_{\text {num }}^{r}(V)_{\mathbb{A}_{f}^{p}} \rightarrow H^{2 r}\left(\bar{V}, \mathbb{A}_{f}^{p}(r)\right)^{\Gamma}$.

Proof. Apply [55, 2.9] and the theorem. 
Counterexamples to an integral Tate conjecture. Assume $k$ is finitely generated over the prime field, and consider the cycle class map

$$
Z^{r}(V)_{\mathbb{Z}_{\ell}} \rightarrow H^{2 r}\left(\bar{V}, \mathbb{Z}_{\ell}(r)\right)^{\Gamma} .
$$

If $k$ is infinite, there exist $V$ 's without a rational zero cycle of degree 1 (for example, any curve of genus zero without a rational point or any curve of genus 1 with index $\neq 1$ ) and for such a variety, (4) will fail to be surjective for $r=d$ and suitable $\ell$. The argument that Atiyah and Hirzebruch [3] used to prove that not all torsion cohomology classes on a complex variety are algebraic (contradicting Hodge's original conjecture) also applies over finite fields 7 and proves that (4) is not surjective on torsion classes.

Griffiths and Harris 20] conjecture that if $V$ is a very general hypersurface 8 of degree $d \geq 6$ in $\mathbb{P}^{4}$, then $d$ divides the degree of every curve on $V$. Kollár has obtained some results in the direction of this conjecture (see [4, p. 135]). Thus, it is known that, if $k$ is an uncountable algebraically closed field and $\ell>3$ is distinct from the characteristic of $k$, then there exist smooth hypersurfaces $V \subset \mathbb{P}^{4}$ such that the cycle class map

$$
Z^{2}(V)_{\mathbb{Z}_{\ell}} \rightarrow H^{4}\left(V, \mathbb{Z}_{\ell}(2)\right) \cong \mathbb{Z}_{\ell}
$$

is not surjective (cf. [48, 0.6]).

We do not know of an example with $k$ finite for which (44) is not surjective modulo torsion (however, Schoen [48, 2.1] gives an example with $V$ singular). For $r=1$ and $k$ finite, one sees from the Kummer sequence that $T^{1}(V / k, \ell)$ implies that the map (4) is surjective.

\section{The ADÈLIC FIBRE FUnCTOR}

Isomotives. Fix a field $k$ of characteristic exponent $p$, and let $\mathcal{S}$ be a class of smooth projective varieties over $k$ satisfying the following condition:

4.1. $\mathcal{S}$ is closed under passage to a connected component and under the formation of products and disjoint unions, and it contains the zero-dimensional varieties and the projective spaces.

4.2. Fix an adequate equivalence relation $\sim$. Recall that, for smooth projective varieties $V$ and $W$ over $k$, the group of correspondences of degree $i$ from $V$ to $W$ is

$$
\operatorname{Corr}^{i}(V, W)=\bigoplus_{j} Z_{\sim}^{\operatorname{dim}\left(V_{j}\right)+i}\left(V_{j} \times W\right)_{\mathbb{Q}}
$$

where the $V_{j}$ are the equidimensional components of $V$.

4.3. The category $\mathcal{C V}_{\sim}^{0}(k)$ of correspondences based on $\mathcal{S}$ has one object $h V$ for each $V \in \mathcal{S}$, and

With the definition

$$
\operatorname{Hom}_{\mathcal{C} \mathcal{V}_{\sim}^{0}(k)}(h V, h W)=\operatorname{Corr}^{0}(V, W)_{\mathbb{Q}} .
$$

$$
h V \otimes h W=h(V \times W)
$$

\footnotetext{
${ }^{6}$ For such a curve, even $C H^{1}(\bar{V})^{\Gamma} \stackrel{\text { degree }}{\longrightarrow} \mathbb{Z}$ fails to be surjective [33.

${ }^{7}$ For this, one needs to use that the odd-dimensional Steenrod operations with values in $H^{*}(\bar{V}, \mathbb{Z} / \ell \mathbb{Z})$ vanish on algebraic cycles $[6]$.

${ }^{8} \mathrm{~A}$ statement is said to hold for a very general hypersurface of degree $d$ if it holds for all hypersurfaces in the complement of countably many proper subvarieties of the space of hypersurfaces of degree $d$.
} 
and the obvious constraints, $\mathcal{C V}_{\sim}^{0}(k)$ becomes a tensor category over $\mathbb{Q}$ [47, VI, 4.1.1].

4.4. The category of effective isomotives $\mathcal{M}_{\sim}^{+}(k ; \mathbb{Q} ; \mathcal{S})$ is the pseudo-abelian hull of $\mathcal{C} \mathcal{V}_{\sim}^{0}(k)$. Its objects are pairs $(h V, e)$ with $e$ an idempotent in $\operatorname{End}(h V)$. It is a pseudo-abelian tensor category over $\mathbb{Q}$.

4.5. In $\mathcal{M}_{\sim}^{+}(k ; \mathbb{Q} ; \mathcal{S})$ there is a canonical decomposition

$$
h \mathbb{P}^{1}=\mathbb{1} \oplus \mathbb{L},
$$

and the category of isomotives $\mathcal{M}_{\sim}(k ; \mathbb{Q} ; \mathcal{S})$ is obtained from $\mathcal{M}_{\sim}^{+}(k ; \mathbb{Q} ; \mathcal{S})$ by inverting $\mathbb{L}$ [47. VI, 4.1.3.1]. Its objects are pairs $((h V, e), m)$ with $(h V, e)$ an effective motive and $m \in \mathbb{Z}$. We sometimes write $h(V, e, m)$ for $((h V, e), m)$. Note that $(V, e, m) \mapsto h(V, e, m)$ gives a one-to-one correspondence between the triples $\left(V \in \mathcal{S}, e \in \operatorname{End}(h V)\right.$ with $\left.e^{2}=e, m \in \mathbb{Z}\right)$ and the objects of $\mathcal{M}_{\sim}(k ; \mathbb{Q} ; \mathcal{S})$.

4.6. Let $V$ be a smooth projective variety of dimension $n$, and let $e_{l}^{i} \in H^{2 n-i}(V) \otimes$ $H^{i}(V)(n)$ be the $i^{\text {th }}$ Künneth component of the diagonal for the $l$-adic étale cohomology $(l \neq p)$ or the crystalline cohomology $(l=p \neq 1)$. We say that the Künneth projectors at $l$ are algebraic for $V$ if, for each $i$, there exists a $\pi_{l}^{i} \in \mathrm{Z}^{n}(V \times V)$ mapping to $e_{l}^{i}$. We note the following.

(a) If the ground field $k$ is finite, then $\pi_{l}^{i}$ always exists and is given by a polynomial $P^{i}$ with rational coefficients in the Frobenius endomorphism which is independent of $l$. This follows from knowing the Weil conjectures for the cohomologies in question [30, Theorem 2].

(b) If the Künneth projectors are algebraic, say, $\pi_{l}^{i} \mapsto e_{l}^{i}$, for several $l$, then the numerical equivalence classes of the $\pi_{l}^{i}$ are independent of $l$. In characteristic zero, this follows from a comparison with Betti cohomology. In nonzero characteristic, $V, \pi_{l}^{i}, \ldots$ will have a smooth specialization to a system $V_{0}, \pi_{l 0}^{i}, \ldots$ over a finite field. Recall that smooth specialization gives isomorphisms on the relevant cohomology groups. According to (a), $\pi_{l 0}^{i}$ differs from $P^{i}$ by an algebraic class that is $l$-homologically (hence, numerically) equivalent to zero. Therefore, the numerical equivalence class of $\pi_{l 0}^{i}$ is independent of $l$, and it follows that the same is true of the $\pi_{l}^{i}$ (cf. [2] Proposition 5]).

The category $\mathcal{M}_{\sim}(k ; \mathbb{Q} ; \mathcal{S})$ has a naive tensor structure for which it is a pseudoabelian rigid tensor category over $\mathbb{Q}$ [47, VI, 4.1.3.5], but it cannot be Tannakian. When the Künneth projectors are algebraic for all $V$ in $\mathcal{S}$ and (some or) all $l$, we have well-defined elements $\pi^{i} \in Z_{\mathrm{num}}^{\operatorname{dim} V}(V \times V)$, which we use to modify the commutativity constraints in $\mathcal{M}_{\text {num }}^{+}(k ; \mathbb{Q} ; \mathcal{S})$ and $\mathcal{M}_{\text {num }}(k ; \mathbb{Q} ; \mathcal{S}$ ) (see [47, VI, 4.2.1.4] for an explanation of this). With this new tensor structure, $\mathcal{M}_{\text {num }}(k ; \mathbb{Q} ; \mathcal{S})$ is Tannakian if and only if it is abelian.

4.7. If numerical equivalence on $Z^{*}(V)_{\mathbb{Q}}$ coincides with $l$-adic homological equivalence for all $V \in \mathcal{S}$, then the Künneth projectors at $l$ are algebraic for all $V \in \mathcal{S}$. More precisely, the hypothesis for $V \times V$ implies the conclusion for $V$ (31, 5-1, $4-1(1), 4-1(4)])$.

For the remainder of this section, we assume that $\sim$ is not coarser than $\ell$-adic homological equivalence on $Z^{r}(V)_{\mathbb{Q}}$ for any $\ell \neq p$, so that the formula

$$
\omega_{\ell}(h(V, e, m))=e\left(H^{*}\left(\bar{V}, \mathbb{Q}_{\ell}(m)\right)\right), \quad \bar{V}=V_{k^{\mathrm{sep}}},
$$


defines a functor $\omega_{\ell}: \mathcal{M}_{\sim}(k ; \mathbb{Q} ; \mathcal{S}) \rightarrow \operatorname{Vec}\left(\mathbb{Q}_{\ell}\right)$, and we drop $\sim$ from the notation $\mathbb{G}$

The adèlic fibre functor. With the notation of (2.6), define

$$
\omega^{p}(h V)=H^{*}\left(\bar{V}, \mathbb{A}_{f}^{p}\right)
$$

Because of (2.7d) and our assumption on $\sim$, a correspondence $f$ of degree 0 from $V$ to $W$ defines a homomorphism $\omega^{p}(f): \omega^{p}(h V) \rightarrow \omega^{p}(h W)$, and one sees immediately from the definitions that $\omega^{p}(f \circ g)=\omega^{p}(f) \circ \omega^{p}(g)$. The isomorphisms

$$
\omega^{p}(h V \otimes h W) \stackrel{\text { 50 }}{=} \omega^{p}(h(V \times W)) \stackrel{2.7}{\cong} \omega^{p}(h V) \otimes \omega^{p}(h W)
$$

provide $\omega^{p}$ with the structure of a tensor functor $\mathcal{C} \mathcal{V}^{0}(k) \rightarrow \operatorname{Mod}\left(\mathbb{A}_{f}^{p}\right)$. It is $\mathbb{Q}$-linear (in particular, additive).

Theorem 4.8. The functor $\omega^{p}: \mathcal{C} \mathcal{V}^{0}(k) \rightarrow \operatorname{Mod}\left(\mathbb{A}_{f}^{p}\right)$ extends (essentially uniquely) to a $\mathbb{Q}$-linear tensor functor $\omega^{p}: \mathcal{M}(k ; \mathbb{Q} ; \mathcal{S}) \rightarrow \operatorname{Mod}\left(\mathbb{A}_{f}^{p}\right)$. For each $\ell \neq p, \omega^{p} \otimes_{\mathbb{A}_{f}^{p}}$ $\mathbb{Q}_{\ell} \cong \omega_{\ell}$.

Proof. As $\operatorname{Mod}\left(\mathbb{A}_{f}^{p}\right)$ is abelian, $\omega^{p}$ extends (essentially uniquely) to $\mathcal{M}^{+}(k ; \mathbb{Q} ; \mathcal{S})$ because of the universality of the pseudo-abelian hull. Because $\omega^{p}(\mathbb{L})=\mathbb{A}_{f}^{p}(-1)$ is invertible in $\operatorname{Mod}\left(\mathbb{A}_{f}^{p}\right), \omega^{p}$ has an essentially unique extension from $\mathcal{M}^{+}(k ; \mathbb{Q} ; \mathcal{S})$ to $\mathcal{M}(k ; \mathbb{Q} ; \mathcal{S})$. For $V \in \mathcal{S}, \omega^{p}(h V) \otimes_{\mathbb{A}_{f}^{p}} \mathbb{Q}_{\ell} \cong \omega_{\ell}(h V)$, and this property is preserved under each extension.

Remark 4.9. The following formula holds:

$$
\omega^{p}(h(V, e, m))=e\left(H^{*}\left(\bar{V}, \mathbb{A}_{f}^{p}(m)\right)\right)
$$

where, on the right, $e$ denotes the map defined in $(2.7 \mathrm{~d})$. For a motive $X=$ $h(V, e, m)$, define $\lambda_{\ell}(X)$ to be the image of

$$
H^{*}\left(\bar{V}, \mathbb{Z}_{\ell}(m)\right) \rightarrow H^{*}\left(\bar{V}, \mathbb{Q}_{\ell}(m)\right) \stackrel{e}{\rightarrow} H^{*}\left(\bar{V}, \mathbb{Q}_{\ell}(m)\right) .
$$

Then $\omega^{p}(X)$ is the restricted direct product

$$
\omega^{p}(X)=\prod_{\ell \neq p}\left(\omega_{\ell}(X), \lambda_{\ell}(X)\right) .
$$

Proposition 4.10. Let $\sim$ be numerical equivalence, and assume that it coincides with $\ell$-adic homological equivalence on algebraic cycles with $\mathbb{Q}$-coefficients $(\ell \neq p)$ for the varieties in $\mathcal{S}$. Then, for all isomotives $X$,

(a) $\omega^{p}(X)$ is a free $\mathbb{A}_{f}^{p}$-module of finite rank;

(b) for all morphisms $\alpha$ of isomotives, $\omega^{p}(\alpha)$ has constant rank (see 2.4).

Proof. The hypotheses imply that the Künneth projectors are algebraic for $\ell \neq p$ (see 4.7), and when we use them to modify the commutativity constraints (see 4.6), the resulting category $\mathcal{M}(k ; \mathbb{Q} ; \mathcal{S})$ is Tannakian $[26$. In any Tannakian category over a field, there is a good notion of the rank of an object $X$ (defined to be the trace of the identity map on $X$; see [47, I, 5.1.4]), and for any fibre functor $\omega$ with values in the vector spaces over a field and any morphism $\alpha: X \rightarrow Y$ in the category,

$$
\begin{aligned}
\operatorname{dim}(\omega(X)) & =\operatorname{rank}(X), \\
\operatorname{rank}(\omega(\alpha)) & =\operatorname{rank} X-\operatorname{rank} \operatorname{Ker}(\alpha) .
\end{aligned}
$$

\footnotetext{
${ }^{9}$ The functor $\omega_{\ell}$ commutes with tensor products, but it will not preserve the commutativity constraints unless these have been modified as in [4.6).
} 
In particular, $\operatorname{dim}(\omega(X))$ and $\operatorname{rank}(\omega(\alpha))$ are independent of $\omega$. When we apply this statement to the functors $\omega_{\ell}$, we obtain the theorem.

The functor $\omega^{p}: \mathcal{M}(k ; \mathbb{Q} ; \mathcal{S}) \rightarrow \mathcal{R}\left(k ; \mathbb{A}_{f}^{p}\right)$.

Theorem 4.11. The functor $\omega_{\ell}$ has a canonical factorization through the forgetful functor $\mathcal{R}\left(k ; \mathbb{Q}_{\ell}\right) \rightarrow \operatorname{Vec}\left(\mathbb{Q}_{\ell}\right)$. Under the hypotheses of $\left[4.10\right.$, the functor $\omega^{p}$ has a canonical factorization through the forgetful functor $\mathcal{R}\left(k ; \mathbb{A}_{f}^{p}\right) \rightarrow \operatorname{Mod}\left(\mathbb{A}_{f}^{p}\right)$.

Proof. Let $V \in \mathcal{S}$. When $k$ is finitely generated over the prime field, there is a canonical continuous action of $\operatorname{Gal}\left(k^{\mathrm{sep}} / k\right)$ on $H^{i}\left(\bar{V}, \mathbb{Q}_{\ell}(m)\right)$, which therefore lies in $\mathcal{R}\left(k ; \mathbb{Q}_{\ell}\right)$. For any correspondence $f \in \operatorname{Corr}^{r}(V, W)$, the map

$$
f: H^{i}\left(\bar{V}, \mathbb{Q}_{\ell}(m)\right) \rightarrow H^{i+r}\left(\bar{W}, \mathbb{Q}_{\ell}(m+r)\right)
$$

respects the actions of $\operatorname{Gal}\left(k^{\mathrm{sep}} / k\right)$. Therefore, the functor

$$
\omega_{\ell}: \mathcal{M}(k ; \mathbb{Q}) \rightarrow \operatorname{Vec}\left(\mathbb{Q}_{\ell}\right)
$$

has a canonical factorization through the forgetful functor $\mathcal{R}\left(k ; \mathbb{Q}_{\ell}\right) \rightarrow \operatorname{Vec}\left(\mathbb{Q}_{\ell}\right)$. The argument for $\omega^{p}$ is similar.

For an arbitrary field $k$, any triple $(V, W, f)$ consisting of varieties $V, W \in \mathcal{S}$ and a correspondence $f$ from $V$ to $W$ will have a model $\left(V_{1}, W_{1}, f_{1}\right)$ over some subfield $k_{1}$ of $k$ finitely generated over the prime field. The image of $\omega_{\ell}\left(f_{1}\right): \omega_{\ell}\left(V_{1}\right) \rightarrow$ $\omega_{\ell}\left(W_{1}\right)$ under the functor $\mathcal{R}\left(k_{1} ; \mathbb{Q}_{\ell}\right) \rightarrow \mathcal{R}\left(k ; \mathbb{Q}_{\ell}\right)$ is independent of the choice of $k_{1}, V_{1}, W_{1}, f_{1}$. Thus, the statements hold also for $k$.

\section{Categories of (integral) motives}

We fix a field $k$ of characteristic exponent $p$, and a class $\mathcal{S}$ of smooth projective varieties over $k$ satisfying the condition (4.1) and such that

$\left(^{*}\right)$ : for all $V$ in $\mathcal{S}$ and all $l$ (including $p$ ), numerical equivalence coincides with $l$-adic homological equivalence for algebraic cycles with $\mathbb{Q}$-coefficients.

As we noted in (4.7), this condition implies that the Künneth components of the diagonal are algebraic, and so we can define $\mathcal{M}^{+}(k ; \mathbb{Q})$ and $\mathcal{M}(k ; \mathbb{Q})$ to be the categories $\mathcal{M}_{\text {num }}^{+}(k ; \mathbb{Q} ; \mathcal{S})$ and $\mathcal{M}_{\text {num }}(k ; \mathbb{Q} ; \mathcal{S})$ in $[4.6)$, i.e., with the modified commutativity constraints. Recall that they are semisimple abelian $\mathbb{Q}$-categories $[26$.

Because $\omega_{l}$ is a fibre functor on $\mathcal{M}(k ; \mathbb{Q})$, the map

$$
\operatorname{Hom}_{\mathcal{M}(k ; \mathbb{Q})}(X, Y)_{\mathbb{Q}_{l}} \rightarrow \operatorname{Hom}_{\mathcal{R}\left(k ; \mathbb{Q}_{l}\right)}\left(\omega_{l}(X), \omega_{l}(Y)\right)
$$

defined by $\omega_{l}$ is injective [8,2.13]. It follows that it is also injective when $\mathcal{M}$ and $\mathcal{R}$ are replaced by $\mathcal{M}^{+}$and $\mathcal{R}^{+}$. We say that the l-adic Tate conjecture holds for $\mathcal{M}(k ; \mathbb{Q})$ or $\mathcal{M}^{+}(k ; \mathbb{Q})$ if the corresponding map is an isomorphism for $l$.

While $\left(^{*}\right)$ is conjectured to hold for all smooth projective varieties, it has been proved for very few varieties (see 31 for a list). In the final subsection of this section (Variants, p. 521), we list some alternative categories $\mathcal{M}^{+}(k ; \mathbb{Q})$ and $\mathcal{M}(k ; \mathbb{Q})$ to which our constructions apply, but which require no unproven conjectures.

Our construction of $\mathcal{M}(k ; \mathbb{Z})$ is suggested by the following simple observation: for a finitely generated $\mathbb{Z}$-module $M, M \cong M_{\hat{\mathbb{Z}}} \times_{M_{\mathbb{A}_{f}}} M_{\mathbb{Q}}$; therefore, any category whose Hom-sets are finitely generated $\mathbb{Z}$-modules admits a fully faithful functor into the fibre product of a $\hat{\mathbb{Z}}$-category with a $\mathbb{Q}$-category over an $\mathbb{A}_{f}$-category. 
The category of effective motives $\mathcal{M}^{+}(k ; \mathbb{Z})$.

Definition 5.1. The category of effective motives $\mathcal{M}^{+}(k ; \mathbb{Z})$ over $k$ is the full subcategory of the fibre product category

$$
\mathcal{R}^{+}(k ; \hat{\mathbb{Z}}) \times_{\mathcal{R}^{+}\left(k ; \mathbb{A}_{f}\right)} \mathcal{M}^{+}(k ; \mathbb{Q})
$$

whose objects $\left(X_{f}, X_{0}, x_{f}\right)$ are those for which the prime-to- $p$ torsion subgroup of $X_{f}$ is finite. An effective motive $X=\left(X_{f}, X_{0}, x_{f}\right)$ is torsion if $X_{0}=0$.

Thus, an effective motive $X$ over $k$ is a triple $\left(X_{f}, X_{0}, x_{f}\right)$ consisting of

(a) an object $X_{f}=\left(X_{l}\right)_{l}$ of $\mathcal{R}^{+}(k ; \hat{\mathbb{Z}})$ such that $X_{l}$ is torsion-free for almost all $l$,

(b) an effective isomotive $X_{0}$, and

(c) an isomorphism $x_{f}:\left(X_{f}\right)_{\mathbb{Q}} \rightarrow \omega_{f}\left(X_{0}\right)$ in $\mathcal{R}^{+}\left(k ; \mathbb{A}_{f}\right)$.

A morphism of effective motives $\alpha: X \rightarrow Y$ is a pair of morphisms

$$
\left(\alpha_{f}: X_{f} \rightarrow Y_{f}, \alpha_{0}: X_{0} \rightarrow Y_{0}\right)
$$

such that

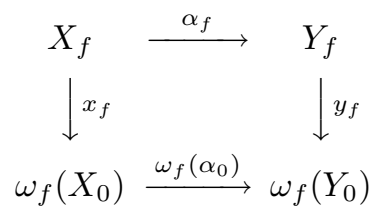

commutes.

Note that to give $\left(X_{f}, x_{f}\right)$ amounts to giving an $X_{l}$ in $\mathcal{R}\left(k ; \mathbb{Z}_{l}\right)$ for each $l$ together with homomorphisms $x_{l}: X_{l} \rightarrow \omega_{l}\left(X_{0}\right)$ that induce isomorphisms $X_{l} \rightarrow \lambda_{l}\left(X_{0}\right)$ for almost all $l$ and isomorphisms $\left(X_{l}\right)_{\mathbb{Q}} \rightarrow \omega_{l}\left(X_{0}\right)$ for all $l$. We usually regard $x_{f}$ as a map $X_{f} \rightarrow \omega_{f}\left(X_{0}\right)$ with torsion kernel. We use the notations: $X_{f}=X^{p} \times X_{p}$, $X^{p}=\prod_{l \neq p} X_{l}, x_{f}=x^{p} \times x_{p}, \alpha_{f}=\alpha^{p} \times \alpha_{p}$.

Proposition 5.2. The category $\mathcal{M}^{+}(k ; \mathbb{Z})$ is abelian. A sequence

$$
X \rightarrow Y \rightarrow Z
$$

in $\mathcal{M}^{+}(k ; \mathbb{Z})$ is exact if and only if the sequences

$$
X_{0} \rightarrow Y_{0} \rightarrow Z_{0} \text { and } X_{f} \rightarrow Y_{f} \rightarrow Z_{f}
$$

are exact in $\mathcal{M}^{+}(k ; \mathbb{Q})$ and $\mathcal{R}^{+}(k ; \hat{\mathbb{Z}})$, respectively.

Proof. Both statements are true for the fibre product category, and so it suffices to show that $\mathcal{M}^{+}(k ; \mathbb{Z})$ is closed under the formation of kernels and cokernels in the larger category. Any subobject of an object in $\mathcal{M}^{+}(k ; \mathbb{Z})$ is also in $\mathcal{M}^{+}(k ; \mathbb{Z})$. Therefore, $\mathcal{M}^{+}(k ; \mathbb{Z})$ is closed under the formation of kernels, and, in proving that the cokernel of $\alpha$ is in $\mathcal{M}^{+}(k ; \mathbb{Z})$, we may assume that $\alpha$ is injective. Thus, given a short exact sequence

$$
0 \rightarrow X \stackrel{\alpha}{\rightarrow} Y \rightarrow Z \rightarrow 0
$$

in the fibre product category with $X, Y$ in $\mathcal{M}^{+}(k ; \mathbb{Z})$, we have to show that $Z$ is in $\mathcal{M}^{+}(k ; \mathbb{Z})$. As $\mathcal{M}^{+}(k ; \mathbb{Q})$ is semisimple, the sequence of $\mathcal{M}^{+}(k ; \mathbb{Q})$-components splits. But $\omega^{p}$ is additive, and so

$$
0 \rightarrow \omega^{p}\left(X_{0}\right) \stackrel{\omega^{p}\left(\alpha_{0}\right)}{\longrightarrow} \omega^{p}\left(Y_{0}\right) \rightarrow \omega^{p}\left(Z_{0}\right) \rightarrow 0
$$


is split-exact, which implies that

$$
0 \rightarrow \lambda_{\ell}\left(X_{0}\right) \rightarrow \lambda_{\ell}\left(Y_{0}\right) \rightarrow \lambda_{\ell}\left(Z_{0}\right) \rightarrow 0
$$

is split-exact for almost all $\ell$. In turn, this implies that

$$
0 \rightarrow X_{\ell} \stackrel{\alpha_{\ell}}{\rightarrow} Y_{\ell} \rightarrow Z_{\ell} \rightarrow 0
$$

is split-exact for almost all $\ell$. Thus, the torsion subgroup of $Z_{\ell}$ is zero for almost all $\ell$ and finite for all $\ell$, which shows that $Z$ is in $\mathcal{M}^{+}(k ; \mathbb{Z})$.

Corollary 5.3. Let $X$ and $X^{\prime}$ be submotives of an effective motive $Y$, and let $X \cap X^{\prime}=X \times_{Y} X^{\prime}$. If $X_{0}=Y_{0}$, then the cokernel $Z$ of $X \cap X^{\prime} \rightarrow X^{\prime}$ is torsion.

Proof. Because $X \mapsto X_{0}$ is exact, $\left(X \times_{Y} X^{\prime} \rightarrow X^{\prime}\right)_{0} \cong\left(X_{0} \times_{Y_{0}} X_{0}^{\prime} \rightarrow X_{0}^{\prime}\right)$, which is an isomorphism if $X_{0}=Y_{0}$.

Proposition 5.4. The category $\mathcal{M}^{+}(k ; \mathbb{Z})$ is noetherian (i.e., its objects are noetherian).

Proof. Let

$$
X(1) \subset X(2) \subset X(3) \subset \cdots
$$

be a sequence of submotives of an effective motive $Y$. Eventually, the sequence $X(1)_{0} \subset X(2)_{0} \subset \cdots$ becomes stationary, equal, say, to $X(\infty)_{0} \subset Y_{0}$. Let $X(\infty)_{f}=Y_{f} \times_{\omega_{f}\left(Y_{0}\right)} \omega_{f}\left(X(\infty)_{0}\right)$. Then $X(\infty)=\left(X(\infty)_{f}, X(\infty)_{0}, \cdot\right)$ is an effective motive, and $X(1) \subset X(2) \subset \cdots$ is a sequence of submotives of $X(\infty)$ such that $X(r)_{0}=X(\infty)_{0}$ for $r \geq r_{1}$, some $r_{1}$. Now $X(\infty) / X\left(r_{1}\right)$ is torsion (5.3)), and so the sequence $X(1) \subset X(2) \subset \cdots$ becomes stationary.

Lemma 5.5. Given a morphism $\beta: X_{0} \rightarrow Y_{0}$ of effective isomotives, there exists an integer $m$ such that $m \beta$ is in the image of $\operatorname{Hom}(X, Y) \rightarrow \operatorname{Hom}\left(X_{0}, Y_{0}\right)$.

Proof. We have to find an integer $m$ and morphisms $\alpha_{l}$ such that the diagrams

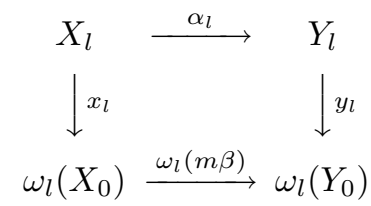

commute. Because $\omega_{l}(\beta)$ is the $l$-component of a homomorphism $\omega^{p}\left(X_{0}\right) \rightarrow \omega^{p}\left(Y_{0}\right)$, it maps $x_{l}\left(X_{l}\right)$ into $y_{l}\left(Y_{l}\right)$ for almost all $l$, and so there exists an integer $m$ such that $m \omega_{l}(\beta)$ maps $x_{l}\left(X_{l}\right)$ into $y_{l}\left(Y_{l}\right)$ for all $l$. Choose an integer $n$ that kills the torsion in $Y_{l}$ for all $l$. Then the map

$$
X_{l} \stackrel{x_{l}}{\longrightarrow} x_{l} X_{l} \stackrel{\omega_{l}(m n \beta)}{\longrightarrow} y_{l} Y_{l}=Y_{l} / \text { torsion }
$$

lifts to a map $X_{l} \rightarrow Y_{l}$.

Proposition 5.6. For all $X, Y$ in $\mathcal{M}^{+}(k ; \mathbb{Z}) \operatorname{Hom}(X, Y)$ is a finitely generated $\mathbb{Z}$-module (modulo $p$-torsion when $k$ is infinite and $p \neq 1$ ).

Proof. As $\operatorname{Hom}(X, Y) \subset \operatorname{End}(X \oplus Y)$, it suffices to show that all endomorphism rings are finitely generated $\mathbb{Z}$-modules (modulo ...). Because $\omega^{p}$ is faithful, $X \mapsto$ $X_{f}$ is faithful. In particular, the map

$$
\alpha \mapsto \alpha_{f}: \operatorname{End}(X) \rightarrow \operatorname{End}\left(X_{f}\right)
$$


is injective, and so the torsion subgroup of $\operatorname{End}(X)$ is contained in that of $\operatorname{End}\left(X_{f}\right)$, which is finite except possibly for the $p$-torsion when $k$ is infinite and $p \neq 1$. The kernel of the map

$$
\alpha \mapsto \alpha_{0}: \operatorname{End}(X) \rightarrow \operatorname{End}\left(X_{0}\right)
$$

is obviously torsion, and so it remains to show that its image $R$ is finitely generated over $\mathbb{Z}$. If $\beta \in \operatorname{End}\left(X_{0}\right)$ is in $R$, then $\omega_{l}(\beta)$ stabilizes the lattice $x_{l}\left(X_{l}\right)$ for all $l$. Thus, the characteristic polynomial of $\beta$ has coefficients in $\mathbb{Z} \subset \mathbb{Q}$. Let $\alpha_{1}, \ldots, \alpha_{n}$ be a basis for $\operatorname{End}\left(X_{0}\right)$ as a $\mathbb{Q}$-vector space. According to Lemma 5.5, after possibly replacing each $\alpha_{i}$ with $m \alpha_{i}$ for some integer $m$, we may suppose that each $\alpha_{i} \in R$. Because $\mathcal{M}^{+}(k ; \mathbb{Q})$ is semisimple, $\operatorname{End}\left(X_{0}\right)$ is a semisimple $\mathbb{Q}$-algebra, and so the trace pairing $\alpha, \beta \mapsto \operatorname{Tr}_{\operatorname{End}\left(X_{0}\right) / \mathbb{Q}}(\alpha \beta)$ is nondegenerate. Therefore, there is a $\mathbb{Q}$ basis $\beta_{1}, \ldots, \beta_{n}$ for $\operatorname{End}\left(X_{0}\right)$ dual to $\alpha_{1}, \ldots, \alpha_{n}$. Now the usual argument shows that

$$
R \subset \mathbb{Z} \beta_{1}+\cdots+\mathbb{Z} \beta_{n}
$$

Namely, let $\beta \in R$. Then $\beta$ can be written uniquely as a linear combination $\beta=\sum b_{j} \beta_{j}$ of the $\beta_{j}$ with coefficients $b_{j} \in \mathbb{Q}$, and we have to show that each $b_{j} \in \mathbb{Z}$. As $\alpha_{i}$ and $\beta$ are in $R$, so also is $\beta \cdot \alpha_{i}$, and so $\operatorname{Tr}\left(\beta \cdot \alpha_{i}\right) \in \mathbb{Z}$. But

$$
\operatorname{Tr}\left(\beta \cdot \alpha_{i}\right)=\operatorname{Tr}\left(\sum_{j} b_{j} \beta_{j} \alpha_{i}\right)=\sum_{j} b_{j} \operatorname{Tr}\left(\beta_{j} \alpha_{i}\right)=b_{i} .
$$

Remark 5.7. (a) If $Y$ has no $p$-torsion (i.e., $Y_{p}$ is torsion-free), then $\operatorname{Hom}(X, Y)$ has no $p$-torsion and hence is finitely generated over $\mathbb{Z}$.

(b) In nonzero characteristic, the effective motive $X$ with $X_{0}=0$ and $X_{f}=X_{p}=$ $k$ ( $F$ acting as zero) has $\operatorname{End}(X)=k$. The same phenomenon occurs with subgroup schemes of abelian varieties: a supersingular elliptic curve has $\alpha_{p}=\operatorname{Spec} k[T] /\left(T^{p}\right)$ as a subgroup, and $\operatorname{End}\left(\alpha_{p}\right)=k$.

Proposition 5.8. The functor $X \mapsto X_{0}: \mathcal{M}^{+}(\mathbb{F} ; \mathbb{Z}) \rightarrow \mathcal{M}^{+}(\mathbb{F} ; \mathbb{Q})$ defines an equivalence of categories

$$
\mathcal{M}^{+}(\mathbb{F} ; \mathbb{Z})_{\mathbb{Q}} \rightarrow \mathcal{M}^{+}(\mathbb{F} ; \mathbb{Q}) .
$$

Proof. Because the kernel of

$$
\alpha \mapsto \alpha_{0}: \operatorname{Hom}(X, Y) \rightarrow \operatorname{Hom}\left(X_{0}, Y_{0}\right)
$$

is torsion, the map

$$
r \otimes \alpha \mapsto r \alpha_{0}: \mathbb{Q} \otimes \operatorname{Hom}(X, Y) \rightarrow \operatorname{Hom}\left(X_{0}, Y_{0}\right)
$$

is injective, and Lemma 5.5 shows that it is surjective. Thus, the functor is faithful and full. Given an $X$ in $\mathcal{M}^{+}(k ; \mathbb{Q})$, clearly there exists an $X^{p}$ in $\mathcal{R}^{+}\left(k ; \mathbb{Z}^{p}\right)$ such that $\left(X^{p}\right)_{\mathbb{Q}} \approx \omega^{p}(X)$. Using this, one sees that $X \mapsto X_{0}$ is essentially surjective.

Remark 5.9. The torsion effective motives form a thick subcategory of $\mathcal{M}^{+}(k ; \mathbb{Z})$, and Proposition 5.8 shows that the functor

$$
X \mapsto X_{0}: \mathcal{M}^{+}(k ; \mathbb{Z}) \rightarrow \mathcal{M}^{+}(k ; \mathbb{Q})
$$

realizes $\mathcal{M}^{+}(k ; \mathbb{Q})$ as the quotient of $\mathcal{M}^{+}(k ; \mathbb{Z})$ by its subcategory of torsion objects.

Proposition 5.10. The functor

$$
\mathcal{M}^{+}(k ; \mathbb{Z})_{\mathbb{Z}_{l}} \rightarrow \mathcal{R}^{+}\left(k ; \mathbb{Z}_{l}\right)
$$

defined by $X \mapsto X_{l}$ is faithful; it is full if and only if the l-adic Tate conjecture holds for $\mathcal{M}^{+}\left(k ; \mathbb{Q}_{l}\right)$. 
Proof. We first show that

$$
z \otimes \alpha \mapsto z \alpha_{l}: \mathbb{Z}_{l} \otimes_{\mathbb{Z}} \operatorname{Hom}_{\mathcal{M}^{+}(k ; \mathbb{Z})}(X, Y) \rightarrow \operatorname{Hom}_{\mathcal{R}^{+}\left(k ; \mathbb{Z}_{l}\right)}\left(X_{l}, Y_{l}\right)
$$

is injective. The torsion subgroup of $\mathbb{Z}_{l} \otimes \operatorname{Hom}_{\mathcal{M}^{+}(k ; \mathbb{Z})}(X, Y)$ is equal to the $l$ primary component of the torsion subgroup of $\operatorname{Hom}_{\mathcal{M}}+(k ; \mathbb{Z})(X, Y)$, and this maps injectively to $\operatorname{Hom}_{\mathcal{R}^{+}\left(k ; \mathbb{Z}_{l}\right)}\left(X_{l}, Y_{l}\right)$ (because the functor $X \mapsto X_{f}$ is faithful). Thus, the kernel of $z \otimes \alpha \mapsto z \alpha_{l}$ is torsion-free. Consider the diagram

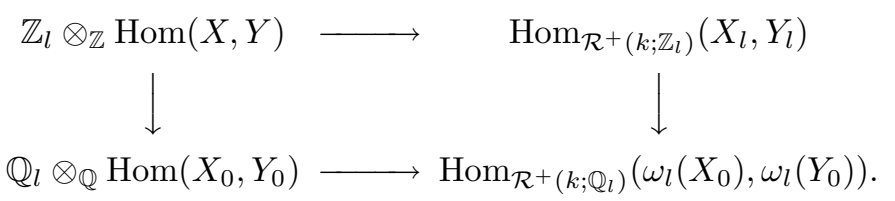

The left vertical arrow is obtained from

$$
\operatorname{Hom}(X, Y) \rightarrow \operatorname{Hom}\left(X_{0}, Y_{0}\right)
$$

by tensoring with $\mathbb{Z}_{l}$, and hence has torsion kernel (5.8). The bottom arrow is injective (6). Hence, the kernel of the top horizontal arrow is torsion, but we have already shown it to be torsion-free; it is therefore zero.

We next show that the cokernel of $z \otimes \alpha \mapsto z \alpha_{l}$ is torsion-free. Suppose that $\beta \in \operatorname{Hom}_{\mathcal{R}^{+}\left(k ; \mathbb{Z}_{l}\right)}\left(X_{l}, Y_{l}\right)$ is such that $l^{m} \beta=z \alpha_{l}$ for some $m \in \mathbb{N}, \alpha \in \operatorname{Hom}(X, Y)$, and $z \in \mathbb{Z}_{l}$. Because $z \otimes \alpha \mapsto z \alpha_{l}$ is (obviously) surjective on torsion, we may suppose that $z$ is not divisible by $l$. Then $\alpha_{l}$ is divisible by $l^{m}$ in $\operatorname{Hom}\left(X_{l}, Y_{l}\right)$. For $l^{\prime} \neq l, l^{m}$ is a unit in $\mathbb{Z}_{l^{\prime}}$, and so $\alpha_{l^{\prime}}$ is also divisible by $l^{m}$. Now $\alpha^{\prime}$, with

$$
\alpha_{0}^{\prime}=l^{-m} \alpha_{0}, \quad \alpha_{l}^{\prime}=z^{-1} \beta, \quad \alpha_{l^{\prime}}^{\prime}=l^{-m} \alpha_{l^{\prime}} \text { for } l^{\prime} \neq l
$$

is a morphism $X \rightarrow Y$ such that $z \alpha_{l}^{\prime}=\beta$.

Finally, we assume the $l$-adic Tate conjecture for $\mathcal{M}^{+}(k ; \mathbb{Q})$, and we prove that the cokernel of $z \otimes \alpha \mapsto z \alpha_{l}$ is torsion. For this, it suffices to prove that

$$
\mathbb{Q}_{l} \otimes_{\mathbb{Z}} \operatorname{Hom}(X, Y) \rightarrow \mathbb{Q} \otimes_{\mathbb{Z}} \operatorname{Hom}_{\mathcal{R}^{+}\left(k ; \mathbb{Z}_{l}\right)}\left(X_{l}, Y_{l}\right)
$$

is surjective. But this follows from the isomorphisms

$$
\mathbb{Q} \otimes_{\mathbb{Z}} \operatorname{Hom}(X, Y) \stackrel{(5.8)}{\cong} \operatorname{Hom}\left(X_{0}, Y_{0}\right)
$$

and

$\mathbb{Q}_{l} \otimes_{\mathbb{Q}} \operatorname{Hom}\left(X_{0}, Y_{0}\right) \stackrel{\text { Tate }}{\cong} \operatorname{Hom}_{\mathcal{R}^{+}\left(k ; \mathbb{Q}_{l}\right)}\left(\omega_{l}\left(X_{0}\right), \omega_{l}\left(Y_{0}\right)\right) \cong \mathbb{Q} \otimes_{\mathbb{Z}} \operatorname{Hom}_{\mathcal{R}^{+}\left(k ; \mathbb{Z}_{l}\right)}\left(X_{l}, Y_{l}\right)$.

Proposition 5.11. The category

$$
\mathcal{M}^{+}(k ; \mathbb{Z})=\underset{k^{\prime}}{\lim } \mathcal{M}^{+}\left(k^{\prime} ; \mathbb{Z}\right)
$$

where $k^{\prime}$ runs over the subfields of $k$ finitely generated over the prime field.

Proof. The statement is true for each of the categories $\mathcal{M}^{+}(k ; \mathbb{Q}), \mathcal{R}^{+}(k ; \hat{\mathbb{Z}})$, and $\mathcal{R}^{+}\left(k ; \mathbb{A}_{f}\right)$, and it follows easily for their fibre product and its subcategory $\mathcal{M}^{+}(k ; \mathbb{Z})$. 
The category of motives $\mathcal{M}(k ; \mathbb{Z})$.

Definition 5.12. The category of motives $\mathcal{M}(k ; \mathbb{Z})$ is the full subcategory of

$$
\mathcal{R}(k ; \hat{\mathbb{Z}}) \times_{\mathcal{R}\left(k ; \mathbb{A}_{f}\right)} \mathcal{M}(k ; \mathbb{Q})
$$

whose objects $\left(X_{f}, X_{0}, x_{f}\right)$ are those for which the prime-to- $p$ torsion subgroup of $X_{f}$ is finite.

The results in the previous subsection hold mutatis mutandis for $\mathcal{M}(k ; \mathbb{Z})$ - in particular, $\mathcal{M}(k ; \mathbb{Z})$ is a noetherian abelian category whose quotient by its torsion subcategory is $\mathcal{M}(k ; \mathbb{Q})$.

Proposition 5.13. The obvious functor $\mathcal{M}^{+}(k ; \mathbb{Z}) \rightarrow \mathcal{M}(k ; \mathbb{Z})$ is faithful, and it is full on torsion-free objects (on all objects when $k$ has characteristic zero). It realizes $\mathcal{M}(k ; \mathbb{Z})$ as the category obtained from $\mathcal{M}^{+}(k ; \mathbb{Z})$ by inverting $\mathbb{L}$.

Proof. Straightforward.

Remark 5.14. (a) When $k=\mathbb{Q}$ there is a canonical functor from $\mathcal{M}(k ; \mathbb{Z})$ to the category of systems of realizations over $\mathbb{Q}$ with integer coefficients defined in [7] 1.23].

(b) Let $k$ be a subfield of $\mathbb{C}$, and let $\mathcal{M}(k ; \mathbb{Q})$ be the category based on a class $\mathcal{S}$ satisfying (4.1) but using the "motivated" classes as correspondences [1, 4.2]. In this case, our definition of $\mathcal{M}(k ; \mathbb{Z})$ is equivalent to that of [1, 8.1].

The tensor structures on $\mathcal{M}^{+}(k ; \mathbb{Z})$ and $\mathcal{M}(k ; \mathbb{Z})$. Because we have modified the commutativity constraint, $\mathcal{M}(k ; \mathbb{Q}) \stackrel{\omega_{l}}{\rightarrow} \mathcal{R}_{l}\left(k ; \mathbb{Q}_{l}\right)$ and its composite with $\mathcal{M}^{+}(k ; \mathbb{Q}) \rightarrow \mathcal{M}(k ; \mathbb{Q})$ are tensor functors. For two effective motives, $X=\left(\left(X_{l}\right)\right.$, $\left.X_{0},\left(x_{l}\right)\right)$ and $Y=\left(\left(Y_{l}\right), Y_{0},\left(y_{l}\right)\right)$ we define

$$
X \otimes Y=\left(\left(X_{l} \otimes Y_{l}\right), X_{0} \otimes Y_{0},\left(x_{l} \otimes y_{l}\right)\right) .
$$

With this structure, $\mathcal{M}^{+}(k ; \mathbb{Z})$ and $\mathcal{M}(k ; \mathbb{Z})$ become abelian tensor $\mathbb{Z}$-categories. Moreover, it follows from (5.2) that $\otimes$ is right exact.

Lemma 5.15. The category $\mathcal{M}(k ; \mathbb{Z})$ has internal Homs.

Proof. The category $\mathcal{R}\left(k ; \mathbb{Z}_{l}\right)$ has internal Homs - for $l=p$ see (1.8) and for $l \neq p$ this is obvious - and the functors $\omega_{l}: \mathcal{M}(k ; \mathbb{Q}) \rightarrow \mathcal{R}\left(k ; \mathbb{Z}_{p}\right)$ preserve internal Homs [47, I, 4.3.1]. Let $X$ and $Y$ be motives over $k$. The isomorphisms $x_{l}:\left(X_{l}\right)_{\mathbb{Q}} \rightarrow$ $\omega_{l}\left(X_{0}\right)$ and $y_{l}:\left(Y_{l}\right)_{\mathbb{Q}} \rightarrow \omega_{l}\left(Y_{0}\right)$ define isomorphisms $\left(x_{l}, y_{l}\right): \underline{\operatorname{Hom}}\left(\left(X_{l}\right)_{\mathbb{Q}},\left(Y_{l}\right)_{\mathbb{Q}}\right) \rightarrow$ $\omega_{l}\left(\underline{\operatorname{Hom}}\left(X_{0}, Y_{0}\right)\right)$, and

$$
\underline{\operatorname{Hom}}(X, Y) \stackrel{\mathrm{df}}{=}\left(\left(\underline{\operatorname{Hom}}\left(X_{l}, Y_{l}\right)_{l}, \underline{\operatorname{Hom}}\left(X_{0}, Y_{0}\right),\left(x_{l}, y_{l}\right)_{l}\right)\right.
$$

is an internal Hom for $X$ and $Y$.

Artin motives. Fix a separable algebraic closure $\bar{k}$ of $k$, and let $\Gamma=\operatorname{Gal}(\bar{k} / k)$. The category of Artin isomotives is the full subcategory $\mathcal{M}^{\operatorname{Artin}}(k ; \mathbb{Q})$ of $\mathcal{M}^{+}(k ; \mathbb{Q})$ of objects of the form $X=h V$ with $V$ a variety of dimension 0 . For such an $X$, define $\pi_{0}(X)=\pi_{0}(\bar{V})$ - it is a finite set with a continuous action of $\Gamma$. The functor $H: X \mapsto \mathbb{Q}^{\pi_{0}(X)}={ }_{\mathrm{df}} \operatorname{Map}\left(\pi_{0}(X), \mathbb{Q}\right)$ defines an equivalence of $\mathcal{M}^{\operatorname{Artin}}(k ; \mathbb{Q})$ with the category $\mathcal{R}(\Gamma ; \mathbb{Q})$ of continuous representations of $\Gamma$ on finite-dimensional $\mathbb{Q}$-vector spaces (cf. [10, 6.17]). Hence, $\mathcal{M}^{\operatorname{Artin}}(k ; \mathbb{Q})$ is a Tannakian category. 
We define the category of Artin motives to be the full subcategory $\mathcal{M}^{\operatorname{Artin}}(k ; \mathbb{Z})$ of $\mathcal{M}^{+}(k ; \mathbb{Z})$ of objects $X$ such that $X_{0}$ is an Artin isomotive and (when $p \neq 1$ ) $F_{X_{p}}$ is bijective.

Recall (47, VI, 3.1.2]; [11 p. 69]) that there is a fully faithful functor

$$
\gamma: \mathcal{R}\left(\Gamma ; \mathbb{Z}_{p}\right) \rightarrow \operatorname{Crys}^{+}\left(k^{\mathrm{pf}}\right)=\mathcal{R}^{+}\left(k ; \mathbb{Z}_{p}\right)
$$

whose essential image consists of the crystals with bijective $F$. Let $X$ be an Artin motive. When $p \neq 1$, we choose $X_{p}^{\prime} \in \mathcal{R}\left(\Gamma ; \mathbb{Z}_{p}\right)$ so that $\gamma\left(X_{p}^{\prime}\right)=X_{p}$, and we let $\omega_{p}^{\prime}\left(X_{0}\right)=\left(X_{p}^{\prime}\right)_{\mathbb{Q}}$; otherwise we set $X_{p}^{\prime}=0=\omega_{p}^{\prime}\left(X_{0}\right)$. Define $H(X)$ so that the right-hand square in the following diagram is cartesian:

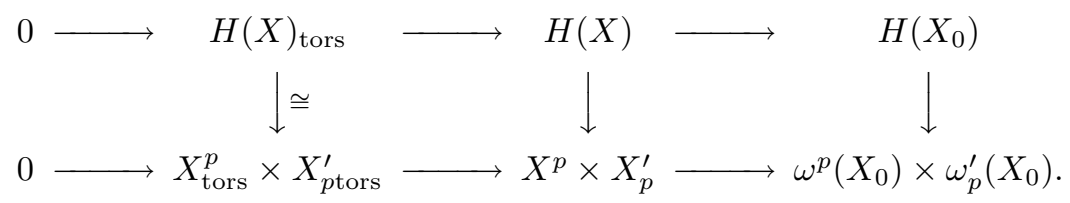

The diagram is exact and commutative, and $H(X)$ is a finitely generated $\mathbb{Z}$-module with a continuous action of $\Gamma$ (discrete topology on $H(X)$ ).

Proposition 5.16. The functor $H: \mathcal{M}^{\operatorname{Artin}}(k ; \mathbb{Z}) \rightarrow \mathcal{R}(\Gamma ; \mathbb{Z})$ is an equivalence of tensor categories.

Proof. The functor $H: \mathcal{M}^{\operatorname{Artin}}(k ; \mathbb{Q}) \rightarrow \mathcal{R}(\Gamma ; \mathbb{Q})$ defines an equivalence of categories

$$
\mathcal{R}(\Gamma ; \mathbb{Z}) \times_{\mathcal{R}(\Gamma ; \mathbb{Q})} \mathcal{M}^{\operatorname{Artin}}(k ; \mathbb{Q}) \rightarrow \mathcal{R}(\Gamma ; \mathbb{Z}) \times_{\mathcal{R}(\Gamma ; \mathbb{Q})} \mathcal{R}(\Gamma ; \mathbb{Q})=\mathcal{R}(\Gamma ; \mathbb{Z}),
$$

and $\gamma$ defines a full faithful functor

$$
\mathcal{R}(\Gamma ; \mathbb{Z}) \times_{\mathcal{R}(\Gamma ; \mathbb{Q})} \mathcal{M}^{\operatorname{Artin}}(k ; \mathbb{Q}) \rightarrow \mathcal{R}(k ; \hat{\mathbb{Z}}) \times_{\mathcal{R}\left(k ; \mathbb{A}_{f}\right)} \mathcal{M}^{\operatorname{Artin}}(k ; \mathbb{Q})
$$

whose essential image is $\mathcal{M}^{\operatorname{Artin}}(k ; \mathbb{Z})$. It is clear that the tensor products correspond.

Rationally decomposed one-motives. Recall that a one-motive is a triple $(G, X, u)$ with $G$ a semi-abelian variety, $X$ a finitely generated free $\mathbb{Z}$-module on which $\Gamma$ acts continuously, and $u$ a $\Gamma$-homomorphism $X \rightarrow G\left(k^{\text {sep }}\right)$. A one-motive $X \stackrel{u}{\rightarrow} G$ is said to be decomposed if $u=0$ and the extension

$$
0 \rightarrow T \rightarrow G \rightarrow A \rightarrow 0 \quad \text { ( } T \text { a torus, } A \text { an abelian variety) }
$$

splits, and it is rationally decomposed if it is isogenous to a decomposed one-motive. Over a finite field, every one-motive is rationally decomposed. The one-motives form an additive category $\mathcal{M}_{1}(k)$ with the decomposed and rationally decomposed one-motives as additive subcategories $\mathcal{M}_{1}^{\mathrm{d}}(k) \subset \mathcal{M}_{1}^{\text {rd }}(k) \subset \mathcal{M}_{1}(k)$.

Choose a quasi-inverse $F: \mathcal{R}(\Gamma ; \mathbb{Z}) \rightarrow \mathcal{M}^{\operatorname{Artin}}(k ; \mathbb{Z})$ to the functor $H$ of (5.16) .

Proposition 5.17. For $L=(X \stackrel{0}{\rightarrow} T \times A)$ in $\mathcal{M}_{1}^{d}(k)$, define $h L$ to be the effective motive $F(X) \oplus h_{\mathbb{Z}}^{1} A \oplus F\left(X_{*}(T)(1)\right)$. Then $h$ extends to a functor $h: \mathcal{M}_{1}^{\text {rd }}(k) \rightarrow$ $\mathcal{M}^{+}(k ; \mathbb{Z})$. (Here $h_{\mathbb{Z}}^{1} A$ denotes $h^{1} A$ endowed with the $\mathbb{Z}$-structure provided by the maps $H^{1}\left(A, \mathbb{Z}_{l}\right) \rightarrow H^{1}\left(A, \mathbb{Q}_{l}\right)$.)

Proof. Let $L=(u: X \rightarrow G)$ be a rationally decomposed one-motive, and choose an isogeny $\alpha: L \rightarrow L^{\prime}$ from $L$ to a decomposed one-motive $L^{\prime}$. For definiteness, form $L^{\prime}$ by taking the quotient $G / u X$ and then pushing out the extension $0 \rightarrow$ $T_{1} \rightarrow G / u X \rightarrow A_{1} \rightarrow 0$ by $n: T_{1} \rightarrow T_{1}$ where $n$ is the order of the extension in 
$\operatorname{Ext}^{1}\left(A_{1}, T_{1}\right)$. Then $\alpha$ induces isomorphisms $\omega^{p}(L) \cong \omega^{p}\left(L^{\prime}\right)$ and $\omega_{p}(L) \cong \omega_{p}\left(L^{\prime}\right)$. We define $h L$ to be the isomotive $\left(h L^{\prime}\right)_{0}$ equipped with the structures

$$
\begin{aligned}
& \lambda^{p} L \rightarrow \omega^{p}(L) \cong \omega^{p}\left(L^{\prime}\right) \cong \omega^{p}\left(\left(h L^{\prime}\right)_{0}\right), \\
& \lambda_{p} L \rightarrow \omega_{p}(L) \cong \omega_{p}\left(L^{\prime}\right) \cong \omega_{p}\left(\left(h L^{\prime}\right)_{0}\right) .
\end{aligned}
$$

Then $h$ can be made into a functor in an obvious way.

Proposition 5.18. The functor $h: \mathcal{M}_{1}^{\text {rd }}(k) \rightarrow \mathcal{M}^{+}(k ; \mathbb{Z})$ is fully faithful (except possibly when $k$ has characteristic 2 and is not algebraic over $\left.\mathbb{F}_{2}\right)$.

Proof. It suffices to prove this in the case that $k$ is finitely generated over the prime field, in which case it is a consequence of the following theorem of Tate [52], Zarhin 58], Faltings [14, and de Jong [29: for abelian varieties $A$ and $B$ over $k$, the map $\operatorname{Hom}_{k}(A, B)_{\mathbb{Z}_{l}} \rightarrow \operatorname{Hom}_{\mathcal{R}\left(k ; \mathbb{Z}_{l}\right)}\left(T_{l} A, T_{l} B\right)$ is bijective (except possibly when $k$ has characteristic $2 \ldots)$.

Remark 5.19. Propositions 5.17 and 5.18 show that, for any two rationally decomposed one-motives $L, M$, there is an injective homomorphism

$$
\operatorname{Ext}_{\mathcal{M}_{1}(k)}^{1}(L, M) \rightarrow \operatorname{Ext}_{\mathcal{M}^{+}(k ; \mathbb{Z})}^{1}(h L, h M) .
$$

This map will not in general be surjective because an extension of two $W[F]$ modules for which there exist maps $V$ with $F V=p=V F$ need not be similarly endowed. In fact, the formula [36. Theorem 3] for the order of the $\operatorname{Ext}^{1}$ of abelian varieties in the category of abelian varieties differs from the formula for the order computed in the category of effective motives in Theorem 10.1 below.

The categories $\mathcal{M}\left(\mathbb{F}_{q} ; \mathbb{Z}\right)$. Classes $\mathcal{S}(k)$ attached to each $k \subset \mathbb{F}$ are compatible if

(a) for a finite extension $k \subset k^{\prime}$, the functor $V \mapsto V_{/ k^{\prime}}$ sends $\mathcal{S}(k)$ into $\mathcal{S}\left(k^{\prime}\right)$, and the functor $\left(V \rightarrow \operatorname{Spec} k^{\prime}\right) \mapsto\left(V \rightarrow \operatorname{Spec} k^{\prime} \rightarrow \operatorname{Spec} k\right)$ sends $\mathcal{S}\left(k^{\prime}\right)$ into $\mathcal{S}(k)$

(b) for any $k \subset \mathbb{F}$, every $V \in \mathcal{S}(k)$ has a model in $\mathcal{S}\left(k_{0}\right)$ for some finite $k_{0} \subset k$.

For a motive $X=h(V, e, m)$ over $\mathbb{F}_{q}$, the Frobenius endomorphism of $V / \mathbb{F}_{q}$ defines a canonical Frobenius element $\pi_{X} \in \operatorname{End}(X)$. For a motive $X$ over $\mathbb{F}$, each model $X_{1} / \mathbb{F}_{p^{n}}$ of $X$ defines a pair $(\pi, n)$ with $\pi$ equal to $\pi_{X_{1}}$ regarded as an endomorphism of $X$. Any two pairs $(\pi, n),\left(\pi^{\prime}, n^{\prime}\right)$ arising in this way are equivalent in the sense that $(\pi)^{n^{\prime} m}=\left(\pi^{\prime}\right)^{n m}$ for some integer $m>0$. The germ of a Frobenius endomorphism of $X$ is the equivalence class of pairs containing the pair arising from one (hence, every) model of $X$ over a finite field.

Proposition 5.20. Assume the Tate conjecture holds for $\mathcal{M}(\mathbb{F} ; \mathbb{Q})$. Then

$$
X \mapsto X_{/ \mathbb{F}}: \mathcal{M}\left(\mathbb{F}_{p^{n}} ; \mathbb{Z}\right) \rightarrow \mathcal{M}(\mathbb{F} ; \mathbb{Z})
$$

defines an equivalence of $\mathcal{M}\left(\mathbb{F}_{p^{n}} ; \mathbb{Z}\right)$ with the category of pairs $(X, \pi)$ consisting of a motive $X$ in $\mathcal{M}(\mathbb{F} ; \mathbb{Z})$ and an endomorphism $\pi$ of $X$ such that $(\pi, n)$ represents $\pi_{X}$. A similar statement holds for effective motives.

Proof. Easy consequence of the similar statement for isomotives [40, 3.5]. 


\section{Remarks on the definition of $\mathcal{M}(k ; \mathbb{Z})$.}

5.21. As André notes [1, 8.1], in view of the counterexamples of Atiyah and Hirzebruch [3] to the original Hodge conjecture, it would be "peu judicieux" to define a category of integral motives by using algebraic cycles with integer coefficients in Grothendieck's construction, in other words, with

$$
\operatorname{Hom}(\mathbb{1}, h V(r))=Z_{\text {num }}^{r}(V) .
$$

There is also the difficulty of knowing what to replace the pseudo-abelian hull with.

With our definitions, a smooth projective variety $V$ defines a complex of motives, rather than a motive (see an article in preparation), but we can make the ad hoc definition: $h_{\mathbb{Z}} V=\bigoplus_{r} h_{\mathbb{Z}}^{r} V$ where $h_{\mathbb{Z}}^{r} V$ is the isomotive $h^{r} V$ with the $\mathbb{Z}$-structure provided by the maps $H^{r}\left(V, \mathbb{Z}_{l}\right) \rightarrow H^{r}\left(V, \mathbb{Q}_{l}\right)$. If $V$ has torsion-free cohomology, then

$$
\left.\operatorname{Hom}(\mathbb{1}, h V(r))=Z_{\text {num }}^{r}(V)_{\mathbb{Q}} \cap \prod_{l} H^{2 r}\left(V, \mathbb{Z}_{l}(r)\right)\right) .
$$

There is a map from $Z_{\text {num }}^{r}(V)$ to this group, but it will not always be onto (p. 510).

5.22. J.-M. Fontaine has pointed out to us that there is an incompatibility between our definitions in characteristic 0 and in characteristic $p$. In the first case, we use the étale cohomology to define the integral structure at every prime, whereas in characteristic $p$ we use the crystalline cohomology at the prime $p$. However, the crystalline cohomology in characteristic $p$ corresponds to the de Rham cohomology in characteristic zero, and the integral structures on the de Rham cohomology and the $p$-adic étale cohomology do not correspond. For example, in the case of good reduction, it is known that the isomorphism

$$
B_{\mathrm{dR}} \otimes_{K} H_{\mathrm{dR}}^{*}(X / K) \cong B_{\mathrm{dR}} \otimes_{\mathbb{Q}_{p}} H^{*}\left(X_{\bar{K}}, \mathbb{Q}_{p}\right)
$$

(e.g., 16, 3.2.2]) does not respect the integral structures. We do not know how to resolve this problem (or even whether it is resolvable).

Variants. We list some alternative categories $\mathcal{M}(k ; \mathbb{Q})$ to which our construction applies.

5.23. Let $\mathcal{S}$ consist of the smooth projective varieties over $k$ for which the Künneth projectors are algebraic. This class satisfies the condition 4.1, includes all abelian varieties and surfaces, and includes all smooth projective varieties when $k$ is finite. Let $\mathcal{M}_{l}(k ; \mathbb{Q})$ and $\mathcal{M}(k ; \mathbb{Q})$ be the categories of motives based on $\mathcal{S}$ using respectively the algebraic classes modulo $l$-adic homological equivalence and numerical equivalence as the correspondences. There is a tensor functor

$$
\mathcal{M}_{l}(k) \rightarrow \mathcal{M}(k)
$$

which, according to [2], has a tensor section $s_{l}$. Therefore, $\omega_{l} \circ s_{l}$ is a fibre functor on $\mathcal{M}(k ; \mathbb{Q})$. Moreover, $s_{l}$ can be chosen so that $\left(\omega_{l} \circ s_{l}\right)(h V)=H^{*}\left(V, \mathbb{Q}_{l}\right)$. If, as seems likely, $s_{l}$ can be chosen10 10 that the action of $Z_{\text {num }}^{\operatorname{dim} V}(V \times V) \subset \operatorname{End}(h V)$ on $\left(\omega_{l} \circ s_{l}\right)(h V)$ preserves the image of $H^{*}\left(V, \mathbb{Z}_{l}\right)$ in $H^{*}\left(V, \mathbb{Q}_{l}\right)$ for all $V$ with torsionfree cohomology, then the $\omega_{l} \circ s_{l}$ for $l \neq p$ are the $l$-components of an $\mathbb{A}_{f}^{p}$-valued fibre functor on $\mathcal{M}(k ; \mathbb{Q})$, and we can construct $\mathcal{M}^{+}(k ; \mathbb{Z})$ and $\mathcal{M}(k ; \mathbb{Z})$ as above.

\footnotetext{
${ }^{10}$ According to André (email of March 25, 2002) it does not appear possible to prove this by the methods of their paper.
} 
5.24. Let $\mathcal{S}$ consist of all smooth projective varieties over $k$. For any $V$ in $\mathcal{S}$, the Künneth projectors are almost algebraic (in the sense of [55, p. 76]). Therefore, the discussion in $(5.23)$ applies to the category $\mathcal{M}(k ; \mathbb{Q})$ defined using numerical equivalence classes of almost algebraic classes rather than algebraic classes.

5.25. Recall that a Lefschetz class modulo $\sim$ on a variety $V$ is an element of the $\mathbb{Q}$-subalgebra of $\oplus Z_{\sim}^{r}(V)$ generated by divisor classes. Let $\mathcal{S}$ be the smallest class of varieties over $k$ satisfying (4.1) and including the abelian varieties. Then it is possible to define a category $\mathcal{M}(k ; \mathbb{Q})$ of isomotives based on $\mathcal{S}$ using the Lefschetz classes modulo numerical equivalence as correspondences; moreover, $\mathcal{M}(k ; \mathbb{Q})$ is a semisimple Tannakian category over $\mathbb{Q}$, and there exist canonical functors $\omega_{l}$, 41 . $\S 1]$.

5.26. For any field $k$ of characteristic zero, define $\mathcal{M}(k ; \mathbb{Q})$ to be the category of motives based on some class $\mathcal{S}$ satisfying (4.1) and using the absolute Hodge classes as correspondences [10, $\S 6]$.

5.27. It is possible to construct abstractly Tannakian categories endowed with adèlic and $p$-adic fibre functors having most of the properties conjectured of $\mathcal{M}_{\text {num }}(\mathbb{F} ; \mathbb{Q})$ including the Tate conjecture. For example, let $\left(\mathrm{P}, \omega^{p}, \omega_{p}, \omega_{\infty}\right)$ be a system as in $6.2,6.3$ of $[42$. Thus, $\mathrm{P}$ is a $P$-gerbe over $\operatorname{Spec}(\mathbb{Q})$ where $P$ is the Weil-number torus. Then the category of representations of $\mathrm{P}$ is a semisimple Tannakian $\mathbb{Q}$-category with fibre functors $\omega^{p}$ and $\omega_{p}$, and it can be used to build categories $\mathcal{M}^{+}(\mathbb{F} ; \mathbb{Z})$ and $\mathcal{M}(\mathbb{F} ; \mathbb{Z})$. There exist exact tensor functors from the categories in (5.25) to these categories, and so an abelian variety over $\mathbb{F}$ defines objects in $\mathcal{M}^{+}(\mathbb{F} ; \mathbb{Z})$ and $\mathcal{M}(\mathbb{F} ; \mathbb{Z})$.

\section{TANNAKIAN PROPERTIES}

In Saavedra's book [4], Tannakian categories are defined only over a base field. We adapt his definition [47, III, 1.1.1] of an ind-Tannakian category to introduce the notion of a Tannakian category over an arbitrary noetherian ring. Throughout this section, $R$ is such a ring.

Some linear algebra. Let $\mathcal{C}$ be an $R$-linear category, and let $R^{\prime}$ be a commutative $R$-algebra. When $\mathcal{C}$ has arbitrary direct limits, Saavedra [47, I, 1.5] constructs an $R^{\prime}$-linear category $\mathcal{C}_{\left(R^{\prime}\right)}$ by "extension of the base ring". We adapt his construction to the case that $R^{\prime}$ is a finite $R$-algebra and $\mathcal{C}$ has finite direct limits (for example, $\mathcal{C}$ is abelian).

Thus, let $\mathcal{C}$ be an abelian $R$-linear category. Let $R^{\prime}$ be a finite $R$-algebra, and define $\mathcal{C}_{\left(R^{\prime}\right)}$ to be the category whose objects are pairs $\left(X, i_{X}\right)$ comprising an object $X$ of $\mathcal{C}$ and a homomorphism $i_{X}: R^{\prime} \rightarrow \operatorname{End}_{R}(X)$ of $R$-algebras [47, II, 1.5].

6.1. For any finitely generated $R$-module $M$, there is a functor (external tensor product)

$$
X \mapsto M \otimes_{R} X: \mathcal{C} \rightarrow \mathcal{C}
$$

and an isomorphism (natural in $X$ and $Y$ )

$$
\operatorname{Hom}_{\mathcal{C}}\left(M \otimes_{R} X, Y\right) \rightarrow \operatorname{Hom}_{R}\left(M, \operatorname{Hom}_{\mathcal{C}}(X, Y)\right) .
$$

For $M=R^{n}$, set $M \otimes_{R} X=X^{n}$, and extend this definition to an arbitrary $M$ by choosing a free left resolution of $M$ (cf. [47, II, 1.5.1.1]). 
6.2. The category $\mathcal{C}_{\left(R^{\prime}\right)}$ is an abelian $R^{\prime}$-linear category. A sequence

$$
\left(X^{\prime}, i_{X^{\prime}}\right) \rightarrow\left(X, i_{X}\right) \rightarrow\left(X^{\prime \prime}, i_{X^{\prime \prime}}\right)
$$

in $\mathcal{C}_{\left(R^{\prime}\right)}$ is exact if and only if

$$
X^{\prime} \rightarrow X \rightarrow X^{\prime \prime}
$$

is exact in $\mathcal{C}$. The functor $j^{R^{\prime} / R}: \mathcal{C} \rightarrow \mathcal{C}_{\left(R^{\prime}\right)}$ sending $X$ to $R^{\prime} \otimes_{R} X$ (with its canonical $R^{\prime}$-structure) is left adjoint to the functor $j_{R^{\prime} / R}: \mathcal{C}_{\left(R^{\prime}\right)} \rightarrow \mathcal{C}$ sending $\left(X, i_{X}\right)$ to $X$ [47, II, 1.5.2]. For any $X, Y \in \mathrm{ob}(\mathcal{C})$,

$$
\operatorname{Hom}_{\mathcal{C}_{\left(R^{\prime}\right)}}\left(j^{R^{\prime} / R} X, j^{R^{\prime} / R} Y\right) \cong \operatorname{Hom}_{\mathcal{C}}(X, Y) \otimes_{R} R^{\prime},
$$

and so $j^{R^{\prime} / R}$ realizes $\mathcal{C}_{R^{\prime}}$ as a full subcategory of $\mathcal{C}_{\left(R^{\prime}\right)}$.

6.3. When $R^{\prime}$ is a flat $R$-algebra, the functor $j^{R^{\prime} / R}: \mathcal{C} \rightarrow \mathcal{C}_{\left(R^{\prime}\right)}$ is exact.

6.4. Let $\mathcal{C}$ be an abelian tensor category over $R$ such that $\otimes$ is right exact. The category $\mathcal{C}_{\left(R^{\prime}\right)}$ has a canonical structure of a tensor category over $R^{\prime}$ for which $j^{R^{\prime} / R}$ and $j_{R^{\prime} / R}$ are tensor functors; moreover, $\otimes$ on $\mathcal{C}_{\left(R^{\prime}\right)}$ is right exact [47, II, 1.5.4]. Let $\mathcal{D}$ be an abelian tensor category over $R^{\prime}$ for which $\otimes$ is right exact. The map $u \mapsto u \circ j^{R^{\prime} / R}$ defines an equivalence of the category of right exact $R^{\prime}$-linear tensor functors $\mathcal{C}_{\left(R^{\prime}\right)} \rightarrow \mathcal{D}$ with the category of right exact $R$-linear tensor functors $\mathcal{C} \rightarrow \mathcal{D}$. When $R^{\prime}$ is a flat $R$-algebra, $u \mapsto u \circ j^{R^{\prime} / R}$ carries exact functors to exact functors [47] I, 1.5.3, 1.5.4]. (But it is not known that $u \circ j^{R^{\prime} / R}$ exact implies $u$ exact, even when $R^{\prime}$ is faithfully flat over $R$, except in the case that $k$ and $k^{\prime}$ are fields, when the proof requires the main theorem of 8 .)

Definitions. Let $\mathcal{C}$ be an abelian tensor $R$-category $\mathcal{C}$. Recall [47] II, 4.3.1] that an object $X$ is a $\otimes$-generator of $\mathcal{C}$ if every object of $\mathcal{C}$ is a subquotient of a direct sum of objects $X^{\otimes m}$. This definition extends easily to families of objects, and it is clear that $\mathcal{C}$ admits a $\otimes$-generator if it admits a finite family of $\otimes$-generators.

Definition 6.5. (a) A neutral Tannakian category over $R$ is a tensor category over $R$ that is tensor equivalent to $\operatorname{Rep}(G ; R)$ for some flat affine group scheme $G$ over $R$.

(b) An algebraic Tannakian category over $R$ is an abelian tensor category $\mathcal{C}$ over $R$ such that

(i) $\mathcal{C}$ has internal Homs (in the sense of [47, I, 3.1.1]),

(ii) $\mathcal{C}$ admits a $\otimes$-generator,

(iii) for some faithfully flat finite commutative $R$-algebra $R^{\prime}, \mathcal{C}_{\left(R^{\prime}\right)}$ is a neutral Tannakian category.

(c) A Tannakian category over $R$ is an abelian tensor category $\mathcal{C}$ over $R$ that is a filtered union of algebraic Tannakian categories in which the inclusions are exact tensor functors.

Let $\mathcal{C}$ and $R^{\prime}$ be as in (b), and choose an $R^{\prime}$-valued fibre functor $\omega$ on $\mathcal{C}_{\left(R^{\prime}\right)}$. Then

$$
\operatorname{Hom}_{\mathcal{C}}(X, Y) \otimes_{R} R^{\prime} \stackrel{\text { 99 }}{\cong} \operatorname{Hom}_{\mathcal{C}_{\left(R^{\prime}\right)}}\left(j^{R^{\prime} / R} X, j^{R^{\prime} / R} Y\right) \subset \operatorname{Hom}_{R^{\prime}}(\omega(X), \omega(Y)),
$$

and so $\operatorname{Hom}_{\mathcal{C}}(X, Y)$ is a finitely generated $R$-module. Thus, the Homs in any Tannakian category over $R$ are finitely generated $R$-modules. 
Remark 6.6. In the case that $R$ is a field $k$, we compare our definitions to those in [47] (corrected by the addition of the condition that $\operatorname{End}(\mathbb{1})=k-$ see $[8$; [10] $3.15])$.

(a) A tensor category over $k$ satisfies (6.5) if and only if it is a neutral Tannakian category in the sense of [47, III, 3.2.1]. This follows from a basic theorem in the theory (e.g., [10 2.11, 2.19]).

(b) A tensor category over $k$ satisfies (6.5 b) if and only if it is an algebraic Tannakian category in the sense of [47, III, 3.3.1]. This follows easily from ibid., III, 3.3 (see also [8, 6.20]).

(c) A Tannakian category over $k$ in the sense of [47, III, 3.2.1] is obviously a filtered union of algebraic Tannakian subcategories, and so satisfies (6.5) . Conversely, a category satisfying (6.5c) lacks only a fibre functor to be Tannakian in the sense of Saavedra, but such a functor can be shown to exist using Zorn's lemma (or, in characteristic zero, by applying [8, 7.1]).

A criterion to be a Tannakian category over a Dedekind domain. Let $\mathcal{C}$ be an abelian tensor category over a noetherian ring $R$. When internal Homs exist in $\mathcal{C}$, we let $X^{\vee}=\underline{\operatorname{Hom}}(X, \mathbb{1})$. There is a canonical morphism $X \rightarrow X^{\vee \vee}$, and $X$ is said to be reflexive when this morphism is an isomorphism.

Now let $R$ be a Dedekind domain. When $G$ is an affine group scheme over $R$, an object $X$ of $\operatorname{Rep}(G ; R)$ is reflexive if and only if its underlying $R$-module is projective. Serre [49, 2.2] proves that every object in $\operatorname{Rep}(G ; R)$ is a quotient of a representation of $G$ on a projective $R$-module. Thus every object in $\operatorname{Rep}(G ; R)$ is a quotient of a reflexive object. This property can be used to characterize Tannakian categories over Dedekind domains.

Proposition 6.7. Let $\mathcal{C}$ be an abelian tensor category over a Dedekind domain $R$ such that

(a) $\otimes$ is right exact,

(b) internal Homs exist, and

(c) every object of $\mathcal{C}$ is a quotient of a reflexive object.

If there exists an $R^{\prime}$-valued fibre functor on $\mathcal{C}_{\left(R^{\prime}\right)}$ for some Dedekind domain $R^{\prime}$ finite and flat over $R$, then $\mathcal{C}$ is Tannakian.

Proof. Let $\omega: \mathcal{C}_{\left(R^{\prime}\right)} \rightarrow \operatorname{Mod}\left(R^{\prime}\right)$ be such a fibre functor. To prove that $\mathcal{C}$ is Tannakian, we shall show that the functor $\underline{\operatorname{Aut}}^{\otimes}(\omega)$ of $R^{\prime}$-algebras is representable by a flat affine group scheme $G$ over $R^{\prime}$, and that $\omega$ defines an equivalence of $R^{\prime}$-tensor categories $\mathcal{C}_{\left(R^{\prime}\right)} \rightarrow \operatorname{Rep}\left(G ; R^{\prime}\right)$.

When $R^{\prime}=R$, this is proved in [47, II, 4.1]. In detail: [47, II, 4.1.1] shows that $\underline{\operatorname{End}}^{\otimes}(\omega)$ is represented by a flat affine monoid scheme $G$ over $R$ and that $\omega$ defines an equivalence of $R$-linear tensor categories $\mathcal{C} \rightarrow \operatorname{Rep}(G ; R)$, but $\underline{\text { End }}^{\otimes}(\omega) \cong$ End ${ }^{\otimes}\left(\omega_{0}\right)$ where $\omega_{0}$ is the restriction of $\omega$ to the full subcategory of reflexive objects; this last category is rigid, and so every endomorphism of $\omega_{0}$ is an automorphism [47, I, 5.2.3], which implies that $G$ is a group scheme.

According to (6.2) and (6.4), $\mathcal{C}_{\left(R^{\prime}\right)}$ is an abelian tensor category over $R^{\prime}$. Thus, the general case will follow from the neutral case once we show that $\mathcal{C}_{\left(R^{\prime}\right)}$ inherits the property that every object is a quotient of a reflexive object. Let $\left(Y, i_{Y}\right)$ be an object of $\mathcal{C}_{\left(R^{\prime}\right)}$, and let $q: X \rightarrow Y$ express $Y$ as a quotient of a reflexive object. The 
composite

$$
j^{R^{\prime} / R} X \stackrel{j^{R^{\prime} / R}(q)}{\longrightarrow} j^{R^{\prime} / R} Y \rightarrow\left(Y, i_{Y}\right)
$$

is again surjective, and $j^{R^{\prime} / R} X$ is reflexive because $X$ is.

Example 6.8. For $\ell \neq p$, the category $\mathcal{R}\left(k ; \mathbb{Z}_{\ell}\right)$ is Tannakian over $\mathbb{Z}_{\ell}$ (apply 1.5).

Example 6.9. (a) Let $k$ be a finite field, and let $\omega$ be the forgetful functor $\mathcal{R}\left(k ; \mathbb{Z}_{p}\right) \rightarrow \operatorname{Mod}(W(k))$. For $\left(X, i_{X}\right)$ in $\mathcal{R}\left(k ; \mathbb{Z}_{p}\right)_{(W(k))}$, there are two actions of $W(k)$ on $\omega(X)$, namely, that coming from the action $i_{X}$ of $W(k)$ on $X$ and that coming from the action of $W(k)$ on $\omega$, and we define $\omega^{\prime}\left(X, i_{X}\right)=W(k) \otimes_{W(k) \otimes_{\mathbb{Z}_{p}} W(k)}$ $\omega(X)$. Then $\omega^{\prime}$ is exact. Indeed, $W(k) \otimes_{\mathbb{Z}_{p}} W(k)$ is isomorphic to a product of copies of $W(k)$ indexed by the elements of $\operatorname{Gal}\left(k / \mathbb{F}_{p}\right)$, and the map $W(k) \otimes_{\mathbb{Z}_{p}} W(k) \rightarrow$ $W(k)$ corresponds to the projection to the "id"-component. Therefore, $\omega^{\prime}$ is a $W(k)$-valued fibre functor on $\mathcal{R}\left(k ; \mathbb{Z}_{p}\right)_{(W(k))}$, and $\mathcal{R}\left(k ; \mathbb{Z}_{p}\right)$ is Tannakian (apply $1.8(\mathrm{~b}))$.

(b) When $k$ has nonzero characteristic $p$ and is infinite, then $\mathcal{R}\left(k ; \mathbb{Z}_{p}\right)$ is not Tannakian (at least according to our definition, which may be too strict), because there exist objects $X$ for which $\operatorname{End}(X)$ is not a finitely generated $\mathbb{Z}_{p}$-module.

Existence of fibre functors. We refer to [7, §6] for the notion of the fundamental group of a Tannakian category $\mathcal{T}$ : it is an affine group scheme $\pi(\mathcal{T})$ in Ind $\mathcal{T}$ acting on the objects of $\mathcal{T}$; each fibre functor $\omega$ carries $\pi(\mathcal{T})$ to $\underline{\text { Aut }}^{\otimes}(\omega)$ and the action of $\pi(\mathcal{T})$ on an object $X$ to the action of $\underline{A u t}^{\otimes}(\omega)$ to $\omega(X)$. The Tannakian category $\mathcal{T}$ is algebraic if and only if $\pi(\mathcal{T})$ is algebraic (i.e., has algebraic realizations; see [47] 3.3]. When $\pi(\mathcal{T})$ is commutative, it lies in Ind $\mathcal{T}^{0}$ where $\mathcal{T}^{0}$ is the full subcategory of trivial objects. Since $\operatorname{Hom}(\mathbb{1},-): \mathcal{T}^{0} \rightarrow \operatorname{Vec}(k)$ is an equivalence of categories, in this case $\pi(\mathcal{T})$ can be identified with an affine group scheme in the usual sense.

Proposition 6.10. Let $\mathcal{M}$ be a Tannakian category over $\mathbb{Q}$ whose fundamental group $T$ is an algebraic group of multiplicative type, and let $\omega^{p}$ be a $\mathbb{Q}$-linear tensor functor $\mathcal{M} \rightarrow \operatorname{Mod}\left(\mathbb{A}_{f}^{p}\right)$ such that $\omega_{\ell}={ }_{\mathrm{df}} \omega^{p} \otimes_{\mathbb{A}_{f}^{p}} \mathbb{Q}_{\ell}$ is a $\mathbb{Q}_{\ell}$-valued fibre functor for all $\ell \neq p$. Then there exists a finite field extension $L$ of $\mathbb{Q}$, a fibre functor $\omega: \mathcal{M} \rightarrow \operatorname{Vec}(L)$, and an isomorphism $\omega \otimes_{L} \mathbb{A}_{f, L}^{p} \rightarrow \omega^{p} \otimes_{\mathbb{A}_{f}^{p}} \mathbb{A}_{f, L}^{p}$ of tensor functors $\mathcal{M} \rightarrow \operatorname{Mod}\left(\mathbb{A}_{f, L}^{p}\right)$

Proof. The hypotheses imply that $\omega^{p}(X)$ is a free $\mathbb{A}_{f}^{p}$-module for all $X$ (see the proof of 4.10 .

Assume first that $T$ is split over $\mathbb{Q}$ and that there exists a fibre functor $\omega: \mathcal{M} \rightarrow$ $\operatorname{Vec}(\mathbb{Q})$. We show in this case that there is an isomorphism $\omega \otimes_{\mathbb{Q}} \mathbb{A}_{f}^{p} \rightarrow \omega^{p}$. We use $\omega$ to identify $\mathcal{M}$ with $\operatorname{Rep}(T ; \mathbb{Q})$. Let $\Xi$ be a basis for the group $X^{*}(T)$ of characters of $T$, and let $X$ be the representation $X=\bigoplus_{\lambda \in \Xi} X_{\lambda}$ where $X_{\lambda}$ is a one-dimensional representation of $T$ with character $\lambda$. Choose a graded lattice $\Lambda$ in $X$, i.e., a $\mathbb{Z}$-lattice such that $\Lambda=\bigoplus_{\lambda \in \Xi} \Lambda \cap X_{\lambda}$. Likewise, choose a graded lattice $\Lambda_{f}$ in $\omega^{p}(X)$. Then $\Lambda_{\ell}={ }_{\mathrm{df}} \Lambda_{f} \otimes_{\mathbb{A}_{f}^{p}} \mathbb{Z}_{\ell}$ is a graded lattice in $\omega_{\ell}(X)$. As $T$ is a split torus, $H^{1}\left(\mathbb{Q}_{\ell}, T\right)=0$, and so the theory of Tannakian categories shows that there exists an isomorphism of fibre functors $\alpha_{\ell}: \omega \otimes_{\mathbb{Q}} \mathbb{Q}_{\ell} \rightarrow \omega_{\ell}$ which is uniquely determined up to an element of $T\left(\mathbb{Q}_{\ell}\right)$. We may choose $\alpha_{\ell}$ to map $\Lambda \otimes_{\mathbb{Z}} \mathbb{Z}_{\ell}$ onto $\Lambda_{\ell}$. Now the family $\left(\alpha_{\ell}\right)$ defines an isomorphism $\omega \otimes \mathbb{Q} \mathbb{A}_{f}^{p} \rightarrow \omega^{p}$.

In the general case, there will exist a finite field extension $L$ of $\mathbb{Q}$ such that $T$ splits over $L$ and such that there exists a fibre functor $\omega: \mathcal{M} \rightarrow \operatorname{Vec}(L)[8]$ 
6.20]. Then $\omega$ defines a fibre functor $\mathcal{M}_{(L)} \rightarrow \operatorname{Vec}(L)$ [10, 3.11], and the previous argument applied to $\mathcal{M}_{(L)}$ proves the general case of the proposition.

Example 6.11. Let $\mathcal{M}=\mathcal{M}_{\text {num }}(\mathbb{F} ; \mathbb{Q} ; \mathcal{S})$ be the Tannakian category over $\mathbb{Q}$ defined by some set $\mathcal{S}$ of varieties over $\mathbb{F}$ satisfying (4.1) and the condition $\left(^{*}\right)$ of $\S 5$, and assume that $\mathcal{M}$ has a $\otimes$-generator of the form $h V \oplus \mathbb{Q}(1)$ for some smooth projective variety $V$ over $\mathbb{F}$. Then the fundamental group of $\mathcal{M}$ is of multiplicative type if $V$ and its powers satisfy the Tate conjecture.

As $\mathcal{M}$ is semisimple, the fundamental group is reductive, and so it suffices to show that it is commutative. We choose a prime $\ell \neq p$ and show that $G={ }_{\text {df }} \omega_{\ell}(\pi(\mathcal{M})) \cong$ $\underline{\text { Aut }}^{\otimes}\left(\omega_{\ell}\right)$ is commutative. Because of our assumption on $V$, the algebraic cohomology classes on the powers of $V$ are the Tate classes, and so $G$ is the group of automorphisms of the $\mathbb{Q}_{\ell}$-vector space $\omega_{\ell}(h V \oplus \mathbb{Q}(1))=\left(\bigoplus_{r} H^{r}\left(V, \mathbb{Q}_{\ell}\right)\right) \oplus \mathbb{Q}_{\ell}(1)$ fixing the Tate classes on all powers of $V$. Let $\pi$ be the Frobenius endomorphism of $\left(\bigoplus_{r} H^{r}\left(V, \mathbb{Q}_{\ell}\right)\right) \oplus \mathbb{Q}_{\ell}(1)$ defined by some model of $V$ over a finite subfield of $\mathbb{F}$. Then the Tate classes on the powers of $V$ are (by definition) the cohomology classes in $H^{2 r}\left(V^{m}, \mathbb{Q}_{\ell}(r)\right), m, r \in \mathbb{N}$, fixed by some power of $\pi$. Let $G_{n}$ be the Zariski closure of the subgroup generated by $\pi^{n}$. Then $G_{n} \supset G_{n^{\prime}}$ if $n \mid n^{\prime}$, and the groups $G_{n}$ become constant for $n$ sufficiently divisible. According to [10 3.1c], $G$ equals $G_{n}$ for $n$ sufficiently divisible. In particular, it is the Zariski closure of a commutative subgroup of $\mathrm{GL}\left(\omega_{\ell}(h V \oplus \mathbb{Q}(1))\right)$, which implies that it is commutative.

The category $\mathcal{M}\left(\mathbb{F}_{q} ; \mathbb{Z}\right)$. Let $\mathcal{S}$ be a class of smooth projective varieties over $\mathbb{F}_{q}$ satisfying (4.1) and the condition $(*)$ in $\S 5$, and let $\mathcal{M}\left(\mathbb{F}_{q} ; \mathbb{Z}\right)$ be the corresponding category of motives (5.1). Recall (5.15) that $\mathcal{M}\left(\mathbb{F}_{q} ; \mathbb{Z}\right)$ has internal Homs.

Lemma 6.12. (a) An object in $\mathcal{M}\left(\mathbb{F}_{q} ; \mathbb{Z}\right)$ is reflexive if and only if it is torsion-free.

(b) Every object $X$ in $\mathcal{M}\left(\mathbb{F}_{q} ; \mathbb{Z}\right)$ is a quotient of a reflexive object.

Proof. (a) This is obvious from (1.5 $\mathrm{k})$ and $(1.8 \mathrm{a})$.

(b) When $X$ is torsion with trivial $\Gamma$-action, the proofs of $(1.5 \mathrm{~b})$ and $(1.8 \mathrm{~b})$ show that $X$ is a quotient of direct sum of Lefschetz motives $\mathbb{L}^{\otimes n}$. Since $X$ will decompose into the direct sum of such a torsion motive and a torsion-free motive over a finite extension $\mathbb{F}_{q^{\prime}}$ of $\mathbb{F}_{q}$ (see 1.4 and 1.7), to complete the proof it remains to define a suitable "norm" map $\Pi: \mathcal{M}\left(\mathbb{F}_{q^{\prime}} ; \mathbb{Z}\right) \rightarrow \mathcal{M}\left(\mathbb{F}_{q} ; \mathbb{Z}\right)$ (cf. the proof of $1.5 \mathrm{~b}$ ), and for this it suffices to define compatible $\Pi$ 's for $\mathcal{M}\left(\mathbb{F}_{q^{\prime}} ; \mathbb{Q}\right)$ and for the realization categories. For $\mathcal{R}\left(\mathbb{F}_{q^{\prime}} ; \mathbb{Z}_{\ell}\right)(\ell \neq p)$, we take $\Pi$ to be $\operatorname{Ind}_{\mathrm{Gal}\left(\mathbb{F} / \mathbb{F}_{q}\right)}^{\left.\mathrm{Gal} / \mathbb{F}^{\prime}\right)}$. For an $F$-crystal $\Lambda$ over $\mathbb{F}_{q^{\prime}}$, descent theory shows that $\bigoplus_{\tau \in \operatorname{Gal}\left(\mathbb{F}_{q^{\prime}} / \mathbb{F}_{q}\right)} \tau \Lambda$ arises from an $F$-crystal over $\mathbb{F}_{q}$. This leads to a functor $\Pi: \mathcal{R}\left(\mathbb{F}_{q^{\prime}} ; \mathbb{Z}_{p}\right) \rightarrow \mathcal{R}\left(\mathbb{F}_{q} ; \mathbb{Z}_{p}\right)$. For a $V \in \mathcal{S}\left(\mathbb{F}_{q^{\prime}}\right)$, the composite $V \rightarrow \operatorname{Spec} \mathbb{F}_{q^{\prime}} \rightarrow \operatorname{Spec} \mathbb{F}_{q}$ lies in $\mathcal{S}\left(\mathbb{F}_{q}\right)$ (by the compatibility condition). This leads to a functor $\Pi: \mathcal{C} \mathcal{V}_{\text {num }}^{0}\left(\mathbb{F}_{q^{\prime}}\right) \rightarrow \mathcal{C} \mathcal{V}_{\text {num }}^{0}\left(\mathbb{F}_{q}\right)$, which extends to a functor $\Pi: \mathcal{M}\left(\mathbb{F}_{q^{\prime}} ; \mathbb{Q}\right) \rightarrow \mathcal{M}\left(\mathbb{F}_{q} ; \mathbb{Q}\right)$. That these functors are compatible follows from the basic properties of étale and crystalline cohomology.

Theorem 6.13. The category $\mathcal{M}\left(\mathbb{F}_{q} ; \mathbb{Z}\right)$ is Tannakian provided the varieties in $\mathcal{S}$ satisfy the Tate conjecture.

Proof. We replace $\mathcal{S}\left(\mathbb{F}_{q}\right)$ with a subset $\mathcal{S}$ generated by a finite set of varieties and prove that the resulting category $\mathcal{M}=\mathcal{M}\left(\mathbb{F}_{q} ; \mathbb{Z} ; \mathcal{S}\right)$ is an algebraic Tannakian category. This will follow from Proposition 6.7 once we have shown that $\mathcal{M}_{(R)}$ 
admits an $R$-valued fibre functor for some Dedekind domain $R$ finite and flat over $\mathbb{Z}$.

Because of (6.11), we can apply Proposition 6.10 to $\mathcal{M}(\mathbb{F} ; \mathbb{Z} ; \mathcal{S})$, which shows that there exists a finite field extension $L$ of $\mathbb{Q}$, a fibre functor $\omega_{0}: \mathcal{M}(\mathbb{F} ; \mathbb{Z} ; \mathcal{S}) \rightarrow$ $\operatorname{Vec}(L)$, and an isomorphism $\xi^{p}: L \otimes_{\mathbb{Q}} \omega^{p} \rightarrow \mathbb{A}_{f}^{p} \otimes_{\mathbb{Q}} \omega_{0}$. When composed with $\mathcal{M}\left(\mathbb{F}_{q} ; \mathbb{Z} ; \mathcal{S}\right) \rightarrow \mathcal{M}(\mathbb{F} ; \mathbb{Z} ; \mathcal{S}), \omega_{0}$ becomes a fibre functor on $\mathcal{M} ;$ moreover, $\xi$ becomes an isomorphism of functors on $\mathcal{M}$.

Let $\omega_{p}$ be the functor $X \mapsto \mathbb{Q}_{p} \otimes_{\mathbb{Z}_{p}} X_{p}$ (regarded as a $B\left(\mathbb{F}_{q}\right)$-module). Then $\omega_{p}$ and $\mathbb{Q}_{p} \otimes_{\mathbb{Q}} \omega_{0}$ are both fibre functors on $\mathcal{M}$, and so become isomorphic over a finite extension of $B\left(\mathbb{F}_{q}\right)[8,1.10,6.20]$. Therefore, after possibly extending $L$, we may suppose that there exists an isomorphism $\xi_{p}: L \otimes_{\mathbb{Q}} \omega_{p} \rightarrow \mathbb{Q}_{p} \otimes_{\mathbb{Q}} \omega_{0}$. Let $R$ be the integral closure of $\mathbb{Z}$ in $L$, and let $\hat{R}$ be its profinite completion.

For $X_{0}$ in $\mathcal{M}_{(L)}$, there are two actions of $L$ on $\omega_{0}\left(X_{0}\right)$, namely, that coming from the action of $L$ on $\omega_{0}$ and that coming from the action of $L$ on $X_{0}$. Define

$$
\omega_{1}\left(X_{0}\right)=L \otimes_{L \otimes_{\mathbb{Q}} L} \omega_{0}\left(X_{0}\right) .
$$

Then $\omega_{1}$ is an $L$-valued fibre functor on $\mathcal{M}_{(L)}$ [10, 3.11].

For $\left(X, i_{X}\right)$ in $\mathcal{M}_{(R)}$, set

$$
\omega_{2}(X)=\left(X^{p}, \omega^{\prime}\left(X_{p}\right)\right)
$$

where $\omega^{\prime}$ is the functor defined in $(6.9 \mathrm{l})$. Then $\omega_{2}$ is an $\hat{R}$-valued fibre functor on $\mathcal{M}_{(R)}$, and $\xi$ defines an isomorphism $L \otimes_{\mathbb{Q}} \omega_{2} \rightarrow \mathbb{A}_{f} \otimes_{\mathbb{Q}} \omega_{1}$.

Thus, we have defined an exact tensor functor

$$
X \mapsto\left(\omega_{2}(X), \omega_{1}\left(X_{0}\right), \xi\right): \mathcal{M}_{(R)} \rightarrow \operatorname{Modf}(\hat{R}) \times_{\operatorname{Modf}\left(\mathbb{A}_{f} \otimes R\right)} \operatorname{Modf}(L) .
$$

Its image lies in the full subcategory of triples whose $\operatorname{Modf}(\hat{R})$-component has finite torsion, which can be identified with the category $\operatorname{Modf}(R)$. It is therefore an $R$-valued fibre functor on $\mathcal{M}\left(\mathbb{F}_{q} ; \mathbb{Z}\right)_{(R)}$.

Remark 6.14. (a) If $\mathcal{M}_{\text {num }}(\mathbb{F} ; \mathbb{Q} ; \mathcal{S})$ has tensor generator of the form $h A \oplus \mathbb{Q}(1)$ with $A$ an abelian variety, then its fundamental group is of multiplicative type because it is contained in the Lefschetz group of $A$ (see [41, especially $\S 7]$ ). Therefore, $\mathcal{M}\left(\mathbb{F}_{q} ; \mathbb{Z} ; \mathcal{S}\right)$ is Tannakian if the connected varieties in $\mathcal{S}$ are products of abelian varieties, projective varieties, and varieties of dimension zero (whether or not they satisfy the Tate conjecture).

(b) We expect that Proposition 6.10 holds more generally for Tannakian categories whose fundamental groups are reductive (not necessarily connected). If so, Theorem 6.13 holds without the Tate conjecture condition, and, moreover, $\mathcal{M}\left(k ; \mathbb{Z}\left[\frac{1}{p}\right] ; \mathcal{S}\right)$ will always be Tannakian.

Remark 6.15. If $\mathcal{M}(k ; \mathbb{Q})$ is as in 5.25 or 5.27 and $k$ is finite, or as in 5.26, then $\mathcal{M}(k ; \mathbb{Z})$ is Tannakian. In the first two cases, this can be proved as in Theorem 6.13, and in the third case, there is a Betti fibre functor on $\mathcal{M}(k ; \mathbb{Z})$.

Stacks of categories of motives. We refer the reader to [47, I, 4.5] for the notion of tensor fibred category $p: \mathcal{M} \rightarrow \mathcal{E}$. Briefly, it is a fibred category $p: \mathcal{M} \rightarrow \mathcal{E}$ endowed with an $\mathcal{E}$-bifunctor $\otimes: \mathcal{M} \times \mathcal{E} \mathcal{M} \rightarrow \mathcal{M}$ and compatible associativity and commutativity constraints for which there exists a cartesian section to $p$ giving identity objects in each fibre; thus, for each $E$ in $\mathcal{E}$, the fibre $\mathcal{M}_{E}$ is a tensor category. When $\mathcal{E}$ is endowed with a topology (in the sense of Grothendieck) and 
$p: \mathcal{M} \rightarrow \mathcal{E}$ is a stack, we call it a stack of tensor categories. When the fibres are abelian categories and the inverse image functors are exact, we call $p: \mathcal{M} \rightarrow \mathcal{E}$ a stack of abelian tensor categories. Finally, when $\operatorname{End}(\mathbb{1})=R$ for each identity object $\mathbb{1}$ in each fibre, we call $p: \mathcal{M} \rightarrow \mathcal{E}$ a stack of abelian tensor $R$-categories. In particular, then each fibre is an abelian tensor $R$-category.

For a scheme $S=\coprod$ Spec $k_{i}$, finite and étale over $\mathbb{F}_{q}$, let $\mathcal{M}(S ; \mathbb{Z})=\prod \mathcal{M}\left(k_{i} ; \mathbb{Z}\right)$.

Theorem 6.16. Let $\mathbf{E t}_{\mathbb{F}_{q}}$ be the category of all schemes finite and étale over $\mathbb{F}_{q}$, and endow $\mathbf{E t}_{\mathbb{F}_{q}}$ with the étale topology. For $k=\mathbb{F}_{q}$ or $\mathbb{F}$, let $\mathcal{M}(k ; \mathbb{Q})$ be as in (5.20) (in particular, we assume the Tate conjecture for $\mathcal{M}(\mathbb{F} ; \mathbb{Q})$ ). The categories $\mathcal{M}(-; \mathbb{Z})$ form (in a natural way) the fibres of a stack of abelian tensor $\mathbb{Z}$-categories over $\mathbf{E t}_{\mathbb{F}_{q}}$.

Proof. Immediate consequence of Proposition 5.20,

\section{Some SPECTRAL SEQUEnCES}

Abstract spectral sequences. We prove an abstract version of Tate's spectral sequence [39, I, 0.3]. Fix a field $k$, and let $\mathbf{E t}_{k}$ be the category of all schemes finite and étale over $k$. Also, fix a noetherian $\operatorname{ring} R$, and let $\mathcal{M} \rightarrow \mathbf{E t}_{k}$ be a stack of noetherian abelian tensor $R$-categories over $\mathbf{E t}_{k}$.

7.1. When $X$ and $Y$ are objects of an abelian category $\mathcal{A}, \operatorname{Ext}^{r}(X, Y)$ denotes the Yoneda extension group [43, VII]. It agrees with the group defined in terms of the derived category [56, III, 3.2.12]. When $\mathcal{A}$ is noetherian, the Yoneda extension group also agrees with that defined using injective resolutions in the ind-category $\operatorname{Ind}(\mathcal{A})([44$, p. 235]; see also [24]).

7.2. An object of a tensor category is trivial if it is a quotient of a finite direct sum of copies of $\mathbb{1}$, and an object $Y$ of an abelian tensor category is flat if the functor $X \mapsto X \otimes Y$ is exact. When $X$ and $Y$ are objects of an abelian tensor category over $R$, we write

$$
\operatorname{Tor}_{i}^{R}(X, Y)=0 \text { for all } i>0
$$

to mean that $Y$ has a resolution

$$
\cdots \rightarrow Y^{1} \rightarrow Y^{0} \rightarrow Y \rightarrow 0
$$

by flat objects which remains exact when it has been tensored by $X$.

7.3. To avoid inessential difficulties, we assume that $\mathcal{M} / \mathbf{E t}_{k}$ is a split fibred category, i.e., that it is equipped with compatible inverse image functors [19, I, 1.0.3]. According to a theorem of Giraud [18, §5] every fibred category is equivalent with a split fibred category. Let $k^{\prime}$ be a finite Galois extension of $k$ with Galois group $\Gamma$, and let $X \mapsto X^{\prime}: \mathcal{M}(k) \rightarrow \mathcal{M}\left(k^{\prime}\right)$ be the inverse image functor. Because $\mathcal{M} / \mathbf{E t}_{k}$ is split, to give a $k^{\prime} / k$ descent datum on an object of $\mathcal{M}\left(k^{\prime}\right)$ is to give an action of $\Gamma$. Therefore, $X \mapsto X^{\prime}$ defines an equivalence of $\mathcal{M}(k)$ with the category $\mathcal{M}\left(k^{\prime} ; \Gamma\right)$ of objects of $\mathcal{M}\left(k^{\prime}\right)$ equipped with an action of $\Gamma$. Explicitly, for objects $X, Y$ of $\mathcal{M}(k)$, the actions of $\Gamma$ on $X^{\prime}$ and $Y^{\prime}$ define an action of $\Gamma$ on $\operatorname{Hom}_{\mathcal{M}\left(k^{\prime}\right)}\left(X^{\prime}, Y^{\prime}\right)$ and

$$
\operatorname{Hom}_{\mathcal{M}(k)}(X, Y) \cong \operatorname{Hom}_{\mathcal{M}\left(k^{\prime}\right)}\left(X^{\prime}, Y^{\prime}\right)^{\Gamma} .
$$

The functor $\operatorname{Hom}_{\mathcal{M}\left(k^{\prime}\right)}(\mathbb{1},-)$ is an equivalence from the full subcategory of $\mathcal{M}^{0}\left(k^{\prime}\right)$ of trivial objects in $\mathcal{M}\left(k^{\prime}\right)$ to $\operatorname{Modf}(R)$, and hence from $\mathcal{M}^{0}\left(k^{\prime} ; \Gamma\right)$ to $\operatorname{Modf}(R[\Gamma])$. Let $N \mapsto \underline{N}: \operatorname{Modf}(R[\Gamma]) \rightarrow \mathcal{M}^{0}\left(k^{\prime} ; \Gamma\right)$ denote a quasi-inverse. Then $\underline{N} \cong N \otimes_{R}$ 
$\mathbb{1}$ (external tensor product (6.1) in $\mathcal{M}^{0}\left(k^{\prime}\right)$, with $\Gamma$ acting through $N$ ) because $\operatorname{Hom}_{\mathcal{M}\left(k^{\prime}\right)}\left(\mathbb{1}, N \otimes_{R} \mathbb{1}\right) \cong N$ as $R[\Gamma]$-modules. The functor

$$
X \mapsto N(X) \stackrel{\mathrm{df}}{=} \operatorname{Hom}\left(\mathbb{1}, X^{\prime}\right)
$$

is an equivalence from the full subcategory $\mathcal{M}^{1}(k)$ of $\mathcal{M}(k)$ of objects becoming trivial in $\mathcal{M}\left(k^{\prime}\right)$ to $\operatorname{Modf}(R[\Gamma])$.

Proposition 7.4. Let $k^{\prime}$ be a finite Galois extension of $k$ with Galois group $\Gamma$. With the above notations, for all $X, Y, Z$ in $\mathcal{M}(k)$ such that $X^{\prime}$ is trivial and $\operatorname{Tor}_{i}^{R}(X, Y)=0$ for all $i>0$, there is a spectral sequence

$$
\operatorname{Ext}_{R[\Gamma]}^{r}\left(N(X), \operatorname{Ext}_{\mathcal{M}\left(k^{\prime}\right)}^{s}\left(Y^{\prime}, Z^{\prime}\right)\right) \Longrightarrow \operatorname{Ext}_{\mathcal{M}(k)}^{r+s}(X \otimes Y, Z) .
$$

After a few preliminaries, we obtain this as the spectral sequence of a composite of functors.

Lemma 7.5. 11 For any $X, Y, Z \in \mathrm{ob} \mathcal{M}(k)$ with $X^{\prime}$ trivial, there is a canonical isomorphism

$$
\operatorname{Hom}_{R[\Gamma]}\left(N(X), \operatorname{Hom}_{\mathcal{M}\left(k^{\prime}\right)}\left(Y^{\prime}, Z^{\prime}\right)\right) \cong \operatorname{Hom}_{\mathcal{M}(k)}(X \otimes Y, Z) .
$$

Proof. For any finitely generated $R$-module $N$, we have an isomorphism (see 6.1)

$$
\operatorname{Hom}_{R}\left(N, \operatorname{Hom}_{\mathcal{M}\left(k^{\prime}\right)}\left(Y^{\prime}, Z^{\prime}\right)\right) \cong \operatorname{Hom}_{\mathcal{M}\left(k^{\prime}\right)}\left(N \otimes_{R} Y^{\prime}, Z^{\prime}\right) \text {. }
$$

From the definition of the external tensor product $\otimes_{R}$, it is clear that

$$
\left(N \otimes_{R} T_{1}\right) \otimes T_{2} \cong N \otimes_{R}\left(T_{1} \otimes T_{2}\right)
$$

for objects $T_{1}, T_{2}$ of $\mathcal{M}\left(k^{\prime}\right)$. Therefore,

$$
N \otimes_{R} Y^{\prime} \cong N \otimes_{R}\left(\mathbb{1} \otimes Y^{\prime}\right) \cong\left(N \otimes_{R} \mathbb{1}\right) \otimes Y^{\prime} .
$$

Let $N=N(X)$. Then $N \otimes_{R} \mathbb{1} \cong \underline{N} \cong X^{\prime}$, and so (11) becomes

$$
\operatorname{Hom}_{R}\left(N(X), \operatorname{Hom}_{\mathcal{M}\left(k^{\prime}\right)}\left(Y^{\prime}, Z^{\prime}\right)\right) \cong \operatorname{Hom}_{\mathcal{M}\left(k^{\prime}\right)}\left(X^{\prime} \otimes_{R} Y^{\prime}, Z^{\prime}\right) .
$$

On taking $\Gamma$-invariants, we obtain (10).

In the remainder of the proof, we abbreviate $\operatorname{Ext}_{\mathcal{M}(k)}$ and $\operatorname{Ext}_{\operatorname{Ind} \mathcal{M}(k)}$ to $\operatorname{Ext}_{k}$.

Lemma 7.6. If $I$ is an injective object of $\operatorname{Ind}(\mathcal{M}(k))$ and $Y$ is a flat object of $\mathcal{M}(k)$, then $\operatorname{Hom}_{k^{\prime}}(Y, I)$ is an injective $R[\Gamma]$-module.

Proof. Because $R[\Gamma]$ is left noetherian, it suffices to show that the functor $\operatorname{Hom}_{R[\Gamma]}\left(-, \operatorname{Hom}_{k^{\prime}}(Y, I)\right)$ is exact on finitely generated $R[\Gamma]$-modules. In view of (7.3), we can regard this as a functor on $\mathcal{M}^{1}(k)$, and then Lemma 7.5 expresses it as the composite of two exact functors.

Lemma 7.7. If I is injective in $\operatorname{Ind}(\mathcal{M}(k))$, then $I^{\prime}$ is injective in $\operatorname{Ind}\left(\mathcal{M}\left(k^{\prime}\right)\right)$.

Proof. For an object $X$ in $\mathcal{M}\left(k^{\prime}\right), R[\Gamma] \otimes_{R} X$ (external tensor product in $\mathcal{M}\left(k^{\prime}\right)$ ) becomes an object of $\mathcal{M}\left(k^{\prime} ; \Gamma\right)$ through the action of $\Gamma$ on $R[\Gamma]$. As $\mathcal{M}(k ; \Gamma) \simeq$ $\mathcal{M}(k), R[\Gamma] \otimes_{R} X$ can be regarded as an object of $\mathcal{M}(k)$. This functor $X \mapsto$ $R[\Gamma] \otimes_{R} X: \mathcal{M}\left(k^{\prime}\right) \rightarrow \mathcal{M}(k)$ is an exact left adjoint to $Y \mapsto Y^{\prime}: \mathcal{M}(k) \rightarrow \mathcal{M}\left(k^{\prime}\right)$.

Lemma 7.8. Let $X, Y$ be as in (7.4), and let $I$ be an injective object of $\operatorname{Ind}(\mathcal{M}(k))$. Then $\operatorname{Ext}_{R[\Gamma]}^{r}\left(N(X), \operatorname{Hom}_{k^{\prime}}\left(Y^{\prime}, I^{\prime}\right)\right)=0$ for $r>0$.

\footnotetext{
${ }^{11}$ We thank the referee for pointing out to us that this lemma (hence the proposition) does not require that the categories have internal Homs.
} 
Proof. Let $Y^{\bullet} \rightarrow Y$ be a flat resolution of $Y$ that remains a resolution when tensored with $X$, and let $I$ be an injective object of $\operatorname{Ind}(\mathcal{M}(k))$. Then $I^{\prime}$ is injective (see [7.7), and so

$$
\operatorname{Hom}_{k^{\prime}}\left(Y^{\prime}, I^{\prime}\right) \rightarrow \operatorname{Hom}_{k^{\prime}}\left(Y^{\bullet \prime}, I^{\prime}\right)
$$

is a resolution of $\operatorname{Hom}_{k^{\prime}}\left(Y^{\prime}, I^{\prime}\right)$. In fact (see 7.6), it is an injective resolution of $\operatorname{Hom}_{k^{\prime}}\left(Y^{\prime}, I^{\prime}\right)$, which we shall use to compute $\operatorname{Ext}_{R[\Gamma]}^{r}\left(N(X), \operatorname{Hom}_{k^{\prime}}\left(Y^{\prime}, I^{\prime}\right)\right)$. Lemma [7.5] shows that $\operatorname{Hom}_{R[\Gamma]}\left(N(X), \operatorname{Hom}_{k^{\prime}}\left(Y^{\bullet \prime}, I^{\prime}\right)\right) \cong \operatorname{Hom}_{k}\left(X \otimes Y^{\bullet}, I\right)$, which is exact except at the zeroth position. Therefore, $\operatorname{Ext}_{R[\Gamma]}^{r}\left(N(X), \operatorname{Hom}_{k^{\prime}}\left(Y^{\prime}, I^{\prime}\right)\right)=0$ for $r>0$.

We now prove the proposition. Lemma 7.5 shows that $\operatorname{Hom}_{k}(X \otimes Y,-)$ is the composite of the functors $\operatorname{Hom}_{k^{\prime}}\left(Y^{\prime},-\right)$ and $\operatorname{Hom}_{R[\Gamma]}(N(X),-)$, and Lemma 7.8 shows that the first of these sends injective objects to objects that are acyclic for the second functor. Therefore, we obtain the spectral sequence as that attached to the composite of functors.

Corollary 7.9. Let $\bar{k}$ be a Galois extension of $k$ (possibly infinite), and let $\mathcal{M}(\bar{k})=$ $\lim _{k^{\prime}} \mathcal{M}\left(k^{\prime}\right)$ where $k^{\prime}$ runs over the finite Galois extensions of $k$ in $\bar{k}$. Denote the inverse image functor $\mathcal{M}(k) \rightarrow \mathcal{M}(\bar{k})$ by $X \mapsto \bar{X}$, and let $\Gamma=\operatorname{Gal}(\bar{k} / k)$. Let $Y$ be an object of $\mathcal{M}(k)$ that admits a resolution by flat objects. For all $Z$ in $\mathcal{M}(k)$, there is a spectral sequence

$$
H^{r}\left(\Gamma, \operatorname{Ext}_{\mathcal{M}(\bar{k})}^{s}(\bar{Y}, \bar{Z})\right) \Longrightarrow \operatorname{Ext}_{\mathcal{M}(k)}^{r+s}(Y, Z) .
$$

Proof. The condition on $Y$ implies that $\operatorname{Tor}_{i}^{R}(\mathbb{1}, Y)=0$ for all $i>0$. When $\bar{k}$ is finite over $k$, the two definitions of $\mathcal{M}(\bar{k})$ coincide, and we obtain the spectral sequence by taking $X=\mathbb{1}$ in Proposition 7.4. When $\bar{k}$ is of infinite degree over $k$, we pass to the limit over subfields $k^{\prime} \subset \bar{k}$ that are finite and Galois over $k$. It is clear that $\operatorname{Ext}_{\mathcal{M}(\bar{k})}^{s}(\bar{Y}, \bar{Z}) \cong \underline{\lim }_{k^{\prime}} \operatorname{Ext}_{\mathcal{M}\left(k^{\prime}\right)}^{s}\left(Y^{\prime}, Z^{\prime}\right)$, for example, for the Yoneda Exts, because our direct limits of categories are the naive ones (1.2) and the functor $\mathcal{M}\left(k^{\prime}\right) \rightarrow \mathcal{M}\left(k^{\prime \prime}\right)$ is exact for finite extensions $k^{\prime \prime} / k^{\prime}$.

Spectral sequences at $\ell$. The categories $\mathcal{R}\left(\mathbb{F}_{q} ; \mathbb{Z}_{\ell}\right)$ for varying $q$ form a stack of

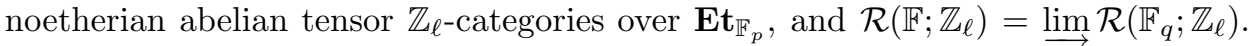
Moreover, every object of $\mathcal{R}\left(\mathbb{F}_{q} ; \mathbb{Z}_{\ell}\right)$ admits a (short) resolution by flat objects (1.5). Thus, Corollary 7.9 has the following special case.

Proposition 7.10. Let $\Gamma=\operatorname{Gal}\left(\mathbb{F} / \mathbb{F}_{q}\right)$, and let $M \mapsto \bar{M}$ denote the functor $\mathcal{R}\left(\mathbb{F}_{q} ; \mathbb{Z}_{\ell}\right) \rightarrow \mathcal{R}\left(\mathbb{F} ; \mathbb{Z}_{\ell}\right)$. For $M, N$ in $\mathcal{R}\left(\mathbb{F}_{q} ; \mathbb{Z}_{\ell}\right)$, there is a spectral sequence

$$
H^{r}\left(\Gamma, \operatorname{Ext}_{\mathcal{R}\left(\mathbb{F} ; \mathbb{Z}_{\ell}\right)}^{s}(\bar{M}, \bar{N})\right) \Longrightarrow \operatorname{Ext}_{\mathcal{R}\left(\mathbb{F}_{q} ; \mathbb{Z}_{\ell}\right)}^{r+s}(M, N) .
$$

Denote Exts in $\operatorname{Mod}\left(\mathbb{Z}_{\ell}\right)$ by $\operatorname{Ext}_{\mathbb{Z}_{\ell}}$.

Proposition 7.11. Let $\Gamma=\operatorname{Gal}\left(\mathbb{F} / \mathbb{F}_{q}\right)$. For $M, N$ in $\mathcal{R}\left(\mathbb{F}_{q} ; \mathbb{Z}_{\ell}\right)$, there is a spectral sequence

$$
H_{\text {cts }}^{r}\left(\Gamma, \operatorname{Ext}_{\mathbb{Z}_{\ell}}^{s}(M, N)\right) \Longrightarrow \operatorname{Ext}_{\mathcal{R}\left(\mathbb{F}_{q} ; \mathbb{Z}_{\ell}\right)}^{r+s}(M, N) .
$$

Here $H_{\mathrm{cts}}^{r}$ is computed using continuous cochains relative to the $\ell$-adic topology on $\operatorname{Ext}_{\mathbb{Z}_{\ell}}^{s}(M, N)$. 
Proof. Let $\mathcal{R}=\mathcal{R}\left(\mathbb{F}_{q} ; \mathbb{Z}_{\ell}\right)$. When $N$ is finite, (13) will become (12) once we have shown that the natural map

$$
\left.\operatorname{Ext}_{\mathcal{R}}^{j}(M, N)\right) \rightarrow \operatorname{Ext}_{\mathbb{Z}_{\ell}}^{j}(M, N)
$$

is an isomorphism. When $M$ is finite, this is obvious (with no condition on $N$ ), because it is true for $j=0$ and the forgetful functor Ind $\mathcal{R} \rightarrow \operatorname{Mod}\left(\mathbb{Z}_{\ell}\right)$ is exact and preserves injectives ( $(1.5 \mathrm{~b})$ implies that every injective in Ind $\mathcal{R}$ is $\ell$-divisible). The $\operatorname{Ext}(M,-)$ sequences of

$$
\begin{aligned}
& 0 \rightarrow M \stackrel{\ell^{n}}{\rightarrow} M \rightarrow M / \ell^{n} M \rightarrow 0, \quad \ell^{n} N=0, \\
& 0 \rightarrow M_{\text {tors }} \rightarrow M \rightarrow M / M_{\text {tors }} \rightarrow 0
\end{aligned}
$$

allow us to deduce it, first for all torsion-free $M$, and then for all $M$.

For $M$ in $\mathcal{R}$ and a projective system $\left(N_{n}\right)_{n \in \mathbb{N}}$ in $\mathcal{R}^{\mathbb{N}}$, there is a canonical isomorphism

$$
\operatorname{Hom}\left(M, \varliminf_{\varliminf} N_{n}\right) \cong \lim _{\longleftarrow} \operatorname{Hom}\left(M, N_{n}\right) .
$$

Let $F\left(\left(N_{n}\right)_{n}\right)$ be the common value of the two sides. Then $F$ is a functor $\mathcal{R}^{\mathbb{N}} \rightarrow \mathbf{A b}$ which (15) expresses as a composite in two ways, and it can be shown that this leads to two spectral sequences

$$
\begin{aligned}
E_{2}^{i j} & =\operatorname{Ext}_{\mathcal{R}}^{i}\left(M, \lim ^{j} N_{n}\right) \Longrightarrow R^{i+j} F\left(\left(N_{n}\right)_{n}\right), \\
E_{2}^{i, j} & =\lim ^{i} \operatorname{Ext}_{\mathcal{R}}^{j}\left(M, N_{n}\right) \Longrightarrow R^{i+j} F\left(\left(N_{n}\right)_{n}\right)
\end{aligned}
$$

(cf. [46]; 27, 4.3]).

When we apply this to the inverse system $\left(N^{\left(\ell^{n}\right)}\right)_{n}$, the higher inverse limits in the $E_{2}$-terms vanish because the groups $\operatorname{Ext}_{\mathcal{R}}^{j}\left(M, N^{\left(\ell^{n}\right)}\right)$ and $N^{\left(\ell^{n}\right)}$ are finite. Moreover, $\lim _{\longleftarrow} N^{\left(\ell^{n}\right)} \cong N$, and so this shows that

$$
\operatorname{Ext}_{\mathcal{R}}^{i}(M, N) \cong \underset{n}{\lim _{n}} \operatorname{Ext}_{\mathcal{R}}^{i}\left(M, N^{\left(\ell^{n}\right)}\right)
$$

Similarly,

and so [54, 2.2]

$$
\operatorname{Ext}_{\mathbb{Z}_{\ell}}^{j}(M, N) \cong{\underset{n}{n}}_{\lim _{n}} \operatorname{Ext}_{\mathbb{Z}_{\ell}}^{j}\left(M, N^{\left(\ell^{n}\right)}\right)
$$

$$
H_{\mathrm{cts}}^{i}\left(\Gamma, \operatorname{Ext}_{\mathbb{Z}_{\ell}}^{j}(M, N)\right) \cong{\underset{n}{n}}_{\lim } H_{\mathrm{cts}}^{i}\left(\Gamma, \operatorname{Ext}_{\mathbb{Z}_{\ell}}^{j}\left(M, N^{\left(\ell^{n}\right)}\right)\right) .
$$

Therefore, (13) can be obtained by passing to the limit in the spectral sequences

$$
H_{\text {cts }}^{r}\left(\Gamma, \operatorname{Ext}_{\mathbb{Z}_{\ell}}^{s}\left(M, N^{\left(\ell^{n}\right)}\right)\right) \Longrightarrow \operatorname{Ext}_{\mathcal{R}}^{r+s}\left(M, N^{\left(\ell^{n}\right)}\right) .
$$

Spectral sequences at $p$.

Lemma 7.12. For torsion-free objects $M, N$ in $\mathcal{R}^{+}\left(\mathbb{F} ; \mathbb{Z}_{p}\right)$, $\operatorname{Ext}_{\mathcal{R}^{+}\left(\mathbb{F} ; \mathbb{Z}_{p}\right)}^{2}(M, N)$ is torsion-free.

Proof. From the exact sequences

$$
0 \rightarrow \operatorname{Ext}_{\mathcal{R}^{+}}^{1}(M, N)^{\left(p^{n}\right)} \rightarrow \operatorname{Ext}_{\mathcal{R}^{+}}^{1}\left(M, N^{\left(p^{n}\right)}\right) \rightarrow \operatorname{Ext}_{\mathcal{R}^{+}}^{2}(M, N)_{p^{n}} \rightarrow 0
$$

we obtain a surjection

$$
\underset{n}{\lim _{\longrightarrow}} \operatorname{Ext}_{\mathcal{R}^{+}}^{1}\left(M, N^{\left(p^{n}\right)}\right) \rightarrow \operatorname{Ext}_{\mathcal{R}^{+}}^{2}(M, N)_{\text {tors }}
$$


Let $\mathcal{R}_{p^{n}}^{+}$denote the full subcategory of $\mathcal{R}^{+}\left(\mathbb{F} ; \mathbb{Z}_{p}\right)$ of objects killed by $p^{n}$. An argument using the analogue of the diagram (23) shows that the map

$$
s: \operatorname{Ext}_{\mathcal{R}^{+}}^{1}\left(M, N^{\left(p^{n}\right)}\right) \rightarrow \operatorname{Ext}_{\mathcal{R}_{p^{n}}^{+}}^{1}\left(M^{\left(p^{n}\right)}, N^{\left(p^{n}\right)}\right)
$$

that replaces each term $E$ in a short exact sequence with $E^{\left(p^{n}\right)}$ is an isomorphism. Therefore, it remains to show that

$$
\underset{n}{\lim } \operatorname{Ext}_{\mathcal{R}_{p^{n}}^{+}}^{1}\left(M^{\left(p^{n}\right)}, N^{\left(p^{n}\right)}\right)=0 .
$$

We claim (i) that the map

$$
\operatorname{Ext}_{\mathbf{C r y s}^{+}(\mathbb{F})}^{1}\left(M_{W(\mathbb{F})}, N_{W(\mathbb{F})}\right) \rightarrow \operatorname{Ext}_{\mathcal{R}_{p^{n}}^{+}}^{1}\left(M^{\left(p^{n}\right)}, N^{\left(p^{n}\right)}\right)
$$

that replaces each term $E$ in a short exact sequence by $E^{\left(p^{n}\right)}$ is surjective, and (ii) that $\operatorname{Ext}_{\text {Crys }^{+}(\mathbb{F})}^{1}(M, N)$ is a torsion group. Together, (i) and (ii) imply (17), because (i) gives a surjection

$$
\operatorname{Ext}_{\text {Crys }^{+}(\mathbb{F})}^{1}\left(M_{W(\mathbb{F})}, M_{W(\mathbb{F})}\right) \otimes \mathbb{Q}_{p} / \mathbb{Z}_{p} \rightarrow \underset{n}{\lim } \operatorname{Ext}_{\mathcal{R}_{p^{n}}^{+}}^{1}\left(M^{\left(p^{n}\right)}, N^{\left(p^{n}\right)}\right),
$$

and (ii) implies that the first group is zero.

To prove (i), note that an element of either group splits when regarded as an extension of $W$-modules, and is therefore determined by the $\sigma$-linear map describing the action of the Frobenius. Since every $\sigma$-linear map $M^{\left(p^{n}\right)} \rightarrow N^{\left(p^{n}\right)}$ lifts to a $\sigma$ linear map $M \rightarrow N$, (i) is clear. The category $\operatorname{Isoc}^{+}\left(k^{\text {al }}\right)$ is semisimple (see 1.9), which implies (ii).

Lemma 7.13. When $k=\mathbb{F}$ or $\mathbb{F}_{q}$, every object in $\mathcal{R}^{+}\left(k ; \mathbb{Z}_{p}\right)$ is a quotient of a torsion-free object.

Proof. Suppose that the object $M$ is an extension of objects $M^{\prime}$ and $M^{\prime \prime}$ each of which is a quotient of a torsion-free object, say, $N^{\prime} \rightarrow M^{\prime}, N^{\prime \prime} \rightarrow M^{\prime \prime}$. Pull back the original extension by $N^{\prime \prime} \rightarrow M^{\prime \prime}$ :

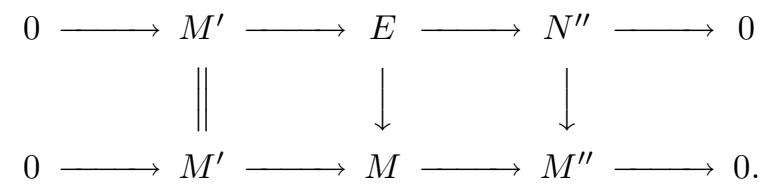

If the class of $E$ in $\operatorname{Ext}^{1}\left(N^{\prime \prime}, M^{\prime}\right)$ is the image of an element of $\operatorname{Ext}^{1}\left(N^{\prime \prime}, N^{\prime}\right)$, then $M$ also is a quotient of a torsion-free object. The obstruction to this is an element of $\operatorname{Ext}^{2}\left(N^{\prime \prime}, K\right)$ where $K$ is the kernel of $N^{\prime} \rightarrow M^{\prime}$. Certainly, when $k=\mathbb{F}_{q}$, this group will be zero if there exists a resolution

$$
0 \rightarrow A \stackrel{\cdot \lambda}{\longrightarrow} A \rightarrow N^{\prime \prime} \rightarrow 0
$$

where $A=W[F, \sigma]$ (because Exts in $\mathcal{R}^{+}\left(\mathbb{F}_{q} ; \mathbb{Z}_{p}\right)$ agree with those in $\operatorname{Mod}(A)$; this is explained on p. 541).

It suffices to prove the lemma in the case $k=\mathbb{F}_{q}$, and, for a given $M$, an argument using restriction of scalars (see the proof of Lemma 6.12) allows us to replace $\mathbb{F}_{q}$ by a finite extension. After such an extension, a torsion $M$ will have a composition 
series whose quotients are isomorphic to one of $(k, \sigma)$ or $(k, 0)$. Each of these is a quotient

$$
\begin{aligned}
& 0 \quad \rightarrow \quad(W, \sigma) \stackrel{p}{\rightarrow}(W, \sigma) \quad \rightarrow \quad(k, \sigma) \quad \rightarrow \quad 0, \\
& 0 \rightarrow(W, p \sigma) \stackrel{p}{\rightarrow}(W, p \sigma) \rightarrow(k, 0) \rightarrow 0 .
\end{aligned}
$$

Since $(W, \sigma) \cong A / A(F-\sigma)$ and $(W, p \sigma) \cong A / A(F-p \sigma)$ the above remarks prove the lemma for $M$. Let $M$ be an arbitrary object, and let $M_{1}=M / M_{\text {tors }}$. The obstruction to extending the statement from $M_{\text {tors }}$ and $M_{1}$ to $M$ is a torsion element of $\operatorname{Ext}^{2}\left(M_{1}, K\right)$, which by (7.12) becomes zero after a finite extension of $\mathbb{F}_{q}$.

Proposition 7.14. Let $\Gamma=\operatorname{Gal}\left(\mathbb{F} / \mathbb{F}_{q}\right)$, and let $M \mapsto \bar{M}$ denote the functor $\mathcal{R}^{+}\left(\mathbb{F}_{q} ; \mathbb{Z}_{p}\right) \rightarrow \mathcal{R}^{+}\left(\mathbb{F} ; \mathbb{Z}_{p}\right)$. For $M, N$ in $\mathcal{R}^{+}\left(\mathbb{F}_{q} ; \mathbb{Z}_{p}\right)$, there is a spectral sequence

$$
H^{r}\left(\Gamma, \operatorname{Ext}_{\mathcal{R}^{+}\left(\mathbb{F} ; \mathbb{Z}_{p}\right)}^{s}(\bar{M}, \bar{N})\right) \Longrightarrow \operatorname{Ext}_{\mathcal{R}^{+}\left(\mathbb{F}_{q} ; \mathbb{Z}_{p}\right)}^{r+s}(M, N) .
$$

Proof. Lemma 7.13 shows that $M$ has a flat resolution, and so this follows from Corollary 7.9 ,

Proposition 7.15. Let $\Gamma=\operatorname{Gal}\left(\mathbb{F} / \mathbb{F}_{q}\right)$. For $M, N$ in $\mathcal{R}^{+}\left(\mathbb{F}_{q} ; \mathbb{Z}_{p}\right)$, there is a spectral sequence

$$
H_{\text {cts }}^{r}\left(\Gamma, \operatorname{Ext}_{\text {Crys }^{+}(\mathbb{F})}^{s}\left(M_{W(\mathbb{F})}, N_{W(\mathbb{F})}\right)\right) \Longrightarrow \operatorname{Ext}_{\mathcal{R}^{+}\left(\mathbb{F}_{q} ; \mathbb{Z}_{p}\right)}^{r+s}(M, N) .
$$

Here $H_{\mathrm{cts}}^{r}$ is computed using continuous cochains relative to the p-adic topology on $\operatorname{Ext}_{W(\mathbb{F})}^{s}(M, N)$.

Proof. When $N$ is finite, the same argument as in the proof of Proposition 7.11 shows that (19) coincides with (18). We will obtain the general case by passing to the inverse limit in

$$
H_{\text {cts }}^{r}\left(\Gamma, \operatorname{Ext}_{\mathbf{C r y s}^{+}(\mathbb{F})}^{s}\left(M_{W(\mathbb{F})}, N_{W(\mathbb{F})}^{\left(p^{n}\right)}\right)\right) \Longrightarrow \operatorname{Ext}_{\mathcal{R}^{+}\left(\mathbb{F}_{q} ; \mathbb{Z}_{p}\right)}^{r+s}\left(M, N^{\left(p^{n}\right)}\right) .
$$

For the argument in the proof of (17.11) to apply, we must verify the following:

$$
\begin{aligned}
& \overleftarrow{n}_{n}^{\lim _{n}} N^{\left(p^{n}\right)}= \begin{cases}N & \text { if } i=0, \\
0 & \text { otherwise, } \quad N \text { in } \mathcal{R}^{+}\left(\mathbb{F}_{q} ; \mathbb{Z}_{p}\right) ;\end{cases} \\
& \stackrel{\lim }{n}^{i} \operatorname{Ext}_{\mathcal{R}^{+}\left(\mathbb{F}_{q} ; \mathbb{Z}_{p}\right)}\left(M, N^{\left(p^{n}\right)}\right)=0 \text { for } i \neq 0, \quad M, N \text { in } \mathcal{R}^{+}\left(\mathbb{F}_{q} ; \mathbb{Z}_{p}\right) ; \\
& \stackrel{\lim }{n}^{i} \operatorname{Ext}_{\text {Crys }^{+}(\mathbb{F})}^{s}\left(M, N^{\left(p^{n}\right)}\right)=0 \text { for } i \neq 0, \quad M, N \text { in } \operatorname{Crys}^{+}(\mathbb{F}) .
\end{aligned}
$$

Certainly, $N=\varliminf_{\varliminf} N^{\left(p^{n}\right)}$, and $\lim ^{i} N^{\left(p^{n}\right)}=0$ for $i>0$ because $N^{\left(p^{n}\right)}$ is of finite length over $W$ and we can apply [27, 7.2].

The second equality follows from the finiteness of $\operatorname{Ext}_{\mathcal{R}^{+}\left(\mathbb{F}_{q} ; \mathbb{Z}_{p}\right)}\left(M, N^{\left(p^{n}\right)}\right)$.

We now prove the third equality. If $p^{a}$ kills the torsion in $N$, then $p^{a} N$ is torsion-free, and there are exact sequences

$$
0 \rightarrow \operatorname{Ext}^{i}\left(M, p^{a} N\right)^{\left(p^{n}\right)} \rightarrow \operatorname{Ext}^{i}\left(M,\left(p^{a} N\right)^{\left(p^{n}\right)}\right) \rightarrow \operatorname{Ext}^{i+1}\left(M, p^{a} N\right)_{p^{n}} \rightarrow 0 .
$$

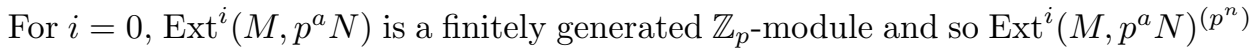
is finite; therefore $\lim ^{1}\left(\operatorname{Ext}^{i}\left(M, p^{a} N\right)^{\left(p^{n}\right)}\right)=0$. For $i>0, \operatorname{Ext}^{i}\left(M, p^{a} N\right)$ is torsion (recall that $\operatorname{Crys}^{+}(\mathbb{F})_{\mathbb{Q}} \simeq \operatorname{Isoc}^{+}(\mathbb{F})$ is semisimple), and, in fact, killed by a 
fixed power of $p$, and $\mathrm{se}^{12} \lim ^{1}\left(\operatorname{Ext}^{i}\left(M, p^{a} N\right)_{p^{n}}\right)=0$. The sequence shows that $\lim ^{1}\left(\operatorname{Ext}^{i}\left(M,\left(p^{a} N\right)^{\left(p^{n}\right)}\right)\right)=0$, and one can use the exact sequence

$$
0 \rightarrow N_{p^{a}} \rightarrow N \rightarrow p^{a} N \rightarrow 0
$$

to deduce that $\lim ^{1}\left(\operatorname{Ext}^{i}\left(M, N^{\left(p^{n}\right)}\right)\right)=0$.

\section{HomologicAl Algebra IN THE CATEGORY OF MOTIVES}

Throughout this section $k=\mathbb{F}_{q}$ or $\mathbb{F}$, and we assume that the $l$-adic Tate conjectures hold for $\mathcal{M}^{+}(k ; \mathbb{Q})$, i.e., that the maps (6) are isomorphisms.

Theorem 8.1. Let $X$ and $Y$ be effective motives in $\mathcal{M}^{+}(k ; \mathbb{Z})$.

(a) The map $(\alpha, \beta) \mapsto \alpha-\beta$,

$\operatorname{Hom}_{\mathcal{M}^{+}(k ; \mathbb{Q})}\left(X_{0}, Y_{0}\right) \times \operatorname{Hom}_{\mathcal{R}^{+}(k ; \hat{\mathbb{Z}})}\left(X_{f}, Y_{f}\right) \rightarrow \operatorname{Hom}_{\mathcal{R}^{+}\left(k ; \mathbb{A}_{f}\right)}\left(\omega_{f}\left(X_{0}\right), \omega_{f}\left(Y_{0}\right)\right)$,

is surjective with kernel $\operatorname{Hom}_{\mathcal{M}^{+}(k ; \mathbb{Z})}(X, Y)$.

(b) For $i>0, \operatorname{Ext}_{\mathcal{M}^{+}(k ; \mathbb{Z})}^{i}(X, Y)$ is torsion and the sequence

$$
0 \rightarrow \operatorname{Ext}_{\mathcal{M}^{+}(k ; \mathbb{Z})}^{i}(X, Y)_{\mathbb{Z}_{l}} \rightarrow \operatorname{Ext}_{\mathcal{R}\left(k ; \mathbb{Z}_{l}\right)}^{i}\left(X_{l}, Y_{l}\right) \rightarrow T_{l} \operatorname{Ext}_{\mathcal{M}^{+}(k ; \mathbb{Z})}^{i+1}(X, Y) \rightarrow 0
$$

is exact (all l); hence,

$$
\operatorname{Ext}_{\mathcal{M}^{+}(k ; \mathbb{Z})}^{i}(X, Y) \cong \operatorname{Ext}_{\mathcal{R}^{+}(k ; \hat{\mathbb{Z}})}^{i}\left(X_{f}, Y_{f}\right)_{\text {tors }} .
$$

(c) If $k=\mathbb{F}$, then $\operatorname{Ext}_{\mathcal{M}^{+}(k ; \mathbb{Z})}^{i}(X, Y)=0$ for all $i>2$, and for all $i>1$ when $X$ and $Y$ are torsion-free.

Proof. For any $\mathbb{Z}$-module $M$ of finite rank, the sequence

$$
0 \rightarrow M \rightarrow M_{\hat{\mathbb{Z}}} \times M_{\mathbb{Q}} \rightarrow M_{\mathbb{A}_{f}} \rightarrow 0
$$

is exact. We obtain (a) by taking $M=\operatorname{Hom}(X, Y)$ in this sequence and applying (5.6, 5.8, 5.10).

The remainder of the proof will require several steps.

$\operatorname{Ext}_{\mathcal{M}^{+}(k ; \mathbb{Z})}^{i}(X, Y)$ Is TORsion FOR $i>0$ : It suffices to prove that $\operatorname{Ext}_{\mathcal{M}^{+}(k ; \mathbb{Z})}^{1}(X, Y)$ is torsion. Moreover, we may assume that $X$ and $Y$ are torsion-free. Consider an exact sequence

$$
0 \rightarrow Y \rightarrow E \stackrel{\pi}{\rightarrow} X \rightarrow 0
$$

in $\mathcal{M}^{+}(k ; \mathbb{Z})$. Because $\mathcal{M}^{+}(k ; \mathbb{Q})$ is semisimple, there exists a morphism $\alpha_{0}: X_{0} \rightarrow$ $E_{0}$ such that $\pi_{0} \circ \alpha_{0}=\mathrm{id}_{X_{0}}$. For some $m \in \mathbb{Z}, m \alpha_{0} \in \operatorname{Hom}_{\mathcal{M}^{+}(k ; \mathbb{Z})}(X, E)$ (see 5.5). Now the equation $\pi \circ\left(m \alpha_{0}\right)=m$ id $_{X}$ implies that the class of the sequence is killed by $m$.

Write $f^{i}(X, Y)$ for the natural map

$$
\operatorname{Ext}_{\mathcal{M}^{+}(k ; \mathbb{Z})}^{i}(X, Y)_{\mathbb{Z}_{l}} \rightarrow \operatorname{Ext}_{\mathcal{R}^{+}\left(k ; \mathbb{Z}_{l}\right)}^{i}\left(X_{l}, Y_{l}\right) .
$$

Recall (5.10) that $f^{0}(X, Y)$ is an isomorphism.

\footnotetext{
${ }^{12}$ Recall that an inverse system of abelian groups $\left(A_{n}\right)_{n \in \mathbb{N}}$ is said to satisfy the Mittag-Leffler condition if, for each $n$, the decreasing chain in $A_{n}$ of the images of the $A_{m}$ for $m>n$ is eventually constant, and that the higher inverse limits vanish for such a system. In the case $i=0$, the condition obviously holds, and in the case $i>0$ the images are eventually zero.

In the text we are implicitly using that for inverse systems indexed by $\mathbb{N}$, only the first higher inverse limit can be nonzero [46].
} 
The maps $f^{i}(X, Y)$ ARe isomorphisms when $X$ And $Y$ ARe torsion. The functor $X \mapsto X_{f}$ defines an equivalence of the category of torsion effective motives with the category of torsion objects in $\mathcal{R}^{+}(k ; \hat{\mathbb{Z}})$, and so it suffices to prove that the Exts in the torsion subcategories coincide with the Exts in the full categories. We do this for $\mathcal{M}^{+}(k ; \mathbb{Z})$ since $\mathcal{R}^{+}\left(k ; \mathbb{Z}_{l}\right)$ is similar. Because $\mathcal{M}^{+}(k ; \mathbb{Z})$ is noetherian, $\operatorname{Ind}\left(\mathcal{M}^{+}(k ; \mathbb{Z})\right)$ has enough injectives, and the Exts computed using injective resolutions coincide with the Yoneda Exts (7.1). It follows from (6.12b) that every injective object in $\operatorname{Ind}\left(\mathcal{M}^{+}(k ; \mathbb{Z})\right)$ is divisible, and so an injective resolution $Y \rightarrow I^{\bullet}$ in $\operatorname{Ind}\left(\mathcal{M}^{+}(k ; \mathbb{Z})\right)$ gives an injective resolution $Y \rightarrow I_{\text {tors }}^{\bullet}$ in the subcategory of torsion objects. As $\operatorname{Hom}\left(X, I_{\text {tors }}^{\bullet} \cong \operatorname{Hom}\left(X, I^{\bullet}\right)\right.$, this proves that the Exts in the two categories agree.

The maps $f^{i}(X, Y)$ ARE ISOMORPhisms When $Y$ Is TORSiOn. It suffices to prove this when $X$ is torsion-free and $Y$ is $l$-torsion, say $l^{n} Y=0$. The Ext sequences of

$$
0 \rightarrow X \stackrel{l^{n}}{\rightarrow} X \rightarrow X / l^{n} X \rightarrow 0
$$

give a diagram

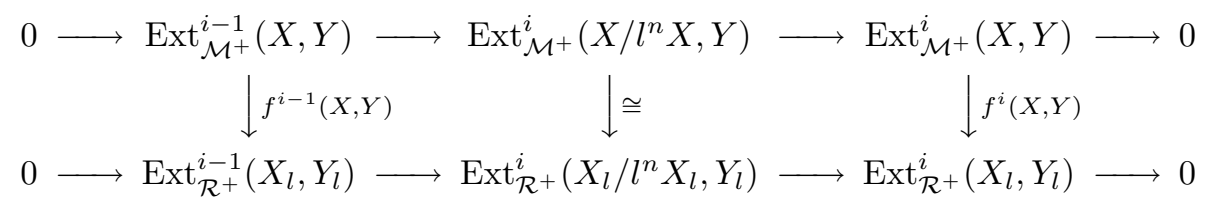

from which it follows that $f^{i-1}(X, Y)$ is injective and $f^{i}(X, Y)$ is surjective. Since this holds for all $i, f^{i}(X, Y)$ is an isomorphism.

The maps $f^{i}(X, Y)$ are isomorphisms when $X$ is torsion. Similar to the preceding case.

The SEQUenCE (20) IS EXACT. Because $f^{i}(X, Y)$ is an isomorphism when $Y$ is torsion, $f^{i}\left(X, Y^{\bullet}\right)$ (map of hyper-Exts) is an isomorphism when $Y^{\bullet}$ is a bounded complex with finite cohomology, for example, when $Y^{\bullet}=Y^{\left\{l^{n}\right\}}=\mathrm{df}_{\mathrm{df}} Y \stackrel{l^{n}}{\longrightarrow} Y$ :

$$
\operatorname{Ext}_{\mathcal{M}^{+}}^{i}\left(X, Y^{\left\{l^{n}\right\}}\right) \cong \operatorname{Ext}_{\mathcal{R}^{+}}^{i}\left(X_{l}, Y_{l}^{\left\{l^{n}\right\}}\right) \text {. }
$$

There are exact sequences

$$
0 \rightarrow \operatorname{Ext}_{\mathcal{M}^{+}}^{i}(X, Y)^{\left(l^{n}\right)} \rightarrow \operatorname{Ext}_{\mathcal{M}^{+}}^{i}\left(X, Y^{\left\{l^{n}\right\}}\right) \rightarrow \operatorname{Ext}_{\mathcal{M}^{+}}^{i}(X, Y)_{l^{n}} \rightarrow 0
$$

and

$$
\begin{aligned}
\lim _{\longleftarrow} \operatorname{Ext}_{\mathcal{M}^{+}}^{i}\left(X, Y^{\left\{l^{n}\right\}}\right) \cong \lim _{\longleftarrow} \operatorname{Ext}_{\mathcal{R}^{+}}^{i}\left(X_{l}, Y_{l}^{\left\{l^{n}\right\}}\right) & \cong \lim _{\longleftarrow} \operatorname{Ext}_{\mathcal{R}^{+}}^{i}\left(X_{l}, Y_{l}^{\left(l^{n}\right)}\right) \\
& \cong \operatorname{Ext}_{\mathcal{R}^{+}}^{i}\left(X_{l}, Y_{l}\right)
\end{aligned}
$$

(for the third isomorphism, see the proofs of 7.11 and 7.15). Thus, on passing to the inverse limit in (22), we obtain (20).

This completes the proof of (b). We now assume that $k=\mathbb{F}$.

$\operatorname{Ext}_{\mathcal{M}^{+}}^{2}(X, Y)=0$ IF $X$ ANd $Y$ ARE TORSION-FREE. For an integer $m$, let $\mathcal{M}_{m}^{+}$ (resp. $\left.\mathcal{R}_{m}^{+}\right)$denote the subcategory of $\mathcal{M}^{+}(k ; \mathbb{Z})\left(\right.$ resp. $\left.\mathcal{R}^{+}(k ; \hat{\mathbb{Z}})\right)$ of objects killed by $m$. On passing to the direct limit in the sequence

$$
0 \rightarrow \operatorname{Ext}_{\mathcal{M}^{+}}^{1}(X, Y)^{(m)} \rightarrow \operatorname{Ext}_{\mathcal{M}^{+}}^{1}\left(X, Y^{(m)}\right) \rightarrow \operatorname{Ext}_{\mathcal{M}^{+}}^{2}(X, Y)_{m} \rightarrow 0
$$


and using that $\operatorname{Ext}_{\mathcal{M}^{+}}^{1}(X, Y)$ and $\operatorname{Ext}_{\mathcal{M}^{+}}^{2}(X, Y)$ are torsion, we find that

$$
\underset{m}{\lim _{m}} \operatorname{Ext}_{\mathcal{M}^{+}}^{1}\left(X, Y^{(m)}\right) \cong \operatorname{Ext}_{\mathcal{M}^{+}}^{2}(X, Y) .
$$

Consider the diagram

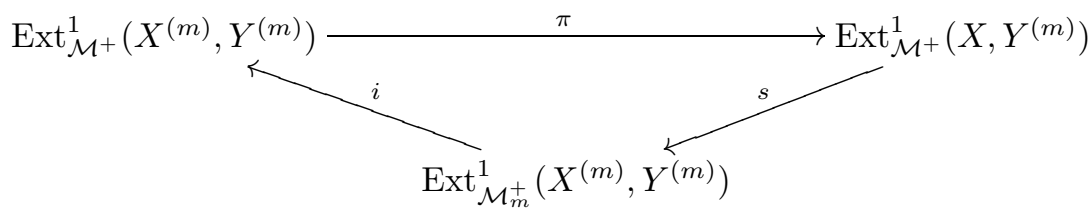

in which $\pi$ is the surjection induced by $X \rightarrow X^{(m)}, i$ is the obvious injection, and $s$ applied to a short exact sequence replaces each motive $E$ in the sequence with $E^{(m)}$. One checks easily that $s \circ(\pi \circ i)=\mathrm{id}$ and $(\pi \circ i) \circ s=\mathrm{id}$, and so $s$ is an isomorphism. Thus,

$$
\operatorname{Ext}_{\mathcal{M}^{+}}^{2}(X, Y) \cong \underset{m}{\lim _{m}} \operatorname{Ext}_{\mathcal{M}_{m}^{+}}^{1}\left(X^{(m)}, Y^{(m)}\right) \cong \underset{m}{\lim _{m}} \operatorname{Ext}_{\mathcal{R}_{m}^{+}}^{1}\left(X_{f}^{(m)}, Y_{f}^{(m)}\right) .
$$

For $l \neq p, \operatorname{Ext}_{\mathcal{R}_{l^{n}}^{+}}^{1}\left(X_{f}^{\left(l^{n}\right)}, Y_{f}^{\left(l^{n}\right)}\right)$ is obviously zero, and so it remains to show that

$$
\underset{p^{n}}{\lim _{\longrightarrow}} \operatorname{Ext}_{\mathcal{R}_{p^{n}}^{+}}^{1}\left(X_{p}^{\left(p^{n}\right)}, Y_{p}^{\left(p^{n}\right)}\right)=0
$$

But this is shown in the proof of (7.12).

$\operatorname{Ext}_{\mathcal{M}^{+}}^{i}(X, Y)=0$ FOR ALL $i>2$. This follows from (b) and the fact that $\operatorname{Ext}_{\mathcal{R}^{+}\left(k ; \mathbb{Z}_{l}\right)}^{i}\left(X_{l}, Y_{l}\right)=0$ for $i>2$ (for $l \neq p$, this is obvious, and for $l=p$ it can be proved by the arguments used to prove the $p$-case of Theorem 9.1 below).

Remark 8.2. The group $\operatorname{Ext}_{\mathcal{R}^{+}\left(\mathbb{F} ; \mathbb{Z}_{\ell}\right)}(M, N)$ need not be torsion. Consider, for example, the extension

$$
0 \rightarrow \mathbb{Z}_{\ell} \rightarrow \mathbb{Z}_{\ell} \oplus \mathbb{Z}_{\ell} \rightarrow \mathbb{Z}_{\ell} \rightarrow 0
$$

on which the Frobenius element in $\Gamma=\operatorname{Gal}\left(\mathbb{F} / \mathbb{F}_{p}\right)$ acts on the middle term as the matrix $\left(\begin{array}{ll}1 & a \\ 0 & 1\end{array}\right)$ with $a$ a nonzero element of $\mathbb{Z}_{\ell}$. Multiplying the class of the extension by $m$ corresponds to multiplying $a$ by $m$, and so this represents a nontorsion element of $\operatorname{Ext}_{\mathcal{R}^{+}\left(\mathbb{F}_{p} ; \mathbb{Z}_{\ell}\right)}^{1}(M, N)$, which remains nontorsion in $\operatorname{Ext}_{\mathcal{R}^{+}\left(\mathbb{F} ; \mathbb{Z}_{\ell}\right)}^{1}(M, N)$. Thus, in general,

$$
\operatorname{Ext}_{\mathcal{M}^{+}(\mathbb{F} ; \mathbb{Z})}^{1}(X, Y)(\ell) \not \approx \operatorname{Ext}_{\mathcal{R}\left(\mathbb{F} ; \mathbb{Z}_{\ell}\right)}^{1}\left(X_{\ell}, Y_{\ell}\right)
$$

Similarly, the group $\operatorname{Ext}_{\mathcal{R}^{+}\left(\mathbb{F} ; \mathbb{Z}_{p}\right)}^{1}(M, N)$ need not be torsion (because the category $\mathcal{R}^{+}\left(\mathbb{F} ; \mathbb{Z}_{p}\right)$, in contrast to $\mathbf{I s o c}^{+}(\mathbb{F})$, is not semisimple).

Lemma 8.3. Every effective motive over $\mathbb{F}_{q}$ or $\mathbb{F}$ is a quotient of a torsion-free effective motive.

Proof. It suffices to prove the lemma in the case $k=\mathbb{F}_{q}$, and, for a given $X$, an argument using restriction of scalars allows us to replace $\mathbb{F}_{q}$ by a finite extension (see the proof of $(6.12 \mathrm{~b})$ ).

After such an extension, a $p$-torsion object $X$ will have a composition series whose quotients are isomorphic to one of $(k, \sigma)$ or $(k, 0)$. But $(k, \sigma) \cong \mathbb{1} / p \mathbb{1}$ and $(k, 0) \cong \mathbb{L} / p \mathbb{L}$, and so the same argument as in the proof of Lemma 7.13 (using (21) ) proves the lemma for $X$. 
Let $X$ be an arbitrary effective motive, and let $X_{1}=X / X_{p \text {-tors. }}$ For $X_{1}$, the same argument as in the proof of (6.12) proves the lemma. Let $Y \rightarrow X_{p \text {-tors }}$ be a surjective map with $Y$ torsion-free, and let $K$ be its kernel. The obstruction to extending the lemma from $X_{1}$ and $X / X_{p \text {-tors }}$ to $X$ is a torsion class in $\operatorname{Ext}^{2}\left(X_{1}, K\right)$ (see the proof of 7.13), but this vanishes by (20) and (7.12).

Theorem 8.4. Let $\Gamma=\operatorname{Gal}\left(\mathbb{F} / \mathbb{F}_{q}\right)$. For all motives $X$ and $Y$ over $\mathbb{F}_{q}$, there is a spectral sequence:

$$
H^{r}\left(\Gamma, \operatorname{Ext}_{\mathcal{M}^{+}(\mathbb{F} ; \mathbb{Z})}^{s}\left(X_{/ \mathbb{F}}, Y_{/ \mathbb{F}}\right)\right) \Longrightarrow \operatorname{Ext}_{\mathcal{M}^{+}\left(\mathbb{F}_{q} ; \mathbb{Z}\right)}^{r+s}(X, Y) .
$$

Proof. Lemma 8.3 shows that $X$ has a flat resolution. Now apply Corollary [7.9] as in the proof of 7.14

\section{Examples.}

Example 8.5. We apply (8.1) to compute $\operatorname{Ext}_{\mathcal{M}^{+}(\mathbb{F} ; \mathbb{Z})}(\mathbb{Z}, \mathbb{Z}(r)$ ) (we are writing $\mathbb{Z}$ for $\mathbb{1}$ and $\mathbb{Z}(r)$ for $\left.\mathbb{T}^{\otimes r}\right)$.

For $r=0, \operatorname{Hom}_{\mathcal{M}^{+}(\mathbb{F} ; \mathbb{Z})}(\mathbb{Z}, \mathbb{Z}(0)) \cong \mathbb{Z}$ and $\operatorname{Ext}_{\mathcal{M}^{+}(\mathbb{F} ; \mathbb{Z})}(\mathbb{Z}, \mathbb{Z}(0))=0$ for $i=1,2$.

For $r \neq 0, \operatorname{Hom}_{\mathcal{M}^{+}(\mathbb{F} ; \mathbb{Z})}(\mathbb{Z}, \mathbb{Z}(r))=0$, and so

$$
\operatorname{Hom}_{\mathcal{M}+(\mathbb{F} ; \mathbb{Z})}\left(\mathbb{Z}, \mathbb{Z}(r)^{(m)}\right) \stackrel{\cong}{\rightrightarrows} \operatorname{Ext}_{\mathcal{M}^{+}(\mathbb{F} ; \mathbb{Z})}^{1}(\mathbb{Z}, \mathbb{Z}(r))_{m}
$$

For $m=p^{n}$, the left-hand side is zero, and for $m=\ell^{n}$ it is $\left(\mathbb{Z}^{\left(\ell^{n}\right)}\right)(r)$. Therefore, on passing to the direct limit over $m$, we find that

$$
\operatorname{Ext}_{\mathcal{M}^{+}(\mathbb{F} ; \mathbb{Z})}^{1}(\mathbb{Z}, \mathbb{Z}(r)) \cong\left(\bigoplus_{\ell \neq p} \mathbb{Q}_{\ell} / \mathbb{Z}_{\ell}\right)(r)
$$

where $\left(\mathbb{Q}_{\ell} / \mathbb{Z}_{\ell}\right)(r)$ is $\mathbb{Q}_{\ell} / \mathbb{Z}_{\ell}$ with the Frobenius element of $\operatorname{Gal}\left(\mathbb{F} / \mathbb{F}_{q}\right)$ acting as $q^{r}$. Moreover, $\operatorname{Ext}_{\mathcal{M}+(\mathbb{F}, \mathbb{Z})}^{i}(\mathbb{Z}, \mathbb{Z}(r))=0$ for $i \neq 0,2$. (Without the + , this statement holds only modulo $p$-torsion.)

Example 8.6. We compute $\operatorname{Ext}_{\mathcal{M}^{+}\left(\mathbb{F}_{q}, \mathbb{Z}\right)}^{1}(\mathbb{Z}, \mathbb{Z}(r))$ for $r>0$. The spectral sequence in (8.4) shows that $\operatorname{Ext}_{\mathcal{M}^{+}\left(\mathbb{F}_{q}, \mathbb{Z}\right)}(\mathbb{Z}, \mathbb{Z}(r))$ and $\operatorname{Ext}_{\mathcal{M}^{+}\left(\mathbb{F}_{q}, \mathbb{Z}\right)}^{2}(\mathbb{Z}, \mathbb{Z}(r))$ are the kernel and cokernel respectively of

$$
\bigoplus_{\ell \neq p} \mathbb{Q}_{\ell} / \mathbb{Z}_{\ell} \stackrel{x \mapsto\left(q^{r}-1\right) x}{\longrightarrow} \bigoplus_{\ell \neq p} \mathbb{Q}_{\ell} / \mathbb{Z}_{\ell}
$$

Therefore $\operatorname{Ext}_{\mathcal{M}^{+}\left(\mathbb{F}_{q} ; \mathbb{Z}\right)}^{1}(\mathbb{Z}, \mathbb{Z}(r))$ is cyclic of order $q^{r}-1$, and $\operatorname{Ext}_{\mathcal{M}^{+}\left(\mathbb{F}_{q} ; \mathbb{Z}\right)}(\mathbb{Z}, \mathbb{Z}(r))=$ 0 for $i \neq 0,2$.

Example 8.7. Let $A$ be an abelian variety over $\mathbb{F}$, and let $h_{1} A$ be the isomotive $h_{1}(A)_{0}$ endowed with the $\mathbb{Z}$-structure provided by the maps $H_{1}\left(A, \mathbb{Z}_{l}\right) \rightarrow H_{1}\left(A, \mathbb{Q}_{l}\right)$. Since $\operatorname{Hom}\left(\mathbb{Z}, h_{1} A\right)=0$,

$$
\operatorname{Hom}_{\mathcal{M}^{+}(\mathbb{F} ; \mathbb{Z})}\left(\mathbb{Z},\left(h_{1} A\right)^{(m)}\right) \cong \operatorname{Ext}_{\mathcal{M}^{+}(\mathbb{F} ; \mathbb{Z})}^{1}\left(\mathbb{Z}, h_{1} A\right)_{m}
$$

for all $m$, and so

$$
\operatorname{Hom}_{\mathcal{M}^{+}(\mathbb{F} ; \mathbb{Z})}\left(\mathbb{Z}, h_{1} A \otimes \mathbb{Q} / \mathbb{Z}\right) \cong \operatorname{Ext}_{\mathcal{M}^{+}(\mathbb{F} ; \mathbb{Z})}^{1}\left(\mathbb{Z}, h_{1} A\right) .
$$

But

$$
\operatorname{Hom}_{\mathcal{M}^{+}(\mathbb{F} ; \mathbb{Z})}\left(\mathbb{Z}, h_{1} A \otimes \mathbb{Q} / \mathbb{Z}\right) \cong \operatorname{Hom}_{\mathcal{R}^{+}(\mathbb{F} ; \hat{\mathbb{Z}})}\left(\mathbb{Z},\left(h_{1} A\right)_{f \mathbb{Q}} /\left(h_{1} A\right)_{f}\right) \cong A(\mathbb{F}) .
$$


Thus,

$$
\operatorname{Ext}_{\mathcal{M}^{+}(\mathbb{F} ; \mathbb{Z})}^{1}\left(\mathbb{Z}, h_{1} A\right) \cong A(\mathbb{F}) .
$$

When $A$ is defined over $\mathbb{F}_{q}, \operatorname{Gal}\left(\mathbb{F} / \mathbb{F}_{q}\right)$ acts, and, on taking invariants, we find that

$$
\operatorname{Ext}_{\mathcal{M}^{+}\left(\mathbb{F}_{q} ; \mathbb{Z}\right)}^{1}\left(\mathbb{Z}, h_{1} A\right) \cong A\left(\mathbb{F}_{q}\right) .
$$

A similar argument using that $\operatorname{Hom}_{\mathcal{M}^{+}(\mathbb{F} ; \mathbb{Z})}\left(\left(h_{1} A\right)(-1), \mathbb{Z}\right)=0$ shows that

$$
\operatorname{Hom}_{\mathcal{M}^{+}(\mathbb{F} ; \mathbb{Z})}\left(\left(h_{1} A\right)(-1), \mathbb{Q} / \mathbb{Z}\right) \cong \operatorname{Ext}_{\mathcal{M}^{+}(\mathbb{F} ; \mathbb{Z})}^{1}\left(\left(h_{1} A\right)(-1), \mathbb{Z}\right) .
$$

But

$$
\operatorname{Hom}_{\mathcal{M}^{+}(\mathbb{F} ; \mathbb{Z})}\left(\left(h_{1} A\right)(-1), \mathbb{Q} / \mathbb{Z}\right) \cong \operatorname{Hom}_{\mathcal{R}^{+}(\mathbb{F} ; \hat{\mathbb{Z}})}\left(\left(h_{1} A\right)_{f}(-1), \mathbb{Q} / \mathbb{Z}\right) \cong A^{\vee}(\mathbb{F})
$$

where $A^{\vee}$ is the dual abelian variety. For an abelian variety $A$ over $\mathbb{F}_{q}$, this becomes

$$
\operatorname{Ext}_{\mathcal{M}^{+}\left(\mathbb{F}_{q} ; \mathbb{Z}\right)}\left(\left(h_{1} A\right)(-1), \mathbb{Z}\right) \cong A^{\vee}\left(\mathbb{F}_{q}\right) .
$$

Remark 8.8. It is generally conjectured, that for a smooth variety $V$ over a field $F$,

$$
H_{\text {mot }}^{i}(V, \mathbb{Q}(r)) \cong\left(K_{2 r-i} V\right)_{\mathbb{Q}}^{(r)}
$$

and

$$
H_{\text {mot }}^{i}(V, \mathbb{Q}(r)) \cong \operatorname{Ext}_{\mathcal{M M}(F ; \mathbb{Q})}^{i}(\mathbb{1},(h V)(r)) ;
$$

see, for example, [9, 3.7, 3.3]. Here $\operatorname{Ext}_{\mathcal{M M}(F ; \mathbb{Q})}^{i}(\mathbb{1},(h V)(r))$ is computed in the conjectural category of mixed isomotives over $F$, and $\left(K_{2 r-i} V\right)_{\mathbb{Q}}^{(r)}$ is the subspace of $\left(K_{2 r-1} V\right)_{\mathbb{Q}}$ on which the Adams operators $\psi^{k}$ act as $k^{r}$.

In particular, for $F$ itself, it is conjectured that

$$
\operatorname{Ext}_{\mathcal{M M}(F ; \mathbb{Q})}^{1}(\mathbb{Q}, \mathbb{Q}(r)) \cong\left(K_{2 r-1} F\right)_{\mathbb{Q}}^{(r)} .
$$

For $F=\mathbb{F}_{q}$ or $\mathbb{F}$, the statement (27) is true because the $K$-groups are torsion and, under any definition, the category of mixed isomotives will be semisimple; so both sides are 0 .

With $\mathbb{Z}$-coefficients, statement (27) is expected to be false in general, and (25) is definitely false 13 However, Quillen 45 , shows that $K_{2 r-1} \mathbb{F} \approx \bigoplus_{\ell \neq p} \mathbb{Q}_{\ell} / \mathbb{Z}_{\ell}$ with the Frobenius automorphism acting as $p^{r}$ and $K_{2 r-1} \mathbb{F}_{q}=\left(K_{2 r-1} \mathbb{F}\right)^{\operatorname{Gal}\left(\mathbb{F} / \mathbb{F}_{q}\right)}$, and Hiller 23] and Kratzer 32 show that the Adams operator $\psi^{k}$ acts on $K_{2 r-1} \mathbb{F}$ as multiplication by $k^{r}$, and so an integral version of (27) does hold when $F=\mathbb{F}_{q}$ or $\mathbb{F}$ :

$$
\operatorname{Ext}_{\mathcal{M}^{+}(F ; \mathbb{Z})}^{1}(\mathbb{Z}, \mathbb{Z}(r)) \approx\left(K_{2 r-1} F\right)^{(r)} .
$$

The isomorphism (28) can be made canonical: Soulé [50, IV.2, p. 284] proves that the Chern class map $c_{i, 1}: K_{2 i-1}\left(\mathbb{F}_{q}\right)_{\mathbb{Z}_{l}} \rightarrow H^{1}\left(\mathbb{F}_{q}, \mathbb{Z}_{l}(i)\right)$ is an isomorphism if $i<l$; later Dwyer and Friedlander [12] defined a Chern character $c h_{i, 1}: K_{2 i-1}\left(\mathbb{F}_{q}\right)_{\mathbb{Z}_{l}} \rightarrow$ $H^{1}\left(\mathbb{F}_{q}, \mathbb{Z}_{l}(i)\right)$ that is related to the Chern class map by $(-1)^{i-1}(i-1) ! c h_{i, 1}=c_{i, 1}$ [51, 3.2.2, p. 267].

\footnotetext{
${ }^{13}$ For a projective smooth variety over a field $k$,

$$
H_{\operatorname{mot}}^{3}(V, \mathbb{Z}(1))=H^{2}\left(V, \mathbb{G}_{m}\right),
$$
}

which is a nonzero torsion group in general whereas $K_{-1} V=0$. See also Bloch and Esnault 5 , p. 304], which explains why a closely related conjecture should not be true integrally. 


\section{Extensions And Zeta Functions: LocAl CASE}

We use the notations: $\Gamma=\operatorname{Gal}\left(\mathbb{F} / \mathbb{F}_{q}\right), q=p^{a}, W=W\left(\mathbb{F}_{q}\right), B=B\left(\mathbb{F}_{q}\right)$, $A=W[F, \sigma], \mathcal{R}_{\ell}=\mathcal{R}\left(\mathbb{F}_{q} ; \mathbb{Z}_{\ell}\right)={ }_{\mathrm{df}} \mathcal{R}\left(\Gamma ; \mathbb{Z}_{\ell}\right), \overline{\mathcal{R}}_{\ell}=\operatorname{Mod}\left(\mathbb{Z}_{\ell}\right), \mathcal{R}_{p}=\mathcal{R}^{+}\left(\mathbb{F}_{q}, \mathbb{Z}_{p}\right)={ }_{\mathrm{df}}$ $\operatorname{Crys}^{+}\left(\mathbb{F}_{q}\right), \overline{\mathcal{R}}_{p}=\operatorname{Crys}^{+}(\mathbb{F})$.

Statement of the theorem. Let $M$ be an object of $\mathcal{R}_{l}$. The Frobenius endomorphism $\pi_{M}$ of $M$ is the map induced by the element $x \mapsto x^{q}$ of $\Gamma$ when $l \neq p$ and by $F^{a}$ when $l=p$. We denote the characteristic and minimum polynomials of $\pi_{M}$ acting on $M_{\mathbb{Q}_{l}}$ by $m_{M}(t)$ and $P_{M}(t)$, respectively. They are monic polynomials with coefficients in $\mathbb{Z}_{l}$ (see [11, p. 89] for the case $l=p$ ). For $M$ in $\mathcal{R}_{p}$, let $r(M)$ denote the rank of $M$ and $s(M)$ the sum of the slopes of $M$; thus if

$$
P_{M}(T)=t^{h}+\cdots+c
$$

then $r(M)=h$ and $s(M)=\operatorname{ord}_{p}(c) / a$ (cf. [11, p. 90]). For $M$ in $\mathcal{R}_{\ell}$ we set $s(M)=0$.

Let $M, N$ be objects of $\mathcal{R}_{l}$. From the spectral sequences $(\mathbf{7 . 1 1}$, 17.15) we obtain a map

$$
\operatorname{Hom}_{\overline{\mathcal{R}}_{l}}(\bar{M}, \bar{N})_{\Gamma} \rightarrow \operatorname{Ext}_{\mathcal{R}_{l}}^{1}(M, N)
$$

Define

$$
f=f(M, N): \operatorname{Hom}_{\mathcal{R}_{l}}(M, N) \rightarrow \operatorname{Ext}_{\mathcal{R}_{l}}^{1}(M, N)
$$

to be the composite of this with the obvious map $\operatorname{Hom}_{\mathcal{R}_{l}}(M, N) \rightarrow \operatorname{Hom}_{\overline{\mathcal{R}}_{l}}(\bar{M}, \bar{N})_{\Gamma}$.

Theorem 9.1. If $m_{M}(T)$ and $m_{N}(T)$ have no multiple root in common, then $z(f)$ is defined and satisfies

$$
z(f) \cdot\left[\operatorname{Ext}_{\mathcal{R}_{l}}^{2}(M, N)\right]=\left|q^{s(M) \cdot r(N)} \cdot \prod_{a_{i} \neq b_{j}}\left(1-\frac{b_{j}}{a_{i}}\right)\right|_{l}
$$

where $\left(a_{i}\right)_{1 \leq i \leq r(M)}$ and $\left(b_{j}\right)_{1 \leq j \leq r(N)}$ are the roots of $P_{M}(t)$ and $P_{N}(t)$, respectively.

Remark 9.2. (a) When $l \neq p$, the term $q^{s(M) \cdot r(N)}=1$.

(b) In the course of the proof, we shall show that $\operatorname{Ext}_{\mathcal{R}_{l}}^{i}(M, N)=0$ for $i>2$ (without any condition on $M$ and $N$ ) and that $\operatorname{Ext}_{\mathcal{R}_{l}}^{2}(M, N)$ is finite.

(c) When one of $M$ or $N$ is finite, the theorem simply states that the groups $\operatorname{Ext}_{\mathcal{R}_{l}}^{i}(M, N)$ are finite and the alternating product of their orders is 1 .

(d) Let

$$
P_{M^{\vee} \otimes N}(t)=\prod\left(1-\frac{b_{j}}{a_{i}} t\right),
$$

and let $\rho(M, N)$ be the rank of $\operatorname{Hom}_{\mathcal{R}_{l}}(M, N)$. Then $\rho(M, N)$ is the number of pairs $(i, j)$ with $a_{i}=b_{j}$, and (29) becomes the equation

$$
z(f) \cdot\left[\operatorname{Ext}^{2}(M, N)\right]=\left|q^{s(M) \cdot r(N)} \cdot \lim _{t \rightarrow 1} \frac{P_{M^{\vee} \otimes N}(t)}{(1-t)^{\rho(M, N)}}\right|_{l} .
$$

(e) When the groups $\operatorname{Ker}(f), \operatorname{Coker}(f), \operatorname{Ext}_{\mathcal{R}_{l}}^{2}(M, N), \operatorname{Ext}_{\mathcal{R}_{l}}^{3}(M, N), \ldots$ are finite and almost all zero, then we say that $\chi^{\times}(M, N)$ is defined, and we set it equal to the alternating product of the orders of the groups. Granted (b), Theorem 
9.1 is equivalent to the statement: if $m_{M}(T)$ and $m_{N}(T)$ have no multiple root in common, then $\chi^{\times}(M, N)$ is defined and equals

$$
\left|q^{s(M) \cdot r(N)} \cdot \prod_{a_{i} \neq b_{j}}\left(1-\frac{b_{j}}{a_{i}}\right)\right|_{l} .
$$

(f) Let

$$
0 \rightarrow N^{\prime} \rightarrow N \rightarrow N^{\prime \prime} \rightarrow 0
$$

be an exact sequence in $\mathcal{R}_{l}$. If $\chi^{\times}\left(M, N^{\prime}\right)$ and $\chi^{\times}\left(M, N^{\prime \prime}\right)$ are defined, then so also is $\chi^{\times}(M, N)$, and

$$
\chi^{\times}(M, N)=\chi^{\times}\left(M, N^{\prime}\right) \cdot \chi^{\times}\left(M, N^{\prime \prime}\right) .
$$

Since a similar statement holds for exact sequences in $M$ and the expression (30) is multiplicative, we see that it suffices to prove Theorem 9.1 for $M$ and $N$ running through a set of generators for the Grothendieck group $K\left(\mathcal{R}_{l}\right)$ of $\mathcal{R}_{l}$.

Even more is true: both $\chi^{\times}$and the expression in (30) take values in $\mathbb{Q}_{+}^{\times}$, the group of positive rational numbers, which is torsion-free. Therefore, in order to prove Theorem 9.1 it suffices to verify it for $M$ and $N$ running through a set of generators for $K\left(\mathcal{R}_{l}\right) \otimes \mathbb{Q}$.

Proof of Theorem 9.1 in the case $l \neq p$. From Proposition[7.11, we obtain an exact sequence and isomorphisms

$$
\begin{aligned}
& \operatorname{Hom}_{\mathcal{R}_{\ell}}(M, N) \cong \operatorname{Hom}_{\mathbb{Z}_{\ell}}(M, N)^{\Gamma}, \\
& 0 \rightarrow \operatorname{Hom}_{\mathbb{Z}_{\ell}}(M, N)_{\Gamma} \stackrel{c}{\rightarrow} \operatorname{Ext}_{\mathcal{R}_{\ell}}^{1}(M, N) \rightarrow \operatorname{Ext}_{\mathbb{Z}_{\ell}}^{1}(M, N)^{\Gamma} \rightarrow 0, \\
& \operatorname{Ext}_{\mathbb{Z}_{\ell}}^{1}(M, N)_{\Gamma} \cong \operatorname{Ext}_{\mathcal{R}_{l}}^{2}(M, N), \\
& \operatorname{Ext}_{\mathcal{R}_{l}}^{i}(M, N)=0 \text { for } i>2 .
\end{aligned}
$$

Let $f_{0}$ be the map

$$
\operatorname{Hom}_{\mathbb{Z}_{\ell}}(M, N)^{\Gamma} \rightarrow \operatorname{Hom}_{\mathbb{Z}_{\ell}}(M, N)_{\Gamma}
$$

induced by the identity map. Then $f=c \circ f_{0}$, and so (see 9.9)

$$
z(f)=z\left(f_{0}\right) /\left[\operatorname{Ext}_{\mathbb{Z}_{\ell}}^{1}(M, N)^{\Gamma}\right] .
$$

Let $\gamma$ be the generator $x \mapsto x^{q}$ of $\Gamma$. The family of eigenvalues of $\gamma$ on $\operatorname{Hom}_{\mathbb{Z}_{\ell}}(M, N)$ is $\left(\frac{b_{i}}{a_{j}}\right)_{i, j}$. Because of our hypothesis on $m_{M}(t)$ and $m_{N}(t)$, (9.10) applies, and shows that

$$
z\left(f_{0}\right)=\left|\prod_{a_{i} \neq b_{j}}\left(1-\frac{b_{i}}{a_{j}}\right)\right|_{\ell} .
$$

Because $\operatorname{Ext}_{\mathbb{Z}_{\ell}}^{1}(M, N)$ is finite,

$$
\left[\operatorname{Ext}_{\mathbb{Z}_{\ell}}^{1}(M, N)^{\Gamma}\right]=\left[\operatorname{Ext}_{\mathbb{Z}_{\ell}}^{1}(M, N)_{\Gamma}\right]
$$

and so

$$
\left[\operatorname{Ext}_{\mathcal{R}_{l}}^{2}(M, N)\right]=\left[\operatorname{Ext}_{\mathbb{Z}_{\ell}}^{1}(M, N)^{\Gamma}\right] .
$$

On substituting for $z\left(f_{0}\right)$ and $\left[\operatorname{Ext}_{\mathbb{Z}_{\ell}}^{1}(M, N)^{\Gamma}\right]$ in (31), we obtain (29). 
Proof of Theorem 9.1 in the case $l=p$. An $A$-module $M$ will be said to be special if $M=A / A \cdot \lambda$ for some $\lambda$ lying in the centre $\mathbb{Z}_{p}\left[F^{a}\right]$ of $A$, in which case $\lambda=m_{M}\left(F^{a}\right)$ and $P_{M}(t)=m_{M}(t)^{a}$. We let $k$ denote the $A$-module with underlying $W$-module $\mathbb{F}_{q}$ and with $F$ acting as 0 .

Let $\mathcal{R}_{p, f}$ be the full subcategory of $\mathcal{R}_{p}$ of finite objects. Then Exts computed in $\mathcal{R}_{p, f}$ coincide with those in $\mathcal{R}_{p}$ (see the argument on p. 535) and with those in Pro- $\mathcal{R}_{p, f}\left(\mathcal{R}_{p, f}\right.$ is Artinian and so we can apply the opposite of 7.1$)$. There is a canonical functor $\mathcal{R}_{p} \rightarrow$ Pro- $\mathcal{R}_{p, f}$, and by using the usual exact sequences (14) one deduces that Exts in $\mathcal{R}_{p}$ coincide with those in Pro- $\mathcal{R}_{p, f}$. This allows us to use resolutions by free finitely generated $A$-modules to compute Exts in $\mathcal{R}_{p}$.

The case $M$ or $N$ is finite with $F$ acting invertibly. Because of (7), this case is essentially the same as the non- $p$ case. From the spectral sequence (7.15), we obtain exact sequences

$$
0 \rightarrow \operatorname{Ext}_{\mathcal{\mathcal { R }}_{p}}^{i-1}\left(M_{W(\mathbb{F})}, N_{W(\mathbb{F})}\right)_{\Gamma} \rightarrow \operatorname{Ext}_{\mathcal{R}_{p}}^{i}(M, N) \rightarrow \operatorname{Ext}_{\overline{\mathcal{R}}_{p}}^{i}\left(M_{W(\mathbb{F})}, N_{W(\mathbb{F})}\right)^{\Gamma} \rightarrow 0 .
$$

In this case, the groups $\operatorname{Ext}_{\overline{\mathcal{R}}_{p}}^{i}\left(M_{W(\mathbb{F})}, N_{W(\mathbb{F})}\right)$ are finite, and it follows immediately that the alternating product of the orders of the $\operatorname{Ext}_{\mathcal{R}_{p}}^{i}(M, N)$ is 1 .

The case of $M=k$ and $N$ finite. We have the following Koszul-type resolution of $k$ :

$$
0 \rightarrow A \stackrel{t}{\rightarrow} A \oplus A \stackrel{s}{\rightarrow} A \rightarrow k \rightarrow 0
$$

where $t(\alpha)=(\alpha F, p \alpha)$ and $s(x, y)=p x-y F$. For any $N$, the groups $\operatorname{Ext}^{i}(k, N)$ are the cohomology groups of the complex

$$
\operatorname{Hom}(A, N) \stackrel{\circ s}{\longrightarrow} \operatorname{Hom}(A \oplus A, N) \stackrel{\circ t}{\longrightarrow} \operatorname{Hom}(A, N) \rightarrow 0 \rightarrow \cdots .
$$

Therefore, $\operatorname{Ext}^{i}(k, N)=0$ for $i>2$, and if $N$ is finite, then

$$
\operatorname{Ext}^{0}(k, N) \cdot \operatorname{Ext}^{2}(k, N)=\operatorname{Ext}^{1}(k, N)
$$

because the groups in (33) are finite.

The case $M=A / A \lambda$ and $N=k$. The resolution

$$
0 \rightarrow A \stackrel{\cdot \lambda}{\longrightarrow} A \rightarrow M \rightarrow 0
$$

gives an exact sequence

$$
0 \rightarrow \operatorname{Hom}(M, k) \rightarrow \operatorname{Hom}(A, k) \rightarrow \operatorname{Hom}(A, k) \rightarrow \operatorname{Ext}^{1}(M, k) \rightarrow 0
$$

and equalities $\operatorname{Ext}^{i}(M, k)=0(i>1)$. As $\operatorname{Hom}(A, k) \cong k$ is finite, this implies that

$$
\left[\operatorname{Ext}^{0}(M, k)\right]=\left[\operatorname{Ext}^{1}(M, k)\right] \text {. }
$$

The case $M=k$ and $N=A / A \lambda$. From (32) and (33), we find that $\operatorname{Ext}^{i}(k, A)=0$ unless $i=2$ in which case $\operatorname{Ext}^{2}(k, A)=k$. Now the $\operatorname{Ext}(k,-)$-sequence of

$$
0 \rightarrow A \stackrel{\cdot \lambda}{\longrightarrow} A \rightarrow M \rightarrow 0
$$

gives an exact sequence

$$
0 \rightarrow \operatorname{Ext}^{1}(k, N) \rightarrow \operatorname{Ext}^{2}(k, A) \rightarrow \operatorname{Ext}^{2}(k, A) \rightarrow \operatorname{Ext}^{2}(k, N) \rightarrow 0
$$

and equalities $\operatorname{Ext}^{i}(k, N)=0(i \neq 1,2)$. $\operatorname{As}_{\operatorname{Ext}}^{2}(k, A)$ is finite, the sequence gives that

$$
\left[\operatorname{Ext}^{1}(k, N)\right]=\left[\operatorname{Ext}^{2}(k, N)\right] .
$$


The case $M, N$ special, $m_{M}$ and $m_{N}$ relatively prime. Let

$$
\begin{gathered}
M=A / A \lambda_{1}, \quad \lambda_{1}=m_{M}\left(F^{a}\right), \\
N=A / A \lambda_{2}, \quad \lambda_{2}=m_{N}\left(F^{a}\right)
\end{gathered}
$$

with $m_{M}(t)$ and $m_{N}(t)$ relatively prime. From the resolution

$$
0 \rightarrow A \stackrel{\cdot \lambda_{1}}{\longrightarrow} A \rightarrow M \rightarrow 0
$$

we obtain an exact sequence

$$
0 \rightarrow \operatorname{Hom}(M, N) \rightarrow N \stackrel{\lambda_{1} \cdot}{\longrightarrow} N \rightarrow \operatorname{Ext}^{1}(M, N) \rightarrow 0 \rightarrow \ldots
$$

As $\cdot \lambda_{1}$ is injective, we see that $\operatorname{Ext}^{i}(M, N)=0$ for $i \neq 1$, and so

$$
z(f) \cdot\left[\operatorname{Ext}^{2}(M, N)\right]=z\left(\lambda_{1} \cdot \stackrel{9.8}{=}\left|\operatorname{det}\left(\lambda_{1} \cdot\right)\right|_{p}^{a} .\right.
$$

Here $\operatorname{det}\left(\lambda_{1} \cdot\right)$ can be computed as the determinant of $m_{M}\left(F^{a}\right)$ acting on the $B$ vector space $N_{\mathbb{Q}}$. But $P_{M}(t)=m_{M}(t)^{a}$, and so

$$
\operatorname{det}\left(m_{M}\left(F^{a}\right) \mid N_{\mathbb{Q}}\right)^{a}=\prod_{\substack{1 \leq i \leq r(M) \\ 1 \leq j \leq r(N)}}\left(a_{i}-b_{j}\right) .
$$

Thus,

$$
z(f) \cdot\left[\operatorname{Ext}^{2}(M, N)\right]=\left|\prod\left(a_{i}-b_{j}\right)\right|_{p}=\left|q^{s(M) \cdot r(N)} \prod\left(1-\frac{b_{j}}{a_{i}}\right)\right|_{p},
$$

as required.

The case $M$ and $N$ are special and equal. We first give an explicit description of the map

$$
f: \operatorname{Hom}(M, N) \rightarrow \operatorname{Ext}^{1}(M, N)
$$

in the case that $M=A / A \cdot \lambda_{1}$ and $N=A / A \cdot \lambda_{2}$ are special but not necessarily equal. We use ${ }^{-}$to denote $-\otimes_{W\left(\mathbb{F}_{q}\right)} W(\mathbb{F})$, and we let $\sigma_{k}$ be the canonical generator of $\operatorname{Gal}\left(\mathbb{F} / \mathbb{F}_{q}\right)$. The $\operatorname{Ext}(-, N)$ sequence of

$$
0 \rightarrow A \stackrel{\cdot \lambda_{1}}{\rightarrow} A \rightarrow M \rightarrow 0
$$

is

$$
0 \rightarrow \operatorname{Hom}(M, N) \rightarrow N \stackrel{\lambda_{1}}{\rightarrow} N \rightarrow \operatorname{Ext}^{1}(M, N) \rightarrow 0,
$$

and the $\operatorname{Ext}^{r}(-, \bar{N})$ sequence of

$$
0 \rightarrow \bar{A} \stackrel{\lambda_{1}}{\rightarrow} \bar{A} \rightarrow \bar{M} \rightarrow 0
$$

is

$$
0 \rightarrow \operatorname{Hom}(\bar{M}, \bar{N}) \rightarrow \bar{N} \stackrel{\lambda_{1}}{\rightarrow} \bar{N} \rightarrow \operatorname{Ext}^{1}(\bar{M}, \bar{N}) \rightarrow 0 .
$$

The map $f$ can be described as follows: let $u \in \operatorname{Hom}(M, N)$, and regard $u$ as an element of $N$ such that $\lambda_{1} u=0$; then $u$ can be written $u=\left(\sigma_{k}-1\right) v$ for some $v \in \bar{N} ;$ now

$$
\left(\sigma_{k}-1\right)\left(\lambda_{1} v\right)=\lambda_{1}\left(\sigma_{k}-1\right) v=\lambda_{1} u=0,
$$

and so $\lambda_{1} v \in \bar{N}^{\Gamma}=N$; the image of $\lambda_{1} v$ under $N \rightarrow \operatorname{Ext}^{1}(M, N)$ is $f(u)$.

Now take $N=M$, so that multiplication by $\lambda=\lambda_{1}=\lambda_{2}$ is zero on $M$. Thus,

$$
\operatorname{Hom}(M, N)=A / A \lambda=\operatorname{Ext}^{1}(M, N),
$$


and $f$ is an endomorphism of $A / A / \lambda$. Since $A / A \lambda$ is torsion-free, $z(f)$ is defined if and only if

$$
f_{\mathbb{Q}}: A_{\mathbb{Q}} / A_{\mathbb{Q}} \lambda \rightarrow A_{\mathbb{Q}} / A_{\mathbb{Q}} \lambda
$$

has nonzero determinant (9.8), in which case

$$
z(f)=|\operatorname{det}(f)|_{p}^{a} \text {. }
$$

Let $u \in A_{\mathbb{Q}} / A_{\mathbb{Q}} \lambda$, and choose $v \in \bar{A}_{\mathbb{Q}} / \bar{A}_{\mathbb{Q}} \lambda$ such that $u=\sigma_{k} v-v$. Then $\sigma_{k}^{i} v=i u+v$ for all $i$. Let

$$
\lambda(F)=F^{m a}+b_{m a-a} F^{m a-a}+\cdots+b_{0}=m\left(F^{a}\right) .
$$

Then

$$
\begin{aligned}
f(u) & =\lambda(F) v \\
& =m u F^{m a}+(m-1) b_{m a-a} u F^{m a-a}+\cdots+0 \quad(\text { as } v \lambda=0) \\
& =u F^{a} \frac{d}{d F^{a}}\left(m\left(F^{a}\right)\right) \\
& =F^{a} \frac{d}{d F^{a}}\left(m\left(F^{a}\right)\right) u .
\end{aligned}
$$

From (9.8) we find that

$$
z(f)=\left|\operatorname{det}\left(\frac{d}{d F^{a}}\left(m\left(F^{a}\right)\right)\right)\right|_{p}^{a} \cdot\left|\operatorname{det}\left(F^{a}\right)\right|_{p}^{a} .
$$

But

$$
\left|\operatorname{det}\left(F^{a}\right)\right|_{p}^{a}=\left|q^{a \cdot s(N)}\right|_{p}
$$

and

$$
\left|\operatorname{det}\left(\frac{d}{d F^{a}}\left(m\left(F^{a}\right)\right)\right)\right|_{p}^{a}=\left|\prod_{a_{i} \neq b_{j}}\left(a_{i}-b_{j}\right)\right|_{p}=\left|q^{s(N)(r(N)-a)} \prod_{a_{i} \neq b_{j}}\left(1-\frac{b_{j}}{a_{i}}\right)\right|_{p} .
$$

Thus,

$$
z(f)=\left|q^{r(N) \cdot s(N)} \prod_{a_{i} \neq b_{j}}\left(1-\frac{b_{j}}{a_{i}}\right)\right|_{p} .
$$

$\operatorname{As}_{\operatorname{Ext}^{2}}(M, N)=0$, this proves Theorem 9.1 in this case.

The general case. An isogeny (denoted $\sim$ ) of objects in $\mathcal{R}_{p}$ is a homomorphism whose kernel and cokernel have finite length over $W$. We say that an object in $\mathcal{R}_{p}$ is indecomposable if it is not isogenous to a direct sum of two nonzero objects.

Lemma 9.3. Every object of $\mathcal{R}_{p}$ is isogenous to a direct sum of indecomposable objects, and the decomposition is unique up to isogeny.

Proof. Apply the Krull-Schmidt theorem in the category of $A_{\mathbb{Q}}$-modules.

Lemma 9.4. If $M$ in $\mathcal{R}_{p}$ is indecomposable, then $M_{\mathbb{Q}} \approx A_{\mathbb{Q}} / A_{\mathbb{Q}} \lambda$ for some $\lambda \in A_{\mathbb{Q}}$; moreover, the minimum polynomial $m_{M}(t)$ of the Frobenius endomorphism of $M$ is a power of a $\mathbb{Z}_{p}$-irreducible polynomial, and there exists an integer e such that $\oplus^{e} M \sim A / A \cdot m_{M}(t)$. 
Proof. The ring $A_{\mathbb{Q}}$ is the skew polynomial $\operatorname{ring} B[F, \sigma]$. It is therefore a principal ideal domain [25 III, 1, p. 30], and every finitely generated module over such a ring is isomorphic to a direct sum of cyclic modules [25, III, Theorem 19, p. 44]. This proves the first part.

For the second part, we use that the two-sided ideals in $A_{\mathbb{Q}}$ are precisely those generated by a polynomial in the centre $\mathbb{Q}_{p}\left[F^{a}\right]$ of $A_{\mathbb{Q}}[25$. III, 5, p. 38]. It follows that $A_{\mathbb{Q}} \cdot m\left(F^{a}\right)$ is the largest two-sided ideal contained in $A_{\mathbb{Q}} \cdot \lambda$, i.e., it is the bound of the ideal $A_{\mathbb{Q}} \cdot \lambda$ in the sense of [25, III, 6, p. 38]. Because $A_{\mathbb{Q}} / A_{\mathbb{Q}} \lambda$ is indecomposable, this implies that $m(t)$ is a power of a $\mathbb{Z}_{p}$-irreducible polynomial 25, III, Theorem 13, p. 40]. Finally, when $A_{\mathbb{Q}} / A_{\mathbb{Q}} \cdot m\left(F^{a}\right)$ is decomposed into a direct sum of indecomposable modules $A_{\mathbb{Q}} / A_{\mathbb{Q}} \lambda^{\prime}$, each of the ideals $A_{\mathbb{Q}} \lambda^{\prime}$ has $A_{\mathbb{Q}} \cdot m\left(F^{a}\right)$ as its bound and is therefore isomorphic to $M$ [25, III, Theorem 20, p. 45].

Proposition 9.5. Objects of the following types generate $K\left(\mathcal{R}_{p}\right)_{\mathbb{Q}}$ :

(a) $k=\left(\mathbb{F}_{q}, 0\right)$;

(b) $\left(M, F_{M}\right)$ with $M$ finite and $F_{M}$ invertible;

(c) $M$ special and $m_{M}(t)$ is a power of an irreducible polynomial.

Proof. For every finite module $M$, there exists an $n$ such that $M=\operatorname{Im}\left(F^{n}\right) \oplus$ $\operatorname{Ker}\left(F^{n}\right)$ (Fitting's Lemma). It follows that such an $M$ has a composition series with quotients of type (a) or (b). The general statement now follows from Lemma 9.4.

Therefore (see 9.2 f)), it suffices to prove Theorem 9.1 when each of $M$ and $N$ is of type (a), (b), or (c). Moreover, because of the condition on the minimum polynomials, in case (c) we can assume that either $m_{M}$ and $m_{N}$ have no common factor or they are irreducible and equal. This we have done.

Restatement of the theorem for complexes. We sketch a generalization of Theorem 9.1 to complexes. Let $M^{\bullet}$ and $N^{\bullet}$ be bounded complexes of $A$-modules such that $H^{i}\left(M^{\bullet}\right)$ lies in $\mathcal{R}_{p}$ for all $i$. Define

$$
P_{M \bullet \vee \otimes N} \bullet(t)=\prod P_{H^{i}(M \bullet)^{\vee} \otimes H^{j}(N \bullet)}(t)^{(-1)^{i+j}} .
$$

Let $f^{i}: \operatorname{Ext}^{i}\left(M^{\bullet}, N^{\bullet}\right) \rightarrow \operatorname{Ext}^{i+1}\left(M^{\bullet}, N^{\bullet}\right)$ be the map rendering

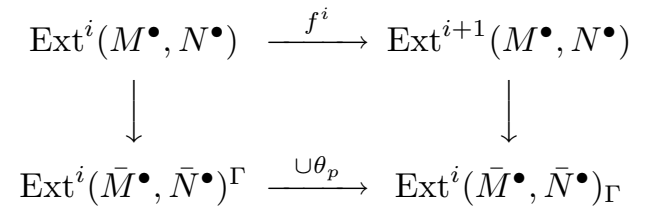

commutative. Here the vertical maps arise from a spectral sequence (cf. 7.15), and the lower map is cup-product with the canonical generator $\theta_{p} \in H^{1}(\Gamma, W)$ (equal to the map induced by the identity map on $\left.\operatorname{Ext}^{i}\left(\bar{M}^{\bullet}, \bar{N}^{\bullet}\right)\right)$. Because $\theta_{p}^{2}=0$,

$$
\operatorname{Ext}^{\bullet}\left(M^{\bullet}, N^{\bullet}\right): \quad \cdots \rightarrow \operatorname{Ext}^{i}\left(M^{\bullet}, N^{\bullet}\right) \stackrel{f^{i}}{\rightarrow} \operatorname{Ext}^{i+1}\left(M^{\bullet}, N^{\bullet}\right) \rightarrow \cdots
$$

is a complex. Define

$$
\chi^{\times}\left(M^{\bullet}, N^{\bullet}\right)=\prod\left[H^{i}\left(\operatorname{Ext}\left(^{\bullet}\left(M^{\bullet}, N^{\bullet}\right)\right)\right]^{(-1)^{i}}\right.
$$

when these numbers are finite. 
Theorem 9.6. Let $M^{\bullet}$ and $N^{\bullet}$ be bounded complexes of $A$-modules such that $H^{i}\left(M^{\bullet}\right)$ and $H^{i}\left(N^{\bullet}\right)$ are semisimple and finitely generated over $W$ for all $i$.

(a) The groups $\operatorname{Ext}^{i}\left(M^{\bullet}, N^{\bullet}\right)$ are finitely generated $\mathbb{Z}_{p}$-modules for all $i$.

(b) The alternating sum $\sum(-1) r_{i}$ of the ranks of the $\operatorname{Ext}^{i}\left(M^{\bullet}, N^{\bullet}\right)$ is zero.

(c) The order of zero of $P\left(M^{\bullet \vee} \otimes N^{\bullet}, t\right)$ at $t=1$ is equal to the secondary Euler characteristic $\sum(-1)^{i+1} \cdot i \cdot r_{i}$.

(d) The groups $H^{i}\left(\operatorname{Ext}^{\bullet}\left(M^{\bullet}, N^{\bullet}\right)\right)$ are finite, and

$$
\begin{gathered}
\left|q^{\chi\left(M^{\bullet}, N^{\bullet}\right)} \cdot \lim _{s \rightarrow 1} \frac{P_{M} \bullet \otimes \otimes N^{\bullet}(t)}{(1-t)^{\rho\left(M^{\bullet}, N^{\bullet}\right)}}\right|_{l}=\chi^{\times}\left(M^{\bullet}, N^{\bullet}\right) \\
\text { where } \chi\left(M^{\bullet}, N^{\bullet}\right)=\sum(-1)^{i+j} s\left(H^{i}\left(M^{\bullet}\right)\right) \cdot r\left(H^{j}\left(N^{\bullet}\right)\right) .
\end{gathered}
$$

Proof. Consider first the case that $M^{\bullet}$ and $N^{\bullet}$ are modules $M$ and $N$ regarded as complexes concentrated in degree 0 .

(a) Because $M$ and $N$ are semisimple, $m(M, t)$ and $m(N, t)$ have only simple roots, and therefore the hypotheses of Theorem 9.1 hold. Hence, the groups $\operatorname{Ext}^{i}(M, N)$ are finitely generated $\mathbb{Z}_{p^{-}}$-modules for all $i$, finite for $i=2$, and zero for $i>2$.

(b) Moreover, $f: \operatorname{Hom}(M, N) \rightarrow \operatorname{Ext}^{1}(M, N)$ has finite kernel and cokernel, and so $\operatorname{Hom}(M, N)$ and $\operatorname{Ext}^{1}(M, N)$ have the same rank.

(c) In fact, one sees easily that the rank of $\operatorname{Hom}(M, N)$ is the number of pairs $(i, j)$ such that $a_{i}=b_{j}$ (see $\left.9.2 \mathrm{~d}\right)$.

(d) The map $f$ of Theorem 9.1 coincides with $f^{0}$, and so (d) is a restatement of the theorem.

The general case now follows from the usual induction argument on the number of nonzero terms in the complexes $M^{\bullet}$ and $N^{\bullet}$ (cf. $9.2 \mathrm{f}$ ).

There is a similar theorem for $l \neq p$, whose formulation and proof we leave to the reader.

Why $\mathcal{R}^{+}\left(\mathbb{F}_{q} ; \mathbb{Z}_{p}\right)$ rather than $\mathcal{R}\left(\mathbb{F}_{q} ; \mathbb{Z}_{p}\right)$ ?

9.7. Write $\mathcal{R}^{+}$and $\mathcal{R}$ for $\mathcal{R}^{+}\left(\mathbb{F}_{q} ; \mathbb{Z}_{p}\right)$ and $\mathcal{R}\left(\mathbb{F}_{q} ; \mathbb{Z}_{p}\right)$, respectively. Even the torsion subgroup of $\operatorname{Ext}_{\mathcal{R}}^{1}(M, N)$ can be infinite. Suppose, for example, that $M$ and $N$ are torsion-free, and consider the diagram

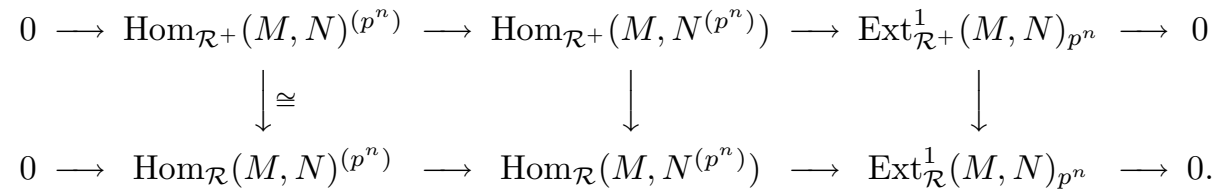

Since $\left[\operatorname{Hom}_{\mathcal{R}}\left(M, N^{\left(p^{n}\right)}\right)\right]=\left(q^{n}\right)^{r(M) r(N)}$ is unbounded, whereas [ $\left.\operatorname{Hom}_{\mathcal{R}^{+}}\left(M, N^{\left(p^{n}\right)}\right)\right]$ is bounded if $\operatorname{Hom}_{R^{+}}(M, N)=0$, we see that $\operatorname{Ext}_{\mathcal{R}}^{1}(M, N)(p)$ is frequently infinite. From a different perspective, it follows from Theorem 9.1 that $\operatorname{Ext}_{\mathcal{R}^{+}}^{1}(M, N)$ grows when $M$ and $N$ are replaced by $M(1)$ and $N(1)$ because $s(M(1))>s(M)$, but the functor $M \mapsto M(1)$ is an equivalence on the category $\mathcal{R}\left(\mathbb{F}_{q} ; \mathbb{Z}_{p}\right)$.

Appendix: Lemmas on abelian groups. Recall that, for a homomorphism $f: M \rightarrow N$ of abelian groups, we define $z(f)=\frac{[\operatorname{Ker}(f)]}{[\operatorname{Coker}(f)]}$ when both the top and bottom are finite. There are the following elementary statements [53, §5]. We let $W=W\left(\mathbb{F}_{q}\right), q=p^{a}$. 
9.8. Let $M$ and $N$ be finitely generated $\mathbb{Z}$-modules (resp. $W$-modules) of the same rank, and let $\left(x_{i}\right)$ and $\left(y_{i}\right)$ be bases for $M$ and $N$, respectively, modulo torsion. Suppose $f\left(x_{i}\right) \equiv \sum a_{i j} y_{j}$ modulo torsion. Then $z(f)$ is defined if and only if $\operatorname{det}\left(a_{i j}\right) \neq 0$, in which case

$$
z(f)=\frac{\left[M_{\text {tors }}\right]}{\left|\operatorname{det}\left(a_{i j}\right)\right|\left[N_{\text {tors }}\right]}
$$

(resp.,

$$
\left.z(f)=\frac{\left[M_{\text {tors }}\right]\left|\operatorname{det}\left(a_{i j}\right)\right|_{p}^{a}}{\left[N_{\text {tors }}\right]}\right) .
$$

Note that, when $M=N$, $\operatorname{det}\left(a_{i j}\right)$ is the determinant of $f_{\mathbb{Q}}: M_{\mathbb{Q}} \rightarrow M_{\mathbb{Q}}$.

9.9. Consider maps $f: M \rightarrow N$ and $g: N \rightarrow P$. If any two of the three numbers $z(f), z(g), z(g \circ f)$ are defined, then so is the third, and

$$
z(g \circ f)=z(g) \cdot z(f) .
$$

9.10. Let $\Gamma=\hat{\mathbb{Z}}$, and let $\gamma$ be its canonical topological generator. Let $M$ be a $\Gamma$ module that is finitely generated as a $\mathbb{Z}$-module, and let $f: M^{\Gamma} \rightarrow M_{\Gamma}$ be the map induced by the identity map on $M$. Then $z(f)$ is defined if and only if the minimum polynomial of $\gamma$ on $M_{\mathbb{Q}}$ does not have 1 as a multiple root, in which case

$$
z(f)\left|\prod_{a_{i} \neq 1}\left(1-a_{i}\right)\right|=1
$$

where $a_{1}, a_{2}, \ldots$ is the family of eigenvalues of $\gamma$ acting on $M_{\mathbb{Q}}$.

\section{Extensions and Zeta Functions: Global CASE}

In this section, $\mathcal{M}(\mathbb{F} ; \mathbb{Q})$ will be one of the following categories:

(a) the category $\mathcal{M}_{\text {num }}(\mathbb{F} ; \mathbb{Q} ; \mathcal{S})$ where $\mathcal{S}$ consists of all smooth projective varieties over $\mathbb{F}$ and we assume that the Tate conjecture holds for all $V \in \mathcal{S}$;

(b) the category $\mathcal{M}(\mathbb{F} ; \mathbb{Q})$ defined in $(5.27)$.

In fact, if the category in (a) exists and the Hodge conjecture holds for complex abelian varieties of CM-type, then these categories are essentially the same: the reduction functor $\mathbf{C M}\left(\mathbb{Q}^{\text {al }}\right) \rightarrow \mathcal{M}_{\text {num }}(\mathbb{F} ; \mathbb{Q} ; \mathcal{S})$ defines a fibre functor $\omega$ on $\mathbf{C M}\left(\mathbb{Q}^{\text {al }}\right)^{P}$, and the quotient of $\mathbf{C M}\left(\mathbb{Q}^{\text {al }}\right)$ corresponding to $\omega$ is canonically equivalent with $\mathcal{M}_{\text {num }}(\mathbb{F} ; \mathbb{Q} ; \mathcal{S})$.

\section{The main theorem for extensions.}

Theorem 10.1. For all effective motives $X$ and $Y$ in $\mathcal{M}^{+}\left(\mathbb{F}_{q} ; \mathbb{Z}\right)$, the groups $\operatorname{Ext}^{1}(X, Y)$ and $\operatorname{Ext}^{2}(X, Y)$ cotors are finite, and

$$
q^{\chi(X, Y)} \cdot \zeta\left(X^{\vee} \otimes Y, s\right) \sim \pm \frac{\left[\operatorname{Ext}^{1}(X, Y)\right] \cdot D(X, Y)}{\left[\operatorname{Hom}(X, Y)_{\text {tors }}\right] \cdot\left[\operatorname{Ext}^{2}(X, Y)_{\text {cotors }}\right]} \cdot\left(1-q^{-s}\right)^{\rho(X, Y)}
$$

as $s \rightarrow 0$ where $\chi(X, Y)=s\left(X_{p}\right) r\left(Y_{p}\right)$ (notations as on $p$. 539), $D=D(X, Y)$ is the discriminant of the pairing

$$
\operatorname{Hom}(Y, X) \times \operatorname{Hom}(X, Y) \stackrel{\circ}{\rightarrow} \operatorname{End}(Y) \stackrel{\text { trace }}{\longrightarrow} \mathbb{Z},
$$

and $\rho(X, Y)$ is the rank of $\operatorname{Hom}(X, Y)$. 
Remark 10.2. Let $X$ and $Y$ be effective motives over $\mathbb{F}_{q}$.

(a) Because $\mathcal{M}^{+}\left(\mathbb{F}_{q} ; \mathbb{Q}\right)$ is a semisimple category, $\operatorname{End}\left(X_{0} \times Y_{0}\right)$ is a semisimple ring. As it is also finite dimensional over $\mathbb{Q}$, the trace pairing

$$
\operatorname{End}\left(X_{0} \times Y_{0}\right) \times \operatorname{End}\left(X_{0} \times Y_{0}\right) \rightarrow \mathbb{Q}
$$

is nondegenerate: $D(X, Y) \neq 0$.

(b) Below we prove that $\left[\operatorname{Ext}^{2}(X, Y)_{\text {cotors }}\right]=\left[\operatorname{Hom}(Y, X)_{\text {tors }}\right]$. This makes the formula more symmetric; note that $D(X, Y)=D(Y, X)$.

(c) When we replace $X$ by $X(r)$ or $Y$ by $Y(r)(r \in \mathbb{N})$ in Theorem 10.1, we obtain a description of the behaviour of $\zeta\left(X^{\vee} \otimes Y, s\right)$ near $-r$ or $+r$.

Proof of Theorem 10.1 with $z(\varepsilon)$ for $D(X, Y)$. Recall (8.1) that for all $l$, there is an exact sequence

$$
0 \rightarrow \operatorname{Ext}_{\mathcal{M}^{+}\left(\mathbb{F}_{q} ; \mathbb{Z}\right)}(X, Y)_{\mathbb{Z}_{l}} \stackrel{c_{l}}{\longrightarrow} \operatorname{Ext}_{\mathcal{R}^{+}\left(\mathbb{F}_{q} ; \mathbb{Z}_{l}\right)}^{1}\left(X_{l}, Y_{l}\right) \rightarrow T_{l} \operatorname{Ext}_{\mathcal{M}^{+}\left(\mathbb{F}_{q} ; \mathbb{Z}_{l}\right)}(X, Y) \rightarrow 0 .
$$

Define

$$
\varepsilon=\left(\varepsilon_{l}\right)_{l}: \operatorname{Hom}_{\mathcal{M}^{+}\left(\mathbb{F}_{q} ; \mathbb{Z}\right)}(X, Y)_{\hat{\mathbb{Z}}} \rightarrow T \operatorname{Ext}_{\mathcal{M}^{+}\left(\mathbb{F}_{q} ; \mathbb{Z}\right)}(X, Y)
$$

to be the map making

$$
\begin{array}{cc}
\operatorname{Hom}_{\mathcal{M}^{+}\left(\mathbb{F}_{q} ; \mathbb{Z}\right)}(X, Y)_{\mathbb{Z}_{l}} \stackrel{\varepsilon_{l}}{\longrightarrow} T_{l} \operatorname{Ext}_{\mathcal{M}^{+}\left(\mathbb{F}_{q} ; \mathbb{Z}\right)}^{2}(X, Y) & \uparrow c_{l} \\
\lfloor & \cong \\
\operatorname{Hom}_{\mathcal{R}^{+}\left(\mathbb{F}_{q} ; \mathbb{Z}_{l}\right)}\left(X_{l}, Y_{l}\right) \stackrel{f_{l}}{\longrightarrow} & \operatorname{Ext}_{\mathcal{R}^{+}\left(\mathbb{F}_{q} ; \mathbb{Z}_{l}\right)}^{1}\left(X_{l}, Y_{l}\right)
\end{array}
$$

commute. Here $f_{l}=f\left(X_{l}, Y_{l}\right)$ is the map defined on p. 539. According to 9.9 and 9.1

$$
z\left(\varepsilon_{l}\right)=z\left(c_{l}\right) \cdot z\left(f_{l}\right)=\frac{\left[\operatorname{Ext}^{1}(X, Y)(l)\right]}{\left[\operatorname{Ext}^{2}\left(X_{l}, Y_{l}\right)\right]} \cdot\left|q^{s\left(X_{l}\right) \cdot r\left(Y_{l}\right)} \cdot \prod_{a_{i} \neq b_{j}}\left(1-\frac{b_{j}}{a_{i}}\right)\right|_{l} .
$$

The $a_{i}, b_{j}$ are algebraic numbers independent of $l$. Because $\operatorname{Ext}^{i}\left(X_{l}, Y_{l}\right)=0$ for $i \geq 3$, 8.1b) shows that $\operatorname{Ext}^{i}(X, Y)=0$ for $i \geq 3$, and it follows (from 8.1b again) that

$$
\operatorname{Ext}^{2}(X, Y)_{\text {cotors }}(l) \cong \operatorname{Ext}^{2}\left(X_{l}, Y_{l}\right) .
$$

On multiplying the equations (35) for the different $l$ and applying the product formula, we find that

$$
q^{\chi(X, Y)} \cdot \prod_{a_{i} \neq b_{j}}\left(1-\frac{b_{j}}{a_{i}}\right) \cdot z(\varepsilon)= \pm \frac{\left[\operatorname{Ext}^{1}(X, Y)\right]}{\left[\operatorname{Ext}^{2}(X, Y)_{\text {cotors }}\right]} .
$$

This will become Theorem 10.1 once we have shown that

$$
z(\varepsilon)= \pm \frac{\left[\operatorname{Hom}(X, Y)_{\text {tors }}\right]}{D(X, Y)} .
$$


Comparison of $z(\varepsilon)$ with $D(X, Y)$. Let $N$ be a finitely generated torsion-free discrete $\Gamma$-module, and let $N^{\vee}=\operatorname{Hom}(N, \mathbb{Z})$. The pairing

$$
N^{\Gamma} \times H^{2}\left(\Gamma, N^{\vee}\right) \rightarrow H^{2}(\Gamma, \mathbb{Z}) \cong \mathbb{Q} / \mathbb{Z}
$$

realizes the compact group $\left(N^{\Gamma}\right)_{\hat{\mathbb{Z}}}$ as the Pontryagin dual of the discrete group $H^{2}\left(\Gamma, N^{\vee}\right)$ (e.g., [39 I, 1.10].) There is therefore a canonical isomorphism

$$
\operatorname{Hom}\left(N^{\Gamma}, \hat{\mathbb{Z}}\right) \rightarrow \operatorname{Hom}\left(\mathbb{Q} / \mathbb{Z}, H^{2}\left(\Gamma, N^{\vee}\right)\right) \stackrel{\mathrm{df}}{=} T H^{2}\left(\Gamma, N^{\vee}\right) .
$$

Suppose we are also given a nondegenerate pairing

$$
\psi: N \times M \rightarrow \mathbb{Z}
$$

where $M$ is a discrete $\Gamma$-module such that $M_{\text {tors }}$ is killed by some integer and $M / M_{\text {tors }}$ is finitely generated. This defines a map $M \rightarrow N^{\vee}$ with torsion kernel and cokernel, which induces isomorphisms $H^{2}(\Gamma, M) \rightarrow H^{2}\left(\Gamma, N^{\vee}\right)$ and $T H^{2}(\Gamma, M) \rightarrow$ $T H^{2}\left(\Gamma, N^{\vee}\right)$. Hence, there is a canonical isomorphism

$$
\operatorname{Hom}\left(N^{\Gamma}, \hat{\mathbb{Z}}\right) \rightarrow T H^{2}(\Gamma, M) .
$$

When combined with the map

$$
M^{\Gamma} \rightarrow \operatorname{Hom}\left(N^{\Gamma}, \mathbb{Z}\right)
$$

defined by $\psi$, this gives a homomorphism

$$
\varepsilon(\psi):\left(M^{\Gamma}\right)_{\hat{\mathbb{Z}}} \rightarrow T H^{2}(\Gamma, M) .
$$

Note that $z(\varepsilon(\psi))$ is defined if and only if $M_{\text {tors }}^{\Gamma}$ is finite and the restriction $\psi^{\Gamma}$ to a pairing $N^{\Gamma} \times M^{\Gamma} \rightarrow \mathbb{Z}$ is nondegenerate, in which case

$$
z(\varepsilon(\psi))= \pm \frac{\left[M_{\text {tors }}^{\Gamma}\right]}{\operatorname{det}\left(\psi^{\Gamma}\right)} .
$$

When we apply this remark to the pairing

$$
\operatorname{Hom}(\bar{Y}, \bar{X}) /\{\text { torsion }\} \times \operatorname{Hom}(\bar{X}, \bar{Y}) \rightarrow \mathbb{Q},
$$

we obtain a homomorphism

$$
\varepsilon: \operatorname{Hom}(X, Y)_{\hat{\mathbb{Z}}} \rightarrow T H^{2}(\Gamma, \operatorname{Hom}(X, Y)),
$$

and

$$
z(\varepsilon)= \pm \frac{\left[\operatorname{Hom}(X, Y)_{\mathrm{tors}}\right]}{D(X, Y)} .
$$

Since $\varepsilon$ coincides with the map defined in the preceding subsubsection, this completes the proof of Theorem 10.1.

Comparison of $\operatorname{Hom}(Y, X)_{\text {tors }}$ with $\operatorname{Ext}^{2}(X, Y)_{\text {cotors. }}$. In this subsection, we prove that

$$
\left[\operatorname{Hom}(Y, X)_{\text {tors }}\right]=\left[\operatorname{Ext}^{2}(X, Y)_{\text {cotors }}\right] .
$$

It follows from Theorem 8.1 that

$$
\operatorname{Hom}(Y, X)(l) \cong \operatorname{Hom}\left(Y_{l}, X_{l}\right)_{\text {tors }}, \quad \operatorname{Ext}^{2}(X, Y)_{\text {cotors }}(l) \cong \operatorname{Ext}^{2}\left(X_{l}, Y_{l}\right)_{\text {cotors }},
$$

and so it suffices to show that, for $M$ and $N$ in $\mathcal{R}^{+}\left(\mathbb{F}_{q} ; \mathbb{Z}_{l}\right)$,

$$
\left[\operatorname{Hom}(N, M)_{\text {tors }}\right]=\left[\operatorname{Ext}^{2}(M, N)_{\text {cotors }}\right] .
$$

Because $\operatorname{Ext}^{3}=0$ in $\mathcal{R}^{+}\left(\mathbb{F}_{q} ; \mathbb{Z}_{l}\right), \operatorname{Ext}^{2}\left(M / M_{\text {tors }}, N\right)$ is divisible and

$$
\operatorname{Ext}^{2}(M, N)_{\text {cotors }} \cong \operatorname{Ext}^{2}\left(M_{\text {tors }}, N\right) \text {. }
$$


As $\operatorname{Hom}\left(N, M_{\text {tors }}\right) \cong \operatorname{Hom}(N, M)_{\text {tors }}$, this shows that it suffices to prove that

$$
[\operatorname{Hom}(N, M)]=\left[\operatorname{Ext}^{2}(M, N)\right]
$$

when $M$ is torsion. In fact, we shall show that

$$
\operatorname{Hom}(N, M) \cong \operatorname{Ext}^{2}(M, N)^{*}
$$

when $N$ is torsion-free and $M$ is torsion. Here $*$ denotes $\operatorname{Hom}(-, \mathbb{Q} / \mathbb{Z})$. This implies (36) because an arbitrary $N$ has a resolution by torsion-free objects $(1.4 \mathrm{~b}$, 7.10).

Define

$$
\operatorname{Ext}^{i}(M, N \otimes \mathbb{Q} / \mathbb{Z})=\underset{n}{\lim } \operatorname{Ext}^{i}\left(M, N^{\left(l^{n}\right)}\right)
$$

Then

$$
\operatorname{Ext}^{1}(M, N \otimes \mathbb{Q} / \mathbb{Z}) \cong \operatorname{Ext}^{2}(M, N),
$$

and so we have to show that

$$
\operatorname{Hom}(N, M) \cong \operatorname{Ext}^{1}(M, N \otimes \mathbb{Q} / \mathbb{Z})^{*}
$$

( $M$ torsion, $N$ torsion-free).

Proof of (38) in the case $l \neq p$. We let $\bar{M}$ and $\bar{N}$ denote $M$ and $N$ regarded as $\mathbb{Z}_{l}$-modules. There is a perfect pairing of finite $\mathbb{Z}_{l}$-modules:

$$
\begin{aligned}
\operatorname{Hom}(\bar{N}, \bar{M}) \times \operatorname{Hom}(\bar{M}, \bar{N} \otimes \mathbb{Q} / \mathbb{Z}) \stackrel{\circ}{\rightarrow} & \operatorname{Hom}(\bar{N}, \bar{N} \otimes \mathbb{Q} / \mathbb{Z}) \\
& \cong \operatorname{End}(\bar{N}) \otimes \mathbb{Q} / \mathbb{Z} \stackrel{\operatorname{Tr} \otimes 1}{\longrightarrow} \mathbb{Q} / \mathbb{Z} .
\end{aligned}
$$

This gives a perfect pairing of $\Gamma$-cohomology groups

$$
\operatorname{Hom}(\bar{N}, \bar{M})^{\Gamma} \times \operatorname{Hom}(\bar{M}, \bar{N} \otimes \mathbb{Q} / \mathbb{Z})_{\Gamma} \rightarrow \mathbb{Q} / \mathbb{Z},
$$

which can be identified with (38).

Proof of (38) in the case $l=p$. If $F$ acts as an isomorphism on $M$, this can be proved as in the case $l \neq p$. Thus, we may assume that $F$ acts nilpotently on $M$, which therefore has a composition series whose quotients are isomorphic to $k={ }_{\text {df }}\left(\mathbb{F}_{q}, 0\right)$.

Let $\mathcal{R}_{p^{n}}$ be the subcategory of $\mathcal{R}^{+}\left(\mathbb{F}_{q} ; \mathbb{Z}_{p}\right)$ of objects killed by $p^{n}$. An argument using an analogue of the diagram (23) shows that

$$
\operatorname{Ext}_{\mathcal{R}^{+}\left(\mathbb{F}_{q} ; \mathbb{Z}_{p}\right)}^{1}\left(N, N^{\left(p^{n}\right)}\right) \cong \operatorname{Ext}_{\mathcal{R}_{p^{n}}}^{1}\left(N^{\left(p^{n}\right)}, N^{\left(p^{n}\right)}\right) .
$$

Any extension of $N^{\left(p^{n}\right)}$ by $N^{\left(p^{n}\right)}$ in $\mathcal{R}_{p^{n}}$ splits as an extension of $W / p^{n} W$-modules, and therefore is described by a $\sigma$-linear endomorphism $\alpha$ of $N^{\left(p^{n}\right)}$. The trace of $\alpha$ acting on $N^{\left(p^{n}\right)}$ is an element of $W / p^{n} W$, and the trace of this element is a well-defined element of $\mathbb{Z}_{p} / p^{n} \mathbb{Z}_{p}$. Thus, we have a homomorphism

$$
\operatorname{Ext}_{\mathcal{R}_{p^{n}}}^{1}\left(N^{\left(p^{n}\right)}, N^{\left(p^{n}\right)}\right) \rightarrow \mathbb{Z}_{p} / p^{n} \mathbb{Z}_{p} \cong p^{-n} \mathbb{Z}_{p} / \mathbb{Z}_{p}
$$

On applying (39) and passing to the direct limit, we obtain a homomorphism

$$
t: \operatorname{Ext}^{1}(N, N \otimes \mathbb{Q} / \mathbb{Z}) \rightarrow \mathbb{Q} / \mathbb{Z} .
$$

We claim that the pairings

$$
\operatorname{Ext}^{i}(N, M) \times \operatorname{Ext}^{1-i}(M, N \otimes \mathbb{Q} / \mathbb{Z}) \rightarrow \operatorname{Ext}^{1}(N, N \otimes \mathbb{Q} / \mathbb{Z}) \stackrel{t}{\rightarrow} \mathbb{Q} / \mathbb{Z}
$$


are perfect for $i \geq 0$. When $N=A / A \cdot \lambda$ and $M=k$, this can be proved directly: either both groups are zero, or the pairing is the trace pairing

$$
\mathbb{F}_{q} \times \mathbb{F}_{q} \rightarrow \mathbb{F}_{p}
$$

Consider an exact sequence

$$
0 \rightarrow N^{\prime} \rightarrow N \rightarrow k \rightarrow 0 .
$$

If the pairings (40) are perfect for $N, k$, and $i \geq 0$, then the diagram

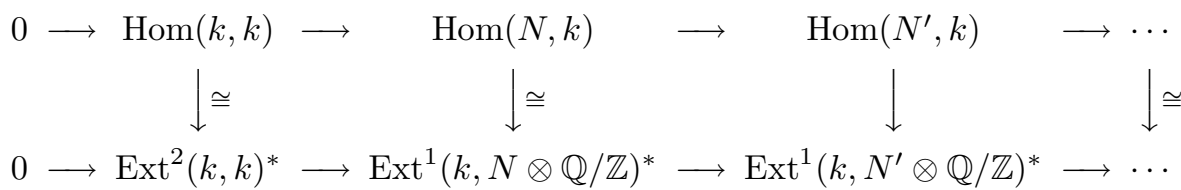

shows that they are perfect for $N^{\prime}, k$, and $i \geq 0$ (note that (24) shows that $\operatorname{Ext}^{i}(k, k)$ equals $k, k \oplus k, k$ respectively for $i=0,1,2)$. A similar argument applies when $N / N^{\prime}$ is a finite module on which $F$ acts invertibly. Since an arbitrary torsion-free $N$ can be realized as a submodule of a direct sum of cyclic modules (Lemma 9.3), this proves that the pairings (40) are perfect when $M=k$. An induction argument on the length of $M$ extends this to an arbitrary torsion $M$. The case $i=0$ completes the proof of (38).

The main theorem for Weil extensions. Let $\Gamma_{0}$ be the subgroup of $\Gamma$ generated by the Frobenius element (so $\Gamma_{0} \cong \mathbb{Z}$ ). Inspired by [35, we define "Weil" extension groups satisfying an analogue of the spectral sequence (1).

As we noted in Proposition 5.20, to give a motive over $\mathbb{F}_{q}$ amounts to giving a motive $X$ over $\mathbb{F}$ together with an endomorphism $\pi$ of $X$ that represents its Frobenius germ. The map $\pi$ can be considered as a descent datum on $X$, and the condition on it a continuity requirement. We now define the category of Weil motives over $\mathbb{F}_{q}, \mathcal{M}\left(\mathbb{F}_{q} ; \mathbb{Z}\right)_{W}$ to be the category of pairs $(X, \pi)$ consisting of a motive $X$ over $\mathbb{F}$ and an endomorphism $\pi$ ("noncontinuous descent datum"). Equivalently, $\mathcal{M}\left(\mathbb{F}_{q} ; \mathbb{Z}\right)_{W}$ is the category of motives over $\mathbb{F}$ with a $\mathbb{Z}$-action. It contains $\mathcal{M}\left(\mathbb{F}_{q} ; \mathbb{Z}\right)$ as a full subcategory.

Similarly, we define the category $\mathcal{M}^{+}\left(\mathbb{F}_{q} ; \mathbb{Z}\right)_{W}$ of effective Weil motives. For effective motives $X$ and $Y$ over $\mathbb{F}_{q}$, let

$$
\operatorname{Ext}^{i}(X, Y)_{0}=\operatorname{Ext}_{\mathcal{M}^{+}\left(\mathbb{F}_{q} ; \mathbb{Z}\right)_{W}}^{i}(X, Y) .
$$

Proposition 10.3. For effective motives $X, Y$ over $\mathbb{F}_{q}$, there is a spectral sequence

$$
H^{i}\left(\Gamma_{0}, \operatorname{Ext}_{\mathcal{M}^{+}(\mathbb{F} ; \mathbb{Z})}^{j}(\bar{X}, \bar{Y})\right) \Longrightarrow \operatorname{Ext}^{i+j}(X, Y)_{0} .
$$

Proof. The proof of Theorem 8.4 can be adapted to the present situation.

Lemma 10.4. The groups $\operatorname{Ext}^{i}(X, Y)_{0}$ are finitely generated for all $i$, torsion for $i \geq 2$, and zero for $i \geq 3$.

Proof. The groups $\operatorname{Ext}_{\mathcal{M}^{+(\mathbb{F} ; \mathbb{Z})}}^{j}(\bar{X}, \bar{Y})$ have composition series whose quotients are finitely generated $\mathbb{Z}$-modules or $\mathbb{F}$ with its natural $\Gamma_{0}$-action. Moreover, they are torsion for $i \geq 1$ and zero for $i \geq 2$. Thus the statement follows from Proposition 10.3. 
Let $f: \operatorname{Hom}(M, N)_{0} \rightarrow \operatorname{Ext}^{1}(M, N)_{0}$ be the map rendering

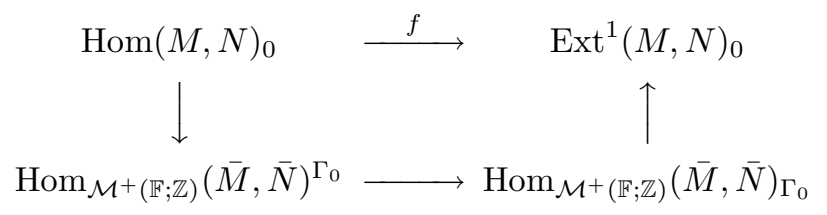

commutative. Here the vertical maps arise from a spectral sequence (10.3) and the lower map is induced by the identity map on $\operatorname{Hom}(\bar{M}, \bar{N})$ (equal to cup-product with the canonical generator of $\left.H^{1}\left(\Gamma_{0}, \mathbb{Z}\right) \cong \mathbb{Z}\right)$.

Theorem 10.5. For effective motives $X$ and $Y$ over $\mathbb{F}_{q}, z(f)$ is defined and

$$
q^{\chi(X, Y)} \cdot \zeta\left(X^{\vee} \otimes Y, s\right) \sim \pm z(f) \cdot\left[\operatorname{Ext}^{2}(X, Y)_{0}\right] \cdot\left(1-q^{-s}\right)^{\rho(X, Y)} \quad \text { as } s \rightarrow 0
$$

(notations as in Theorem 10.1).

Proof. If either $X$ or $Y$ is finite, then the spectral sequence in Proposition 10.3 coincides with that in Theorem 8.4, and so

$$
\operatorname{Ext}^{i}(X, Y) \cong \operatorname{Ext}^{i}(X, Y)_{0} .
$$

Now the same argument as in $\S 8$ proves that, for arbitrary $X, Y$, there are exact sequences

$$
0 \rightarrow \operatorname{Ext}^{i}(X, Y)_{0} \otimes \mathbb{Z}_{l} \rightarrow \operatorname{Ext}^{i}\left(X_{l}, Y_{l}\right) \rightarrow T_{l} \operatorname{Ext}^{i+1}(X, Y)_{0} \rightarrow 0 .
$$

Lemma 10.4 shows that $\operatorname{Ext}^{i+1}(X, Y)_{0}$ has no $l$-divisible elements, and so these sequences reduce to isomorphisms

$$
\operatorname{Ext}^{i}(X, Y)_{0} \otimes \mathbb{Z}_{l} \cong \operatorname{Ext}^{i}\left(X_{l}, Y_{l}\right) .
$$

The spectral sequence (10.3) is compatible with the spectral sequences (7.11) and (7.15), and so the diagrams

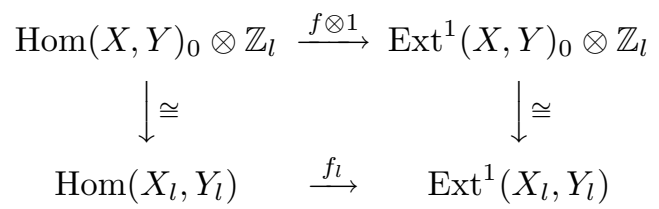

commute. Here $f_{l}$ is the map in Theorem 9.1. Therefore, Theorem 10.5 follows from Theorem 9.1 and the product formula.

Remark 10.6. The advantage of statement 10.5 over 10.1 is that it has a natural extension to complexes of motives; see the sequel to this paper. Compare also Theorem 9.6.

Motivic cohomology. Let $V$ be a smooth projective variety 14 over $\mathbb{F}_{q}$. In contrast to the pure case, $V$ should define, not a mixed motive, but rather a complex which will not (in general) decompose when there is torsion, the torsion at $p$ being particularly complicated. Nevertheless, for an $r \geq 0$ such that the truncated de Rham-Witt cohomology groups $H^{i}\left(V, W \Omega^{\leq r-1}\right)$ are finitely generated over $W$, we make the ad hoc definition: $h^{i} V(r)$ is the isomotive $h_{0}^{i} V(r)$ endowed with the $\mathbb{Z}$-structure provided by the maps $H^{i}\left(V, \mathbb{Z}_{l}(r)\right) \rightarrow H^{i}\left(V, \mathbb{Q}_{l}(r)\right.$ ) (étale cohomology

\footnotetext{
${ }^{14}$ If the category $\mathcal{M}(\mathbb{F} ; \mathbb{Q})$ at the start of this section is taken to be as in 5.27 (case (b)), then $V$ must be taken to be an abelian variety.
} 
for $l \neq p$ and crystalline cohomology $l=p$ ). Define the (Weil) motivic cohomology groups by 15

$$
H_{\text {mot }}^{j}(V, \mathbb{Z}(r))=\bigoplus_{i} \operatorname{Ext}^{i}\left(\mathbb{1}, h^{j-i} V(r)\right)_{0}
$$

Let

$$
H_{\text {mot }}^{\bullet}(V, \mathbb{Z}(r))=\cdots \rightarrow H_{\text {mot }}^{j}(V, \mathbb{Z}(r)) \stackrel{f^{j}}{\longrightarrow} H_{\text {mot }}^{j+1}(V, \mathbb{Z}(r)) \rightarrow \cdots
$$

be the complex with

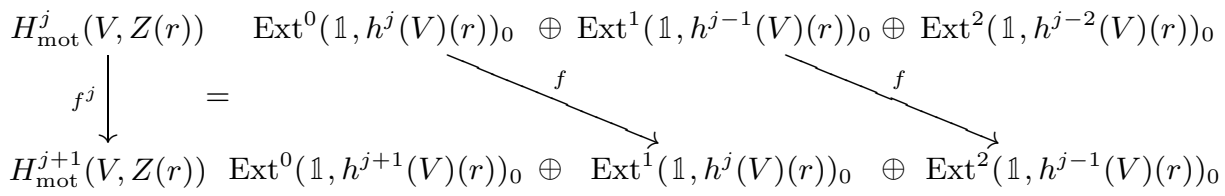

The maps $f$ at right are as in Theorem 10.5 ,

Theorem 10.7. Let $V$ be a smooth projective variety over $\mathbb{F}_{q}$, and let $r \geq 0$ be such that the groups $H^{i}\left(V, W \Omega^{\leq r-1}\right)$ are finitely generated over $W$.

(a) The groups $H_{\text {mot }}^{i}(V, \mathbb{Z}(r))$ are finitely generated abelian groups.

(b) The alternating sum $\sum(-1)^{i} r_{i}$ of the ranks $r_{i}$ of the groups $H_{\text {mot }}^{i}(V, \mathbb{Z}(r))$ is zero.

(c) The order of the zero of $\zeta(V, s)$ at $s=r$ is equal to the secondary Euler characteristic $\rho(V, r)=_{\mathrm{df}} \sum(-1)^{i} i r_{i}$.

(d) The cohomology groups of the complex $\left(H_{\text {mot }}^{\bullet}(V, \mathbb{Z}(r)), f\right)$ are finite, and the alternating product of their orders $\chi^{\times}(V, \mathbb{Z}(r))$ satisfies

$$
\zeta(V, s) \sim \pm \chi^{\times}(V, \mathbb{Z}(r)) \cdot q^{\chi(V, \mathcal{O}, r)} \cdot\left(1-q^{r-s}\right)^{\rho} \text { as } s \rightarrow r
$$

where

$$
\chi(V, \mathcal{O}, r)=\sum_{\substack{0 \leq i \leq r \\ 0 \leq j \leq \operatorname{dim} V}}(-1)^{i+j}(r-i) \cdot \operatorname{dim}_{\mathbb{F}_{q}} H^{j}\left(X, \Omega^{i}\right) .
$$

Proof. Immediate consequence of Theorem[10.5 and [38, 4.1] (the condition on the de Rham-Witt groups implies that the numbers $d^{i}(r-1)$ in the second reference are zero).

Remark 10.8. When $r=0$, the condition on the de Rham-Witt groups is satisfied vacuously, and the groups $H_{\text {mot }}^{i}(V, \mathbb{Z}(0))$ coincide with the Weil-étale groups $H_{W}^{i}(V, \mathbb{Z})$ of Lichtenbaum [35]. Thus, in this case the theorem coincides with the smooth projective case of [35, Theorem 8.2].

\footnotetext{
${ }^{15}$ According to [9] 3.2.1], the motivic cohomology groups ( $\mathbb{Q}$-coefficients) should be the final term of a spectral sequence

$$
E_{2}^{i j}=\operatorname{Ext}^{i}\left(\mathbb{1}, h^{j}(V)\right) \Longrightarrow H_{\text {mot }}^{i+j}(V, \mathbb{Q}(r)) .
$$

Over $\mathbb{F}_{q}$ it is natural to expect this also with $\mathbb{Z}$-coefficients and (following Lichtenbaum's ideas) replace the Exts with the Weil Exts:

$$
E_{2}^{i j}=\operatorname{Ext}^{i}\left(\mathbb{1}, h^{j}(V)\right)_{0} \Longrightarrow H_{\mathrm{mot}}^{i+j}(V, \mathbb{Z}(r))
$$

If this spectral sequence degenerates, then we arrive at (41). In a sequel to this paper, we plan to take a less ad hoc approach.
} 


\section{ACKNOWLEDGEMENTS}

We thank the referee for his careful reading of the manuscript, which saved it from many obscurities.

\section{REFERENCES}

[1] André, Yves, Pour une théorie inconditionnelle des motifs. Inst. Hautes Études Sci. Publ. Math. No. 83, (1996), 5-49. MR 98m:14022

[2] André, Yves; Kahn, Bruno, Construction inconditionnelle de groupes de Galois motiviques. C. R. Math. Acad. Sci. Paris 334 (2002), no. 11, 989-994. MR 2003k:14020

[3] Atiyah, M. F., and Hirzebruch, F., Analytic cycles on complex manifolds. Topology 1 (1962), 25-45. MR 26:3091

[4] Ballico, E., Ciliberto, C., and Catanese F., Trento examples. In: Classification of irregular varieties. Minimal models and abelian varieties. Proceedings of the conference held in Trento, December 17-21, 1990. Edited by E. Ballico, F. Catanese and C. Ciliberto. Lecture Notes in Mathematics, 1515. Springer-Verlag, Berlin, 1992, 134-139. MR 93d:14005

[5] Bloch, Spencer, and Esnault, Hélène, The coniveau filtration and non-divisibility for algebraic cycles. Math. Ann. 304 (1996), no. 2, 303-314. MR 97c:14003

[6] Brosnan, Patrick, Steenrod operations in Chow theory. Trans. Amer. Math. Soc. 355 (2003), no. 5, 1869-1903.

[7] Deligne, P., Le groupe fondamental de la droite projective moins trois points. Galois groups over $\mathbb{Q}$ (Berkeley, CA, 1987), 79-297, Math. Sci. Res. Inst. Publ., 16, Springer, New YorkBerlin, 1989. MR 90m:14016

[8] Deligne, P., Catégories tannakiennes. The Grothendieck Festschrift, Vol. II, 111-195, Progr. Math., 87, Birkhäuser Boston, Boston, MA, 1990. MR 92d:14002

[9] Deligne, Pierre, À quoi servent les motifs? Motives (Seattle, WA, 1991), 143-161, Proc. Sympos. Pure Math., 55, Part 1, Amer. Math. Soc., Providence, RI, 1994. MR 95c:14013

[10] Deligne, Pierre, and Milne, James, Tannakian Categories. In: Hodge cycles, motives, and Shimura varieties. Lecture Notes in Mathematics, 900. Springer-Verlag, Berlin-New York, 1982, 101-228. MR 84m:14046

[11] Demazure, Michel, Lectures on p-divisible groups. Lecture Notes in Mathematics, 302. Springer-Verlag, Berlin-New York, 1972. MR 49:9000

[12] Dwyer, William G., and Friedlander, Eric M., Algebraic and étale $K$-theory. Trans. Amer. Math. Soc. 292 (1985), no. 1, 247-280. MR 87h:18013

[13] Ekedahl, Torsten, Diagonal complexes and $F$-gauge structures. Travaux en Cours. Hermann, Paris, 1986. MR 88g:14015

[14] Faltings, Gerd, and Wüstholz, Gisbert (Eds). Rational points. Papers from the seminar held at the Max-Planck-Institut für Mathematik, Bonn, 1983/1984. Aspects of Mathematics, E6. Friedr. Vieweg \& Sohn, Braunschweig; distributed by Heyden \& Son, Inc., Philadelphia, Pa., 1984. MR 87h:14016

[15] Fontaine, Jean-Marc, and Mazur, Barry, Geometric Galois representations. Elliptic curves, modular forms, and Fermat's last theorem (Hong Kong, 1993), 41-78, Ser. Number Theory, I, Internat. Press, Cambridge, MA, 1995. MR 96h:11049

[16] Fontaine, J.-M., and Illusie, L., p-adic periods: a survey. Proceedings of the Indo-French Conference on Geometry (Bombay, 1989), 57-93, Hindustan Book Agency, Delhi, 1993. MR 95e:14013

[17] Gabber, Ofer, Sur la torsion dans la cohomologie $l$-adique d'une variété. C. R. Acad. Sci. Paris Sér. I Math. 297 (1983), no. 3, 179-182. MR 85f:14018

[18] Giraud, Jean. Méthode de la descente. Bull. Soc. Math. France Mém. 2 (1964), viii+150 pp. MR 32:7556

[19] Giraud, Jean, Cohomologie non abélienne, Springer-Verlag, Berlin, 1971. MR 49:8992

[20] Griffiths, Phillip, and Harris, Joe, On the Noether-Lefschetz theorem and some remarks on codimension-two cycles. Math. Ann. 271 (1985), no. 1, 31-51. MR 87a:14030

[21] Hanamura, Masaki, Mixed motives and algebraic cycles. I. Math. Res. Lett. 2 (1995), no. 6, 811-821. MR 97d:14014

[22] Hanamura, Masaki, Mixed motives and algebraic cycles. III. Math. Res. Lett. 6 (1999), no. 1, 61-82. MR 2000d:14011 
[23] Hiller, Howard L., $\lambda$-rings and algebraic $K$-theory. J. Pure Appl. Algebra 20 (1981), no. 3, 241-266. MR 82e:18016

[24] Huber, Annette, Calculation of derived functors via Ind-categories. J. Pure Appl. Algebra 90 (1993), no. 1, 39-48. MR 94i:18001

[25] Jacobson, Nathan, The Theory of Rings. American Mathematical Society Mathematical Surveys, vol. I. American Mathematical Society, New York, 1943. MR 5:31f

[26] Jannsen, Uwe, Motives, numerical equivalence, and semi-simplicity. Invent. Math. 107 (1992), 447-452. MR 93g:14009

[27] Jensen, C. U. Les foncteurs dérivés de lim et leurs applications en théorie des modules. Lecture Notes in Mathematics, Vol. 254. Springer-Verlag, Berlin-New York, 1972. MR 53:10874

[28] de Jong, A. J., Smoothness, semi-stability and alterations. Inst. Hautes Études Sci. Publ. Math. No. 83, (1996), 51-93. MR 98e:14011

[29] de Jong, A. J., Homomorphisms of Barsotti-Tate groups and crystals in positive characteristic. Invent. Math. 134 (1998), no. 2, 301-333, Erratum ibid. 138 (1999), no. 1, 225. MR 2000f:14070a MR 2000f:14070b

[30] Katz, Nicholas M. and Messing, William, Some consequences of the Riemann hypothesis for varieties over finite fields. Invent. Math. 23 (1974), 73-77. MR 48:11117

[31] Kleiman, Steven L., The standard conjectures. Motives (Seattle, WA, 1991), 3-20, Proc. Sympos. Pure Math., 55, Part 1, Amer. Math. Soc., Providence, RI, 1994. MR 95k:14010

[32] Kratzer, Ch., $\lambda$-structure en $K$-théorie algébrique. Comment. Math. Helv. 55 (1980), no. 2, 233-254. MR 81m:18011

[33] Lang, Serge, and Tate, John, Principal homogeneous spaces over abelian varieties. Amer. J. Math. 80 (1958), 659-684. MR 21:4960

[34] Levine, Marc, Mixed motives. Mathematical Surveys and Monographs, 57. American Mathematical Society, Providence, RI, 1998. MR 99i:14025

[35] Lichtenbaum, Stephen, The Weil-étale topology. Preprint (2002) (available at www.math. brown.edu/ slicht/) Compositio Math. (to appear).

[36] Milne, J. S., Extensions of abelian varieties defined over a finite field. Invent. Math. 5 (1968), 63-84. MR 37:5226

[37] Milne, J. S., Étale cohomology. Princeton Mathematical Series, 33. Princeton University Press, Princeton, NJ, 1980. MR 81j:14002

[38] Milne, J. S., Values of zeta functions of varieties over finite fields. Amer. J. Math. 108 (1986), no. 2, 297-360. MR 87g:14019

[39] Milne, J. S., Arithmetic duality theorems. Perspectives in Mathematics, 1. Academic Press, Inc., Boston, Mass., 1986. MR 88e:14028

[40] Milne, J. S., Motives over finite fields. Motives (Seattle, WA, 1991), 401-459, Proc. Sympos. Pure Math., 55, Part 1, Amer. Math. Soc., Providence, RI, 1994. MR 95g:11053

[41] Milne, J. S., Lefschetz motives and the Tate conjecture, Compositio Math. 117 (1999), 47-81. MR 2000i: 11090

[42] Milne, J. S., Gerbes and abelian motives, preprint 2003, arXiv:math.AG/0301304.

[43] Mitchell, Barry, Theory of categories. Pure and Applied Mathematics, Vol. XVII Academic Press, New York-London, 1965. MR 34:2647

[44] Oort, F., Yoneda extensions in abelian categories. Math. Ann. 153 (1964), 227-235. MR 29:140

[45] Quillen, Daniel, On the cohomology and $K$-theory of the general linear groups over a finite field. Ann. of Math. (2) 96 (1972), 552-586. MR 47:3565

[46] Roos, Jan-Erik. Bidualité et structure des foncteurs dérivés de lim dans la catégorie des modules sur un anneau régulier. C. R. Acad. Sci. Paris 254 (1962), $\overleftarrow{1720}-1722$. MR 25:106b

[47] Saavedra Rivano, Neantro, Catégories Tannakiennes. Lecture Notes in Mathematics, Vol. 265. Springer-Verlag, Berlin-New York, 1972. MR 49:2769

[48] Schoen, Chad, An integral analog of the Tate conjecture for one-dimensional cycles on varieties over finite fields. Math. Ann. 311 (1998), no. 3, 493-500. MR 99e:14005

[49] Serre, Jean-Pierre, Groupes de Grothendieck des schémas en groupes réductifs déployés. Inst. Hautes Études Sci. Publ. Math. No. 34 (1968), 37-52. MR 38:159

[50] Soulé, Christophe, $K$-théorie des anneaux d'entiers de corps de nombres et cohomologie étale. Invent. Math. 55 (1979), no. 3, 251-295. MR 81i:12016

[51] Soulé, Christophe, $K$-theory and values of zeta functions. Algebraic $K$-theory and its applications (Trieste, 1997), 255-283, World Sci. Publishing, River Edge, NJ, 1999. MR 2000g:11063 
[52] Tate, John, Endomorphisms of abelian varieties over finite fields. Invent. Math. 2 (1966), 134-144. MR 34:5829

[53] Tate, John, On the conjectures of Birch and Swinnerton-Dyer and a geometric analog, Séminaire Bourbaki: Vol. 1965/66, Exposé 306, 1966. MR 99f:00041

[54] Tate, John, Relations between $K_{2}$ and Galois cohomology. Invent. Math. 36 (1976), 257-274. MR 55:2847

[55] Tate, John, Conjectures on algebraic cycles in $\ell$-adic cohomology. Motives (Seattle, WA, 1991), 71-83, Proc. Sympos. Pure Math., 55, Part 1, Amer. Math. Soc., Providence, RI, 1994. MR 95a:14010

[56] Verdier, Jean-Louis, Des catégories dérivées des catégories abéliennes. Astérisque No. 239 , 1996. MR 98c:18007

[57] Voevodsky, V., Triangulated categories and motives over a field, in Cycles, transfers, and motivic homology theories. Annals of Mathematics Studies, 143. Princeton University Press, Princeton, NJ, 2000, 188-238. MR 2001d:14026

[58] Zarhin, Ju. G., Endomorphisms of Abelian varieties over fields of finite characteristic. (Russian) Izv. Akad. Nauk SSSR Ser. Mat. 39 (1975), no. 2, 272-277, 471. MR 51:8114

2679 Bedford Road, Ann Arbor, Michigan 48104

E-mail address: math@jmilne.org

$U R L$ : www.jmilne.org/math/

Department of Mathematics, University of Maryland, College Park, Maryland 20742

E-mail address: atma@math.umd.edu

$U R L$ : www. math.umd.edu/ atma 